\title{
Computing Eigenspaces with Specified Eigenvalues of a Regular Matrix Pair $(A, B)$ and Condition Estimation: Theory, Algorithms and Software
}

\author{
Bo Kågström and Peter Poromaa* \\ April, 1994 \\ Revised September, 1994 \\ UMINF-94.04,ISSN-0348-0542 \\ (Also published as LAPACK Working Note xx)
}

\begin{abstract}
Theory, algorithms and LAPACK-style software for computing a pair of deflating subspaces with specified eigenvalues of a regular matrix pair $(A, B)$ and error bounds for computed quantities (eigenvalues and eigenspaces) are presented. The reordering of specified eigenvalues is performed with a direct orthogonal transformation method with guaranteed numerical stability. Each swap of two adjacent diagonal blocks in the real generalized Schur form, where at least one of them corresponds to a complex conjugate pair of eigenvalues, involves solving a generalized Sylvester equation and the construction of two orthogonal transformation matrices from certain eigenspaces associated with the diagonal blocks. The swapping of two $1 \times 1$ blocks is performed using orthogonal (unitary) Givens rotations. The error bounds are based on estimates of condition numbers for eigenvalues and eigenspaces. The software computes reciprocal values of a condition number for an individual eigenvalue (or a cluster of eigenvalues), a condition number for an eigenvector (or eigenspace), and spectral projectors onto a selected cluster. By computing reciprocal values we avoid overflow. Changes in eigenvectors and eigenspaces are measured by their change in angle. The condition numbers yield both asymptotic and global error bounds. The asymptotic bounds are only accurate for small perturbations $(E, F)$ of $(A, B)$, while the global bounds work for all $\|(E, F)\|$ up to a certain bound, whose size is determined by the conditioning of the problem. It is also shown how these upper bounds can be estimated. Fortran 77 software that implements our algorithms for reordering eigenvalues, computing (left and right) deflating subspaces with specified eigenvalues and condition number estimation are presented. Computational experiments that illustrate the accuracy, efficiency and reliability of our software are also described.
\end{abstract}

Key words. Regular matrix pair (pencil), generalized Schur form, direct reordering of eigenvalues, generalized Sylvester equation, eigenvectors, deflating subspaces, condition estimation, error bounds, blocked algorithms, LAPACK-style software.

AMS(MOS) subject classifications. Primary 65F15, 65F 05, 65F35, 65 Y 20.

\footnotetext{
*Department of Computing Science, Umeå University, S-901 87 Umeå, Sweden, Electronic addresses: bokgecs.umu.se and peterpocs.umu.se
} 


\section{Contents}

1 Introduction 4

1.1 Notation . . . . . . . . . . . . . . . . . 5

2 Direct Method for Reordering Eigenvalues in the Generalized Real Schur $\begin{array}{ll}\text { Form } & 6\end{array}$

3 Direct Reordering Algorithms with Guaranteed Backward Stability 9

3.1 Justification for Rejecting a Swap . . . . . . . . . . . . . . . 11

3.2 Algorithm Variants for the Stability Tests . . . . . . . . . . . . . 11

4 Condition Numbers and Error Bounds for Eigenvalues and Eigenspaces of a Regular $(A, B) \quad \mathbf{1 3}$

4.1 A Condition Number and Error Bounds for Simple Eigenvalues . . . . . . . 14

4.2 Conditioning and Error Bounds for Left and Right Deflating Subspaces Associated with a Cluster of Eigenvalues . . . . . . . . . . . 16

4.2.1 Block-diagonalization and Separation of Two Matrix Pairs . . . . . 17

4.2.2 Conditioning of Left and Right Deflating Subspaces . . . . . . . . 18

4.2.3 Upper Bound on Perturbations and Global Error Bounds for Deflating Subspaces and Clustered Eigenvalues . . . . . . . . . . 19

4.2.4 Optimal Backward Perturbation of Approximate Left and Right Deflating Subspaces . . . . . . . . . . . . . . 22

4.2.5 Residual-based Error Bound for Approximate Left and Right Deflating Subspaces ........................ 22

4.3 Summary of Error Bounds for Eigenvalues and Eigenspaces . . . . . . . 23

4.4 Condition Estimates and Error Bounds Computed . . . . . . . . . . . . 24

4.4.1 Estimating $\operatorname{Dif}_{u}$ and $\operatorname{Dif}_{l} \ldots \ldots \ldots \ldots \ldots \ldots$

4.4.2 Estimating Dif ${ }_{l}$ for Individual Eigenvectors Associated with a Complex Conjugate Pair of Eigenvalues . . . . . . . . . . . 27

5 Outline of the Software $\quad 28$

5.1 Reordering of Diagonal Blocks . . . . . . . . . . . . . . . 29

5.2 Computing Deflating Subspaces with Specified Eigenvalues . . . . . . . 29

5.3 Condition Estimation and Approximate Error Bounds . . . . . . . . . 30

6 Computational Experiments $\quad 30$

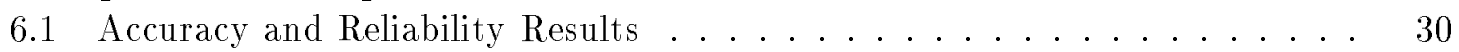

6.1.1 Test Problems . . . . . . . . . . . . . 31

6.1.2 Comparing Different Reordering Methods . . . . . . . . . . 33

6.1.3 Results from Condition Estimation and Error Bounds . . . . . . 36

6.2 A Summary of the Results from the Test Programs . . . . . . . . . . . . 36

6.2 .1 Test problems for $\_\mathrm{CHK} 3 \ldots \ldots \ldots \ldots \ldots$

6.2 .2 A Summary of the Results from _CHK3 . . . . . . . . . . 38

6.2.3 Test Problems and a Summary of the Results from _CHK4 . . . . . 39 
7 Some Conclusions $\quad 40$

$\begin{array}{ll}\text { A Calling sequence DTGEXC } & 49\end{array}$

B Calling sequence DTGEX2 $\quad 51$

C Calling sequence DTGSEN

$\begin{array}{ll}\text { D Calling sequence DTGSNA } & 58\end{array}$

$\begin{array}{lll}\text { E Calling sequence DGSRBB } & 61\end{array}$

\section{List of Tables}

4.1 Asymptotic error bounds for the generalized eigenvalue problem . . . . . . 23

4.2 Global error bounds for the generalized eigenvalue problem . . . . . . . . 24

6.1 Problem characteristics, chordal distances and reciprocal condition numbers 32

6.2 Eigenvalues after reordering for problems 1,6 and $11 \ldots \ldots 34$

6.3 Eigenvalues after reordering for problems 5,10 and $15 \ldots \ldots \ldots$

6.4 Eigenvalues after reordering for problem $12 \ldots \ldots \ldots \ldots \ldots$

7.1 Computed errors after the reordering using Method $1 \ldots \ldots$. . . . . 43

7.2 Computed errors after the reordering using Method $2 \ldots \ldots$. . . . . 44

7.3 Method 2 - Computed stability test values and tolerances . . . . . . . 45

7.4 Computed errors after the reordering using Method 3 . . . . . . . . 46

7.5 Computed errors after the reordering using Algorithm $590 \ldots \ldots$. . . . . 47

7.6 Method 2 - Some computed quantities before and after the reordering . . . 48 


\section{Introduction}

Given a matrix pair ( $A, B$ ), where $A$ and $B$ are general $n \times n$ matrices with real or complex entries. In the generalized eigenvalue problem (GEP) we are interested to find a few or all eigenvalues $\lambda_{i}$ and eigenvectors $x_{i} \neq 0$ such that $A x_{i}=\lambda_{i} B x_{i}$. Mathematically, the eigenvalues are the roots of the characteristic equation $\operatorname{det}(A-\lambda B)=0$. If $B=I_{n}$, GEP reduces to the standard eigenvalue problem (SEP). Moreover, if $\operatorname{det}(B) \neq 0$, the problem can in theory be transformed to $C x=\lambda x$, with $C=B^{-1} A$. If $\operatorname{det}(B)=0$ and $x \neq 0$ is a null vector of $B$, then $B x=0 A x$, i.e. $x$ is an eigenvector of the reciprocal problem $B x=\mu A x$, with $\mu=\lambda^{-1}=0$. In other words, $\lambda=\infty$ is an eigenvalue of $A x=\lambda B x$. By restricting the matrix pencil $A-\lambda B$ (a family of matrices parameterized by $\lambda$ ) such that $\operatorname{det}(A-\lambda B)=0$ if and only if $\lambda$ is an eigenvalue, $(A, B)$ is a regular matrix pair, or similarly, $A-\lambda B$ is a regular matrix pencil. If $\operatorname{det}(A-\lambda B) \equiv 0$ for all $\lambda, A-\lambda B$ is a singular pencil and $(A, B)$ is a singular matrix pair [12].

From a computational point of view it is more natural to define GEP in cross-product form $\beta A x=\alpha B x$ with $\lambda=\alpha / \beta$. An eigenvalue is represented as a pair $(\alpha, \beta)$, where a finite eigenvalue has $\beta \neq 0$ and an infinite eigenvalue has $\beta=0$. In this representation an infinite eigenvalue $(\alpha, 0)$ is not essentially different from a zero eigenvalue $(0, \beta)$. As in SEP we both have right and left eigenvectors $x \neq 0$ and $y \neq 0$, respectively, defined as

$$
\beta A x=\alpha B x, \quad \beta y^{H} A=\alpha y^{H} B .
$$

In several applications (e.g., in control theory [22], [29], [20]) it is not necessary to compute the eigenvectors, but merely to find eigenspaces associated with a specified set of eigenvalues. $\mathcal{L}$ and $\mathcal{R}$ are a pair (left and right) of deflating subspaces of a regular $A-\lambda B$, if $\mathcal{L}=A \mathcal{R}+B \mathcal{R}$ and $\operatorname{dim} \mathcal{L}=\operatorname{dim} \mathcal{R}[25,26]$. One way of computing a pair of deflating subspaces (with orthogonal bases) is via the generalized Schur decomposition. As in the standard eigenvalue problem we distinguish two cases.

The complex case: let $A$ and $B$ be $n \times n$ with complex entries. Then there exist unitary $U \in \mathbf{C}^{n \times n}$ and $V \in \mathbf{C}^{n \times n}$ :

$$
U^{H}(A-\lambda B) V=S-\lambda T,
$$

where $S$ and $T$ are upper triangular. The eigenvalues are given by the pairs $\left(s_{i i}, t_{i i}\right) \neq(0,0)$. The finite eigenvalues are $s_{i i} / t_{i i}$, where $t_{i i} \neq 0$. If $\left(s_{i i}, t_{i i}\right)=(0,0)$ for some $i$, then $(A, B)$ is singular.

The real case: let $A$ and $B$ be $n \times n$ with real entries. Then there exist orthogonal $U \in \mathbf{R}^{n \times n}$ and $V \in \mathbf{R}^{n \times n}$ :

$$
U^{T}(A-\lambda B) V=S-\lambda T,
$$

where $S$ is upper quasi-triangular and $T$ is upper triangular. A quasi-triangular matrix is block upper triangular with $1 \times 1$ or $2 \times 2$ blocks on the diagonal. The $2 \times 2$ blocks on the diagonal of $S-\lambda T$ correspond to pairs of complex conjugate eigenvalues.

The columns of $U$ and $V, u_{i}$ and $v_{i}, i=1: n$, are left and right generalized Schur vectors and the first $k$ columns of $U$ and $V$ span a $k$-dimensional pair of deflating subspaces associated with the $k \times k(1,1)$-block of $(S, T)$ in generalized Schur form. $U$ and $V$ can be chosen so that the eigenvalues appear in any order along the (block) diagonals of $S$ and $T$. 
More formally, let $U=\left[U_{1}, U_{2}\right]$ and $V=\left[V_{1}, V_{2}\right]$ be a conformal partitioning with respect to the cluster of $k$ eigenvalues in the $(1,1)$-block of $(S, T)$ :

$$
\left[\begin{array}{c}
U_{1}^{T} \\
U_{2}^{T}
\end{array}\right](A-\lambda B)\left[\begin{array}{ll}
V_{1} & V_{2}
\end{array}\right]=S-\lambda T \equiv\left[\begin{array}{cc}
S_{11} & S_{12} \\
0 & S_{22}
\end{array}\right]-\lambda\left[\begin{array}{cc}
T_{11} & T_{12} \\
0 & T_{22}
\end{array}\right] .
$$

Now, $\mathcal{L}=\operatorname{span}\left(U_{1}\right)$ and $\mathcal{R}=\operatorname{span}\left(V_{1}\right)$ form a pair of deflating subspaces associated with the cluster of $\left(S_{11}, T_{11}\right)$. Moreover, $\operatorname{span}\left(V_{1}\right)$ is a right eigenspace of $(A, B)$, and $\operatorname{span}\left(U_{2}\right)$ is a left eigenspace of $(A, B)$ associated with $\left(S_{22}, T_{22}\right)$ [26]. Indeed, we can retrieve a left eigenspace associated with $\left(S_{11}, T_{11}\right)$ and a right eigenspace associated with $\left(S_{22}, T_{22}\right)$ by a second reordering of the eigenvalues of $(S, T)$ such that, now, the "new" $\left(S_{22}, T_{22}\right)$ will correspond to the specified cluster. Moreover, by combining the $U_{i}$ 's and $V_{i}$ 's from the two reorderings it is possible to construct a block-diagonalizing equivalence transformation [20]. Let

$$
X^{-1}=\left[\begin{array}{c}
U_{2}^{(2)^{T}} \\
U_{2}^{(1)^{T}}
\end{array}\right], \quad Y=\left[\begin{array}{ll}
V_{1}^{(1)} & V_{1}^{(2)}
\end{array}\right],
$$

where $U_{i}^{(j)}$ and $V_{i}^{(j)}$ are blocks $i(=1,2)$ of $U$ and $V$ from the reordering $j(=1,2)$. Then $X^{-1}(A-\lambda B) Y$ is block-diagonal with the specified cluster in the $(1,1)$-block [20]. By construction the two block columns of $Y$ and the two block rows of $X^{-1}$ have orthonormal bases which ensure transformation matrices with optimal condition numbers [4]. Alternatively, we can block-diagonalize $(S, T)$ in (1.4) in terms of a non-orthogonal equivalence transformation directly by solving a generalized Sylvester equation (see Section 4.2.1).

In this paper we present underlying theory, algorithms and LAPACK-style software for computing a pair of deflating subspaces with specified eigenvalues of a regular matrix pair $(A, B)$ and error bounds for computed quantities (eigenvalues and eigenspaces). The error bounds are based on estimates of condition numbers for eigenvalues and eigenspaces. Typically, the algorithm for computing a pair of deflating subspaces is a two-step process. First, compute a generalized Schur form of a matrix pair $(A, B)$ using the $Q Z$ algorithm [23]. Second, reorder the specified eigenvalues to appear in the $(1,1)$-block of the generalized Schur form. The focus here is to perform the reordering (also in the real case) with a direct method [18]. A reordering method based on the periodic Schur decomposition has been proposed recently [5]. The rest of the paper is outlined as follows. In Section 1.1 we collect our notation. Section 2 gives an overview of the direct method for reordering eigenvalues in the generalized real Schur form. In Section 3 we discuss direct reordering algorithms with guaranteed backward stability. Section 4 collects theory and algorithms for computing condition numbers and error bounds for eigenvalues and deflating subspaces of a regular $(A, B)$. In Section 5 we present our Fortran 77 software for computing deflating subspaces with specified eigenvalues, condition numbers and error bounds. Some computational experiments that illustrate the accuracy and reliability of our software are presented in section 6. Finally, some conclusions are summarized in Section 7.

\section{$1.1 \quad$ Notation}

The following notation is used in the paper. $I_{n}$ denotes an identity matrix of size $n \times n$. $\lambda(A, B)$ denotes the spectrum of a regular matrix pair $(A, B)$ or pencil $A-\lambda B$. $\|A\|_{2}$ 
denotes the spectral norm (2-norm) of a matrix $A$ induced by the Euclidean vector norm. $\|A\|_{F}$ denotes the Frobenius (or Euclidean) matrix norm. $\|A\|_{M}=\max _{i, j}\left|a_{i j}\right|$, i.e. the maximum of the absolute values of the matrix entries. $\sigma_{\max }(A)$ and $\sigma_{\min }(A)$ denote the largest and smallest singular values of $A$, respectively. For a square matrix $A$ we have that $\|A\|_{2}=\sigma_{\max }(A)$ and $\left\|A^{-1}\right\|_{2}=\sigma_{\min }(A)^{-1} . A \otimes B$ denotes the Kronecker product of two matrices $A$ and $B$ whose $(i, j)$-th block element is $a_{i j} B$. The column vector $\operatorname{col}(A)$ denotes an ordered stack of the columns of $A$ from left to right starting with the first column. $A^{T}$ denotes the transpose of $A$. $A^{H}$ denotes the conjugate transpose of $A .|A|$ and $|x|$ denote the matrix and the vector whose elements are $\left|a_{i j}\right|$ and $\left|x_{i}\right|$, respectively. Inequalities such as $|A| \leq|B|,|x| \leq|y|$ are interpreted componentwise. $D=\operatorname{diag}(x)$ denotes a diagonal matrix with $d_{i i}=x_{i}$.

We frequently measure distances between subspaces as their angular distances. $\theta(x, y)$ is the (acute) angle between two 1-dimensional subspaces spanned by the vectors $x$ and $y$ :

$$
\cos \theta(x, y)=\frac{\left|x^{T} y\right|}{\|x\|_{2}\|y\|_{2}} .
$$

Generalized to the (maximum) angle between two subspaces $\mathcal{X}$ and $\mathcal{Y}$ of equal dimension $k \geq 2$ we have:

$$
\theta_{\max }(\mathcal{X}, \mathcal{Y})=\max _{x \in \mathcal{X}} \min _{y \in \mathcal{Y}} \theta(x, y)
$$

For computational purposes we use the following definition [13]:

$$
\theta_{\max }(\mathcal{X}, \mathcal{Y})=\arccos \sigma_{\min }\left(X^{T} Y\right)
$$

where the columns of $X$ and $Y$ (of size $n \times k$ ) span orthonormal bases for $\mathcal{X}$ and $\mathcal{Y}$, respectively.

\section{Direct Method for Reordering Eigenvalues in the Gener- alized Real Schur Form}

A direct reordering method for the $A-\lambda B$ problem is presented in [18], which extends and generalizes the direct SEP method in [2] to regular matrix pairs with real entries. Below we give an overview of the direct method and its numerical properties.

Without loss of generality we consider the problem of reordering the diagonal blocks of a matrix pair $(A, B)$ in the block form,

$$
A=\left[\begin{array}{cc}
A_{11} & A_{12} \\
0 & A_{22}
\end{array}\right], \quad B=\left[\begin{array}{cc}
B_{11} & B_{12} \\
0 & B_{22}
\end{array}\right],
$$

where $\left(A_{11}, B_{11}\right)$ and $\left(A_{22}, B_{22}\right)$ are of size $n_{1} \times n_{1}$ and $n_{2} \times n_{2}$, respectively, and $n_{1}, n_{2}=1$ or 2. Throughout the paper we assume that $\left(A_{11}, B_{11}\right)$ and $\left(A_{22}, B_{22}\right)$ have no eigenvalues in common, otherwise, the diagonal blocks need not be swapped.

We want to find orthonormal $Q$ and $Z$ of size $\left(n_{1}+n_{2}\right) \times\left(n_{1}+n_{2}\right)$ such that

$$
Q^{T}\left(\left[\begin{array}{cc}
A_{11} & A_{12} \\
0 & A_{22}
\end{array}\right],\left[\begin{array}{cc}
B_{11} & B_{12} \\
0 & B_{22}
\end{array}\right]\right) Z=\left(\left[\begin{array}{cc}
\hat{A}_{22} & \hat{A}_{12} \\
0 & \hat{A}_{11}
\end{array}\right],\left[\begin{array}{cc}
\hat{B}_{22} & \hat{B}_{12} \\
0 & \hat{B}_{11}
\end{array}\right]\right) \equiv(\hat{A}, \hat{B}),
$$


where $\left(A_{i i}, B_{i i}\right)$ and $\left(\hat{A}_{i i}, \hat{B}_{i i}\right)$ for $i=1,2$ are equivalent matrix pairs with the same eigenvalues but their positions are exchanged (swapped) along the block diagonal of $(A, B)$.

The direct method for constructing $Q$ and $Z$ and swapping two diagonal blocks in the generalized real Schur form of $(A, B)$ is outlined below [18]:

- Solve for $L$ and $R$ of size $n_{1} \times n_{2}$ in the generalized Sylvester equation:

$$
\begin{aligned}
& A_{11} R-L A_{22}=-A_{12}, \\
& B_{11} R-L B_{22}=-B_{12} .
\end{aligned}
$$

- Compute an orthogonal matrix $Q$ :

$$
Q^{T}\left[\begin{array}{c}
L \\
I_{n_{2}}
\end{array}\right]=\left[\begin{array}{c}
T_{L} \\
0
\end{array}\right]
$$

- Compute an orthogonal matrix $Z$ :

$$
\left[\begin{array}{ll}
I_{n_{1}} & -R
\end{array}\right] Z=\left[\begin{array}{ll}
0 & T_{R}
\end{array}\right]
$$

- Apply $Q$ and $Z$ to $(A, B)$ in an orthogonal equivalence transformation (2.2):

$$
\begin{gathered}
\left(\left[\begin{array}{cc}
\hat{A}_{22} & \hat{A}_{12} \\
0 & \hat{A}_{11}
\end{array}\right],\left[\begin{array}{cc}
\hat{B}_{22} & \hat{B}_{12} \\
0 & \hat{B}_{11}
\end{array}\right]\right) \equiv \\
\left(\left[\begin{array}{cc}
T_{L} A_{22} Z_{21} & T_{L} A_{22} Z_{22}+Q_{11}^{T} A_{11} T_{R} \\
0 & Q_{12}^{T} A_{11} T_{R}
\end{array}\right],\left[\begin{array}{cc}
T_{L} B_{22} Z_{21} & T_{L} B_{22} Z_{22}+Q_{11}^{T} B_{11} T_{R} \\
0 & Q_{12}^{T} B_{11} T_{R}
\end{array}\right]\right),
\end{gathered}
$$

where $Q$ and $Z$ are partitioned conformally with

$$
X=\left[\begin{array}{cc}
L & I_{n_{1}} \\
I_{n_{2}} & 0
\end{array}\right] \text {, and } Y=\left[\begin{array}{cc}
0 & I_{n_{2}} \\
I_{n_{1}} & -R
\end{array}\right], \quad \text { respectively }
$$

Notice that $X$ and $Y$ above are non-orthogonal transformation matrices that perform the required swapping:

$$
\left(\left[\begin{array}{cc}
A_{11} & A_{12} \\
0 & A_{22}
\end{array}\right],\left[\begin{array}{cc}
B_{11} & B_{12} \\
0 & B_{22}
\end{array}\right]\right)=X\left(\left[\begin{array}{cc}
A_{22} & 0 \\
0 & A_{11}
\end{array}\right],\left[\begin{array}{cc}
B_{22} & 0 \\
0 & B_{11}
\end{array}\right]\right) Y .
$$

To solve (2.3) we can use the generalized Schur method [21,19]. In our case, $\left(A_{i i}, B_{i i}\right), i=$ 1,2 are already in generalized Schur form and we end up solving a $2 n_{1} n_{2} \times 2 n_{1} n_{2}$ linear system $Z x=b$, where

$$
Z=\left[\begin{array}{ll}
I_{n_{2}} \otimes A_{11} & -A_{22}^{T} \otimes I_{n_{1}} \\
I_{n_{2}} \otimes B_{11} & -B_{22}^{T} \otimes I_{n_{1}}
\end{array}\right], \quad x=\left[\begin{array}{c}
\operatorname{col}(R) \\
\operatorname{col}(L)
\end{array}\right], \quad b=\left[\begin{array}{l}
-\operatorname{col}\left(A_{12}\right) \\
-\operatorname{col}\left(B_{12}\right)
\end{array}\right] .
$$

Since $n_{1}, n_{2}=1$ or 2 the linear system (2.8) will be of size $2 \times 2,4 \times 4$ or $8 \times 8$ (only $2 \times 2$ systems in the complex case). $Q$ in (2.4) and $Z$ in (2.5) can be found by using Householder 
or Givens transformations to compute a $Q R$ factorization and an $R Q$ factorization, respectively. Finally, the equivalence transformation (2.6) is just matrix-matrix multiplication and add operations on $A$ and $B$.

In the presence of rounding errors the conditioning and the solution of the generalized Sylvester equation will have the greatest impact on the stability of the direct swapping method [17]. Let $(\bar{L}, \bar{R})$ denote the computed solution of the generalized Sylvester equation (2.3), where $\bar{L}=L+\Delta L, \bar{R}=R+\Delta R$ and $(L, R)$ is the exact solution. The residuals of the computed solution are

$$
\begin{aligned}
& R_{1} \equiv A_{11} \bar{R}-\bar{L} A_{22}+A_{12}, \\
& R_{2} \equiv B_{11} \bar{R}-\bar{L} B_{22}+B_{12}
\end{aligned}
$$

Moreover, let $\bar{Q}$ and $\bar{Z}$ be the computed transformation matrices in (2.4) and (2.5). The following theorem shows how the errors in these quantities propagate to the results of the direct reordering method for swapping two $2 \times 2$ diagonal matrix pairs.

Theorem 2.1 [18] By applying the computed transformation matrices $\bar{Q}$ and $\bar{Z}$ in an equivalence transformation of $(A, B)$ we get

$$
\bar{Q}^{T} A \bar{Z}=\left[\begin{array}{cc}
\hat{A}_{22} & \hat{A}_{12} \\
0 & \hat{A}_{11}
\end{array}\right]+\left[\begin{array}{cc}
\Delta A_{22} & \Delta A_{12} \\
\Delta A_{21} & \Delta A_{11}
\end{array}\right] \equiv \hat{A}+\Delta A
$$

and

$$
\bar{Q}^{T} B \bar{Z}=\left[\begin{array}{cc}
\hat{B}_{22} & \hat{B}_{12} \\
0 & \hat{B}_{11}
\end{array}\right]+\left[\begin{array}{cc}
\Delta B_{22} & \Delta B_{12} \\
\Delta B_{21} & \Delta B_{11}
\end{array}\right] \equiv \hat{B}+\Delta B,
$$

where $\left(A_{i i}, B_{i i}\right)$ and $\left(\hat{A}_{i i}, \hat{B}_{i i}\right)$ for $i=1,2$ are equivalent matrix pairs as in (2.2) and up to first order perturbations $O\left(\|(\Delta A, \Delta B)\|_{2}\right)$ :

$$
\begin{aligned}
& \left\|\Delta A_{11}\right\|_{2} \leq \frac{1}{\left(1+\sigma_{\min ^{2}}{ }^{2}(L)\right)^{1 / 2}} \cdot \frac{\sigma_{\max }(R)}{\left(1+\sigma_{\max }^{2}(R)\right)^{1 / 2}} \cdot\left\|R_{1}\right\|_{F}, \\
& \left\|\Delta A_{21}\right\|_{2} \leq \frac{1}{\left(1+\sigma_{\min ^{2}}(L)\right)^{1 / 2}} \cdot \frac{1}{\left(1+\sigma_{\min ^{2}}{ }^{2}(R)\right)^{1 / 2}} \cdot\left\|R_{1}\right\|_{F}, \\
& \left\|\Delta A_{22}\right\|_{2} \leq \frac{\sigma_{\max }(L)}{\left(1+\sigma_{\max }{ }^{2}(L)\right)^{1 / 2}} \cdot \frac{1}{\left(1+\sigma_{\min ^{2}}(R)\right)^{1 / 2}} \cdot\left\|R_{1}\right\|_{F} .
\end{aligned}
$$

Similar bounds hold for $\left\|\Delta B_{11}\right\|_{2},\left\|\Delta B_{21}\right\|_{2},\left\|\Delta B_{22}\right\|_{2}$ with $R_{1}$ replaced by $R_{2}$.

What can we say about the size of the errors $(\Delta A, \Delta B)$ in Theorem 2.1? First of all, $\left\|\Delta A_{i j}\right\|_{2}$ and $\left\|\Delta B_{i j}\right\|_{2}$ depend on $\left\|R_{1}\right\|_{F},\left\|R_{2}\right\|_{F}$, the norms of the residuals of the computed solution $(\bar{L}, \bar{R})$ to the generalized Sylvester equation, and on the conditioning of the exact solution $(L, R)$. If $\sigma_{\min }(L)$ and $\sigma_{\min }(R)$ are small, the error can be as large as the norms of the residuals.

In [17] a perturbation analysis of the generalized Sylvester equation is presented that takes full account to the structure of the matrix equation, derives expressions for the backward error of an approximate solution $(\bar{L}, \bar{R})$, and derives condition numbers that measure the sensitivity of a solution to perturbations in $A_{11}, A_{12}, A_{22}$ and $B_{11}, B_{12}, B_{22}$, respectively. Due to the special structure of the (generalized) Sylvester equation the relation for linear 
systems "relative backward error = relative residual" [24] does not hold in general [16], [17]. Small relative backward errors will always result in small relative residuals. However, the analysis shows that for very ill-conditioned cases the norm of the relative backward errors can greatly exceed the norm of the relative residuals (in fact, by an arbitrary factor [16]). This situation appears when $(\bar{L}, \bar{R})$ is an ill-conditioned (i.e., $\sigma_{\min }(\bar{L})$ and $\sigma_{\min }(\bar{R})$ small) and large-normed (i.e., $\|(\bar{L}, \bar{R})\|_{F}$ large) solution to the generalized Sylvester equation. However, as we will see later, these situations correspond to extremely ill-conditioned eigen problems.

\section{Direct Reordering Algorithms with Guaranteed Back- ward Stability}

The error analysis of the direct method (summarized in Theorem 2.1) and numerical experiments suggest that a practical implementation should reject a swap if it would result in too large backward errors (i.e. instability). The test for stability can be performed directly and to a small extra cost. A direct algorithm with controlled backward error for swapping two diagonal blocks of a regular matrix pair $(A, B)$ in generalized real Schur form is outlined below [18]. In this section all quantities denote "computed" quantities.

\section{Direct Swapping Algorithm}

Step 1 Copy $A$ and $B$ to $S$ and $T$, respectively.

$$
S \leftarrow A, T \leftarrow B
$$

Step 2 Solve for $(L, R)$ in the generalized Sylvester equation:

$$
\begin{aligned}
& S_{11} R-L S_{22}=-S_{12} \gamma, \\
& T_{11} R-L T_{22}=-T_{12} \gamma .
\end{aligned}
$$

Use Gaussian elimination with complete pivoting to solve the corresponding linear system and a scaling factor $\gamma$ to prevent against overflow [19].

Step 3 Compute an orthogonal matrix $Q$ :

$$
Q^{T}\left[\begin{array}{c}
L \\
\gamma I_{n_{2}}
\end{array}\right]=\left[\begin{array}{c}
T_{L} \\
0
\end{array}\right] .
$$

Use Householder transformations to compute a $Q R$ factorization [13], [1].

Step 4 Compute an orthogonal matrix $Z$ :

$$
\left[\begin{array}{ll}
\gamma I_{n_{1}} & -R
\end{array}\right] Z=\left[\begin{array}{ll}
0 & T_{R}
\end{array}\right]
$$

Use Householder transformations to compute an $R Q$ factorization [13], [1]. 
Step 5 Perform the swapping tentatively with backward stability test:

$$
S \leftarrow Q^{T} S Z \equiv\left[\begin{array}{ll}
S_{11} & S_{12} \\
S_{21} & S_{22}
\end{array}\right], \quad T \leftarrow Q^{T} T Z \equiv\left[\begin{array}{ll}
T_{11} & T_{12} \\
T_{21} & T_{22}
\end{array}\right] .
$$

Step 6 If the swap is accepted, apply the equivalence transformation to $(A, B)$ :

$$
A \leftarrow S, \quad B \leftarrow T \text {. }
$$

Set the $(2,1)$-blocks to zero.

Step 7 Standardize existing $2 \times 2$ blocks.

Use the LAPACK routine _HGEQZ to standardize and (possibly) separate $2 \times 2$ blocks further [1].

The backward stability test in step 5 is split in two parts:

Weak stability test: Check if $\left\|\left(S_{21}, T_{21}\right)\right\|_{F} \leq$ tol1.

Strong stability test: Check if $\left\|\left(A-Q S Z^{T}, B-Q T Z^{T}\right)\right\|_{F} \leq$ told.

The size of the $(2,1)$-blocks are most crucial since their norms immediately reflect the stability of the swapping. Indeed, $\left\|\left(S_{21}, T_{21}\right)\right\|_{F}$ is the size of the optimal backward perturbation $(E, F)$ of the reordered $(S, T)$ such that $(S+E, T+F)$ has the $n_{2}$ first columns of $Q$ and $Z$ as an exact pair of deflating subspaces (see Section 4.2.4 and [28]). The size of the $(2,1)$-blocks are controlled by toll in the weak stability test and they should not exceed $O\left(\epsilon\|(S, T)\|_{F}\right)$, where $\epsilon$ is the relative machine precision. The strong stability test takes all backward errors into account and by choosing tol 2 of the size $O\left(\epsilon\|(A, B)\|_{F}\right)$ and rejecting the swap if the error is larger than tol, we obtain guaranteed backward stability. If both the weak and the strong stability tests hold then the swap is accepted, otherwise rejected. We have used $10 \epsilon\|(A, B)\|_{F}$ for both toll and told.

One could argue that it is enough with the weak stability test and in fact we have (so far) not been able to construct any example where the strong test fails while the weak test does not. However, since the extra cost of the strong stability test is only marginal it is included in our software.

After step 2 it would be possible to compute an optimal block-diagonalizing equivalence transformation that minimizes the condition numbers of the transformation matrices [6],[20]. Since the scaling factors (which possibly are large numbers) will show up in the $S_{i j}$ and $T_{i j}$ blocks, we do not expect any substantial improvements in performing this block-diagonal scaling. Computational experiments in Matlab confirm this statement too.

The swapping of a $2 \times 2$ block and a $1 \times 1$ block (or vice versa) is performed similarly as swapping two $2 \times 2$ blocks. However, the swapping of two $1 \times 1$ blocks is performed using orthogonal (unitary) Givens rotations [30]. In the complex case we perform all reordering with unitary Givens rotations. 


\subsection{Justification for Rejecting a Swap}

It is well-known that the generalized eigenvalue problem (as well as the standard unsymmetric problem) is potentially ill-conditioned in the sense that eigenvalues and eigenspaces may change drastically even under small perturbations of the data (e.g., see [26], [9]). If we insist on performing a reordering of $\left(S_{11}, T_{11}\right)$ and $\left(S_{22}, T_{22}\right)$ for an ill-conditioned problem, we may destroy any spectral information in $A-\lambda B$. Our computational experiments show that close eigenvalues or small separation between $\left(S_{11}, T_{11}\right)$ and $\left(S_{22}, T_{22}\right)$ are not enough for rejecting a swap. It is the sensitivity of the eigenspaces that matters most, which in turn is perfectly signaled by the norm of the solutions $L$ and $R$ to the associated generalized Sylvester equation. As before, we illustrate with the case $n_{1}=n_{2}=2$. From (2.7) we get that after the reordering

$$
\mathcal{L}_{1}=\operatorname{span}\left(\left[\begin{array}{c}
I_{2} \\
0
\end{array}\right]\right), \quad \mathcal{R}_{1}=\operatorname{span}\left(\left[\begin{array}{c}
I_{2} \\
0
\end{array}\right]\right),
$$

and

$$
\mathcal{L}_{2}=\operatorname{span}\left(\left[\begin{array}{c}
L \\
I_{2}
\end{array}\right]\right), \quad \mathcal{R}_{2}=\operatorname{span}\left(\left[\begin{array}{c}
R \\
I_{2}
\end{array}\right]\right),
$$

are pairs of deflating subspaces associated with the spectrum of the reordered blocks $\left(S_{11}, T_{11}\right)$ and $\left(S_{22}, T_{22}\right)$, respectively. Informally, we see that when $\|L\|$ and $\|R\|$ are large enough $\mathcal{L}_{i}$ for $i=1,2$ and $\mathcal{R}_{i}$ for $i=1,2$ are almost linearly dependent. More formally; by partitioning $Q$ in (2.4) appropriately and using properties of the $C S$ decomposition of $Q$, we can show that

$$
\cos \theta_{\max }\left(\mathcal{L}_{1}, \mathcal{L}_{2}\right)=\left\|Q_{11}\right\|_{2}=\frac{\sigma_{\max }(L)}{\left(1+\sigma_{\max }^{2}(L)\right)^{1 / 2}}
$$

Now, $\theta_{\max }\left(\mathcal{L}_{1}, \mathcal{L}_{2}\right)$ is close to zero if and only if $\|L\|_{2}$ is large. A small largest angle between $\mathcal{L}_{1}$ and $\mathcal{L}_{2}$, means that the left deflating subspaces associated with $\left(S_{11}, T_{11}\right)$ and $\left(S_{22}, T_{22}\right)$ are almost linearly dependent. Similarly, it can be shown that $\|R\|_{2}$ is large if and only if $\theta_{\max }\left(\mathcal{R}_{1}, \mathcal{R}_{2}\right)$ is close to zero.

\subsection{Algorithm Variants for the Stability Tests}

In the following we present some different variants to perform the stability tests for accepting or rejecting a swap of two $2 \times 2$ diagonal matrix pairs.

Method 1: Perform the weak and strong stability tests on $(S, T)$ as computed in step 5. Here $S_{21}$ and $T_{21}$ are full $2 \times 2$ blocks with possibly non-zero entries. If the swap is accepted then set $S_{21}$ and $T_{21}$ to zero.

Method 2: Triangularize $T$ (from step 5) with an orthogonal $U$ from the left and apply the transformation to $S$, i.e.

$$
S \leftarrow U^{T} S \equiv\left[\begin{array}{ll}
S_{11} & S_{12} \\
S_{21} & S_{22}
\end{array}\right], \quad T \leftarrow U^{T} T \equiv\left[\begin{array}{cc}
T_{11} & T_{12} \\
0 & T_{22}
\end{array}\right] .
$$

Now $T$ is upper triangular and $T_{21}$ is a zero $2 \times 2$ block, while $S_{21}$ is still a full matrix block. We also have the freedom to triangularize $T$ (from step 5 ) with an orthogonal $V$ from the 
right and apply the transformation to $S$ similarly. The triangularization method (from left or right) that produces the $(2,1)$-block of $S$ with smallest (Frobenius) norm is chosen and checked for stability. If the swap is accepted, then $S_{21}$ is set to zero.

Method 3: Transform $(S, T)$ (from step 5) to generalized Hessenberg form:

$$
(S, T) \leftarrow Q_{H}^{T}(S, T) Z_{H} \equiv\left(\left[\begin{array}{ll}
S_{11} & S_{12} \\
S_{21} & S_{22}
\end{array}\right],\left[\begin{array}{cc}
T_{11} & T_{12} \\
0 & T_{22}
\end{array}\right]\right),
$$

where $S_{21}$ only has one non-zero element, namely in its $(1,2)$ position. Notice that we cannot guarantee that this element is small, even if the original $S_{21}$ is small. We apply the $Q Z$ algorithm to $(S, T)$ in generalized Hessenberg form giving

$$
(S, T)-Q_{Q Z}^{T}(S, T) Z_{Q Z} \equiv\left(\left[\begin{array}{cc}
S_{11} & S_{12} \\
0 & S_{22}
\end{array}\right],\left[\begin{array}{cc}
T_{11} & T_{12} \\
0 & T_{22}
\end{array}\right]\right) .
$$

If the strong stability test holds, then the swap is accepted, otherwise rejected.

Methods 1-2 are direct methods and they differ in the way we transform the matrix pair $(S, T)$ before we apply the stability tests. Our aim is to discard as little information as possible in the reordered matrix pair when we impose the $(2,1)$-blocks to be zero. Method 1 is the generic variant and we discard information in both $S$ and $T$. In method 2 we only discard information in the $S$-part. Let $\tilde{S}_{i j}$ and $\tilde{T}_{i j}$ denote the $(i, j)$-blocks of $S$ and $T$ before the triangularization of $T$ and $U_{i j}\left(V_{i j}\right)$ the corresponding blocks of the orthogonal (unitary) transformation $U$ (or $V$ ) used to triangularize from left (or right). The triangularization of $T$ from left results in $T_{21}=0$ and $S_{21}=U_{22}^{T}\left(\tilde{S}_{21}-\tilde{T}_{21} \tilde{T}_{11}^{-1} \tilde{S}_{11}\right)$, with

$$
\left\|S_{21}\right\|_{2} \leq\left\|\tilde{S}_{21}-\tilde{T}_{21} \tilde{T}_{11}^{-1} \tilde{S}_{11}\right\|_{F}
$$

Similarly, the triangularization from right results in $T_{21}=0$ and $S_{21}=\left(\tilde{S}_{21}-\tilde{S}_{22} \tilde{T}_{22}^{-1} \tilde{T}_{21}\right) V_{11}^{T}$, with

$$
\left\|S_{21}\right\|_{2} \leq\left\|\tilde{S}_{21}-\tilde{S}_{22} \tilde{T}_{22}^{-1} \tilde{T}_{21}\right\|_{F}
$$

Roughly speaking, if the bound (3.1) is smaller than the bound (3.2), then triangularization from the left is to prefer, otherwise from the right. We have computational evidence that the exact expressions for $\left\|S_{21}\right\|$ in (3.1) and (3.2) (and the bounds) can be smaller than the information we discard in method 1 (see bounds for $\Delta A_{21}$ and $\Delta B_{21}$ in Theorem 2.1).

The expressions for $S_{21}$ above can be traced back to expressions involving blocks of $S$ and $T$ before the reordering (i.e. the original $A_{i j}$ and $B_{i j}(2.1)$ ) and the residuals of the generalized Sylvester equation in step $2\left(R_{1}=A_{11} R-L A_{22}+\gamma A_{12}\right.$ and $R_{2}=B_{11} R-L B_{22}+$ $\gamma B_{12}$ ), resulting in the following bounds on the block $S_{21}$ of $S$ after the triangularization in method 2:

Corollary 3.1 (i) Let $S-U^{T} S$, where $U$ is the exact orthogonal transformation that triangularizes $T$ from the left. Then (up to first order perturbations)

$$
\left\|\Delta A_{21}\right\|_{2} \equiv\left\|S_{21}\right\|_{2} \leq \frac{\left\|R_{1}-R_{2} B_{22}^{-1} A_{22}\right\|_{F}}{\left(1+\sigma_{\min ^{2}}^{2}(L)\right)^{1 / 2}\left(1+\sigma_{\min }^{2}(R)\right)^{1 / 2}}
$$


(ii) Similarly, let $S-S V$, where $V$ is the exact orthogonal transformation that triangularizes $T$ from the right. Then (up to first order perturbations)

$$
\left\|\Delta A_{21}\right\|_{2} \equiv\left\|S_{21}\right\|_{2} \leq \frac{\left\|R_{1}-A_{11} B_{11}{ }^{-1} R_{2}\right\|_{F}}{\left(1+\sigma_{\min }^{2}(L)\right)^{1 / 2}\left(1+\sigma_{\min }^{2}(R)\right)^{1 / 2}} .
$$

In both cases $\Delta B_{21} \equiv T_{21}=0$.

Proof From the proof of Theorem 2.1 [18], we have (up to first order perturbations) $\tilde{S}_{21}=Q_{12}^{T} R_{1} Z_{21}$ and $\tilde{T}_{21}=Q_{12}^{T} R_{2} Z_{21}$, where $Q_{i j}$ and $Z_{i j}$ are blocks of $Q$ and $Z$ in the orthogonal equivalence transformation $(2.2)$ that performs the reordering of two diagonal blocks. Moreover, $\left\|Z_{21}\right\|_{2}=1 /\left(1+\sigma_{\min }^{2}(R)\right)^{1 / 2}$ and $\left\|Q_{12}^{T}\right\|_{2}=1 /\left(1+\sigma_{\min }^{2}(L)\right)^{1 / 2}$ [18]. From (2.6) we get $\tilde{S}_{11}=T_{L} A_{22} Z_{21}$ and $\tilde{T}_{11}=T_{L} B_{22} Z_{21}$. Using these expressions in $S_{21}=U_{22}^{T}\left(\tilde{S}_{21}-\tilde{T}_{21} \tilde{T}_{11}^{-1} \tilde{S}_{11}\right)$ we get $S_{21}=U_{22}^{T} Q_{12}^{T}\left(R_{1}-R_{2} B_{22}{ }^{-1} A_{22}\right) Z_{21}$, and (3.3) follows from well-known inequalities for matrix norms. The bound (3.4) can be proved similarly.

Besides backward stability of the reordering we strive to affect the eigenvalues as little as possible. We have constructed examples where the triangularization (from left or right) of $S$ has great impact on the reordered eigenvalues.

In method 3 we let the $Q Z$ algorithm decide what information to discard, and hopefully this should give us accurate eigenvalues after the swapping of two diagonal blocks. However, applying the $Q Z$ algorithm also means that the method may be iterative in the last step. In the worst case, already ordered eigenvalues may be reordered again. We use this variant mainly for comparing results between our direct methods and a "best possible" hybrid method.

\section{Condition Numbers and Error Bounds for Eigenvalues and Eigenspaces of a Regular $(A, B)$}

A condition number of a problem measures the sensitivity of the solution to small changes in the problem data. The problem is ill-conditioned if the condition number is large, and ill-posed if the condition number is infinite. Condition numbers can be used to bound errors in computed quantities (e.g., eigenvalues, eigenvectors and deflating subspaces). We construct error bounds from forward perturbation bounds for the problem (that define our condition numbers) and the knowledge of the backward error corresponding to the computed solution. The best we can ask for is to have an explicit expression for the optimal backward error related to the residual of the computed solution. A residual-based expression of the optimal backward error is algorithm-independent, i.e. it can be applied to an approximate solution resulting from any algorithm used to solve the problem. Otherwise, we have to rely to an upper bound on the backward error, resulting from a backward error analysis of the algorithm used to compute the solution. If we use backward stable methods to compute an eigendecomposition of a regular $(A, B)$, then we know that the norm of the backward error in $(A+E, B+F)$ is $\|(E, F)\|=O(\epsilon\|(A, B)\|)$, where $\epsilon$ is the relative machine accuracy.

Condition numbers may be very expensive to compute and therefore we will use inexpensive estimates. In the extreme case the exact condition number (separation between two 
matrix pairs) is an $O\left(n^{6}\right)$ operation while the estimate is computed in only $O\left(n^{3}\right)$ operations [19]. Condition estimators are by definition approximations or bounds to the exact values they try to estimate, and may therefore occasionally overestimate or underestimate the true condition number by a large factor. Extensive computational experiments (on moderately sized problems) show that this seldom happens, but it is of course always possible to construct counter examples.

The condition numbers and estimates computed by our software and discussed here are reciprocal values of a condition number for an individual eigenvalue (or a cluster of eigenvalues), a condition number for an eigenvector (or eigenspace), and spectral projectors onto a selected cluster. By computing reciprocal values we avoid overflow. An infinite value or a condition number that would overflow are reported by the reciprocal value zero. This is in agreement with the condition estimation for the standard eigenvalue problem in LAPACK [3].

These quantities appear in error bounds for eigenvalues and eigenspaces of a regular $(A, B)$, which we also review here. In agreement with the standard eigenvalue problem in LAPACK we measure changes in eigenvectors or eigenspaces by their change in angle. Moreover, our condition numbers yield both asymptotic and global error bounds. The asymptotic bounds are only accurate for small perturbations $(E, F)$ of $(A, B)$, while the global bounds work for all $\|(E, F)\|$ up to a certain bound. The size of this bound is determined by the conditioning of the problem and may therefore be large (for a wellconditioned problem) or small (for an ill-conditioned problem). We also show how these upper bounds can be estimated. Finally, we present some new results (due to Sun [28]) that give us explicit expressions for the optimal backward error related to the residual of a computed eigenspace, and which also lead to an residual-based global angular error bound for computed left and right deflating subspaces.

\subsection{A Condition Number and Error Bounds for Simple Eigenvalues}

Assume that $(\alpha, \beta) \neq(0,0)$ is a simple eigenvalue of a regular matrix pair $(A, B)$ with left and right eigenvectors $y$ and $x$, respectively, satisfying (1.1). Notice that all non-zero scalar multiples of $(\alpha, \beta)$ is also an eigenvalue of $(A, B)$. Therefore, it is natural to regard the subspace spanned by the vector $(\alpha, \beta)^{T}$ as the generalized eigenvalue of $(A, B)[26]$ :

$$
<\alpha, \beta>=\left\{\tau(\alpha, \beta)^{T}: \tau \in \mathbf{C},(\alpha, \beta) \neq(0,0)\right\} .
$$

In the perturbation theory for generalized eigenvalues we consider the distance between pairs $(\alpha, \beta)$ and $\left(\alpha^{\prime}, \beta^{\prime}\right)$. A useful metric is the chordal distance of two pairs defined as

$$
\mathcal{X}\left((\alpha, \beta),\left(\alpha^{\prime}, \beta^{\prime}\right)\right)=\frac{\left|\alpha \beta^{\prime}-\beta \alpha^{\prime}\right|}{\sqrt{|\alpha|^{2}+|\beta|^{2}} \sqrt{\left|\alpha^{\prime}\right|^{2}+\left|\beta^{\prime}\right|^{2}}} .
$$

If we set $\lambda=\alpha / \beta$ and $\lambda^{\prime}=\alpha^{\prime} / \beta^{\prime}$, then we have

$$
\mathcal{X}\left(\lambda, \lambda^{\prime}\right)=\frac{\left|\lambda-\lambda^{\prime}\right|}{\sqrt{1+|\lambda|^{2}} \sqrt{1+\left|\lambda^{\prime}\right|^{2}}} .
$$

Some characteristics of the chordal metric are summarized below [26]: The point at infinity is no more than unit distance from any other point $\left(\mathcal{X}(\lambda, \infty)=1 / \sqrt{1+|\lambda|^{2}} \leq 1\right)$. If $|\lambda|,\left|\lambda^{\prime}\right| \leq$ 
1, then $\mathcal{X}\left(\lambda, \lambda^{\prime}\right)$ behaves essentially like the Euclidean metric. The chordal distance between two large numbers can be small (e.g., $\mathcal{X}(\lambda, 2 \lambda) \approx 1 /|\lambda|$, when $\lambda \rightarrow \infty$ ). Accordingly, large numbers can have very small chordal distances, even when they have large relative errors.

In the following we review how to measure the sensitivity of simple eigenvalues of a regular matrix pair. Let $(\alpha, \beta)$ be a simple eigenvalue of $(A, B)$ with left and right eigenvectors $y$ and $x$, respectively. Let $(E, F)$ be a perturbation of $(A, B)$, and $\|(E, F)\|_{2}=\epsilon_{2}$. Then there is an eigenvalue $\left(\alpha^{\prime}, \beta^{\prime}\right)$ of $(A+E, B+F)$ such that the following first order bound holds [26]:

$$
\mathcal{X}\left((\alpha, \beta),\left(\alpha^{\prime}, \beta^{\prime}\right)\right) \leq \nu \cdot \epsilon_{2}+O\left(\epsilon_{2}^{2}\right)
$$

where $\nu$ is the condition number for a simple generalized eigenvalue:

$$
\nu=\frac{\|x\|_{2}\|y\|_{2}}{\sqrt{\left|y^{H} A x\right|^{2}+\left|y^{H} B x\right|^{2}}} .
$$

Notice that $y^{H} A x / y^{H} B x$ is equal to $\lambda=\alpha / \beta$. By using $y^{H} A x$ and $y^{H} B x$ in (4.4), the condition number is independent of the normalization of the eigenvectors and the corresponding eigenvalue pair. Deleting higher order terms in (4.3) we get an asymptotic error bound for a simple eigenvalue:

$$
\mathcal{X}\left((\alpha, \beta),\left(\alpha^{\prime}, \beta^{\prime}\right)\right) \lesssim \nu\|(E, F)\|_{2}
$$

By replacing $\|(E, F)\|_{2}$ by $\|(E, F)\|_{F}$ in (4.5) we get a somewhat weaker but a more computationally attractive bound.

The following example illustrates the definition of $\nu$. Let

$$
\begin{aligned}
Y^{H} & =\left[\begin{array}{cc}
1 & 2 \delta^{-1} \\
0 & 1
\end{array}\right], \quad X=\left[\begin{array}{cc}
1 & \delta^{-1} \\
0 & 1
\end{array}\right], \quad \text { and } \\
Y^{H} A X & =\left[\begin{array}{cc}
\epsilon & 0 \\
0 & -2 \epsilon
\end{array}\right], \quad Y^{H} B X=\left[\begin{array}{cc}
\epsilon & 0 \\
0 & \epsilon
\end{array}\right], \quad \text { i.e. } \\
A & =\left[\begin{array}{cc}
\epsilon & 3 \epsilon \delta^{-1} \\
0 & -2 \epsilon
\end{array}\right], \quad B=\left[\begin{array}{cc}
\epsilon & -3 \epsilon \delta^{-1} \\
0 & \epsilon
\end{array}\right] .
\end{aligned}
$$

By choosing $\epsilon>0$ and $\delta>0$ small we get $\nu_{i}=O\left(\epsilon^{-1} \delta^{-1}\right)$ for $i=1,2$ from (4.4) and $X$ and $Y^{H}$ above.

The eigenvalues of $(A, B)$ are $1(=\epsilon / \epsilon)$ and $-2(=-2 \epsilon / \epsilon)$. Now, we consider the equivalent pencil

$$
\left(D^{-1} A, D^{-1} B\right)=\left(\left[\begin{array}{cc}
1 & 3 \delta^{-1} \\
0 & -2
\end{array}\right],\left[\begin{array}{cc}
1 & 3 \delta^{-1} \\
0 & 1
\end{array}\right]\right)
$$

where

$$
D=\left[\begin{array}{cc}
\epsilon^{-1} & 0 \\
0 & \epsilon^{-1}
\end{array}\right]
$$


It has the same eigenvalues (and eigenvectors too!), but their individual condition numbers are only $\nu_{i}=O\left(\delta^{-1}\right)$ for $\epsilon \approx \delta>0$, showing that the eigenvalues 1 and -2 are better conditioned for the equivalent pencil.

There also exist global error bounds which are not limited by the size of $\|(E, F)\|$ (e.g., Bauer-Fike-style error bounds). In the following we assume that $(A, B)$ is a diagonalizable matrix pair such that

$$
Y^{H}(A, B) X=\left(\operatorname{diag}\left(\alpha_{i}\right), \operatorname{diag}\left(\beta_{i}\right)\right) .
$$

Let $Y^{H}$ and $X$ be normalized such that $\left|\alpha_{i}\right|^{2}+\left|\beta_{i}\right|^{2}=1$ and $\left\|y_{i}\right\|_{2}=1$, i.e. we overwrite $y_{i}$ with $y_{i} /\left\|y_{i}\right\|_{2},\left(\alpha_{i}, \beta_{i}\right)$ with $\left(\alpha_{i}, \beta_{i}\right) /\left(\left|\alpha_{i}\right|^{2}+\left|\beta_{i}\right|^{2}\right)^{1 / 2}$ and, finally, $x_{i}$ with $x_{i} \| y_{i}||_{2} /\left(\left|\alpha_{i}\right|^{2}+\right.$ $\left.\left|\beta_{i}\right|^{2}\right)^{1 / 2}$. With this normalization the individual condition number for $\left(\alpha_{i}, \beta_{i}\right)$ is $\nu_{i}=\left\|x_{i}\right\|_{2}$.

Let $\left(\alpha^{\prime}, \beta^{\prime}\right)$ with $\left|\alpha^{\prime}\right|^{2}+\left|\beta^{\prime}\right|^{2}=1$ be an eigenvalue of $(A+E, B+F)$. Then we have the following Bauer-Fike-style bound [8]:

$$
\min _{i} \mathcal{X}\left(\left(\alpha^{\prime}, \beta^{\prime}\right),\left(\alpha_{i}, \beta_{i}\right)\right) \equiv \min _{i}\left|\alpha_{i} \beta^{\prime}-\beta_{i} \alpha^{\prime}\right| \leq\left\|Y^{H}\right\|_{2}\|X\|_{2}\|(E, F)\|_{F} .
$$

If $X$ and $Y$ are normalized as above, then $\left\|Y^{H}\right\|_{2} \leq \sqrt{n}$ and $\|X\|_{2} \leq \sqrt{n} \max _{i} \nu_{i}$, which in (4.7) give us a Bauer-Fike-style bound for the generalized eigenvalue problem:

$$
\min _{i} \mathcal{X}\left(\left(\alpha^{\prime}, \beta^{\prime}\right),\left(\alpha_{i}, \beta_{i}\right)\right) \leq n \max _{i} \nu_{i}\|(E, F)\|_{F} \text {. }
$$

As for the standard eigenvalue problem, it is the most ill-conditioned eigenvalue that determines the size of the error bound. In words, (4.7) and (4.8) bound the smallest distance (measured in the chordal metric) between an eigenvalue of the perturbed and unperturbed matrix pairs.

It is possible to strengthen the classical Bauer-Fike bound (for the standard problem) giving a bound for each individual eigenvalue [7,3], whose size is determined by the conditioning of the individual eigenvalue. By applying the same technique (under the same assumptions as for (4.8) ) we can prove that any eigenvalue $\left(\alpha^{\prime}, \beta^{\prime}\right)$ with $\left|\alpha^{\prime}\right|^{2}+\left|\beta^{\prime}\right|^{2}=1$ of $(A+E, B+F)$ must lie in one of the regions ("balls")

$$
\left\{(\alpha, \beta),|\alpha|^{2}+|\beta|^{2}=1: \mathcal{X}\left((\alpha, \beta),\left(\alpha_{i}, \beta_{i}\right)\right) \leq n \nu_{i}\|(E, F)\|_{2}\right\}
$$

Notice that the sizes of the regions (bounds) are only a factor $n$ larger than the first order error bound (4.5). Moreover, the global error bound (4.9) with respect to an eigenvalue $\left(\alpha_{i}, \beta_{i}\right)$ is only useful if it defines a region that does not intersect with regions corresponding to other eigenvalues. If two or more regions intersect, then we can only say that an eigenvalue of the perturbed matrix pair lies in the union of the overlapping regions.

There also exist other Bauer-Fike-style bounds for the generalized eigenvalue problem and other generalizations (see [26] for a review and further references).

\subsection{Conditioning and Error Bounds for Left and Right Deflating Sub- spaces Associated with a Cluster of Eigenvalues}

If $(A, B)$ has $n$ distinct eigenvalues, then there exist non-singular matrices $Y$ and $X$ that transform $(A, B)$ to diagonal form (4.6). Moreover, their columns $y_{i}$ and $x_{i}$ are left and right eigenvectors associated with the eigenvalues $\left(\alpha_{i}, \beta_{i}\right)(i=1: n)$. Let $Y^{H} \equiv P^{-1}$ and 
from (4.6) we have that $A X+B X=P \operatorname{diag}\left(\alpha_{i}+\beta_{i}\right)$, i.e. the columns $p_{i}$ and $x_{i}$ of $P$ and $X$, respectively, span pairs of one-dimensional left and right deflating subspaces. Accordingly, conditioning and error bounds for individual eigenvectors can be regarded as a special case of error bounds for left and right deflating subspaces.

Without loss of generality we assume that $(A, B)$ is in generalized Schur form

$$
A=\left[\begin{array}{cc}
A_{11} & A_{12} \\
0 & A_{22}
\end{array}\right], \quad B=\left[\begin{array}{cc}
B_{11} & B_{12} \\
0 & B_{22}
\end{array}\right] \text {. }
$$

In the following we review condition numbers and error bounds for left and right deflating subspaces associated with the cluster of $m(1 \leq m \leq n-1)$ eigenvalues (counting multiplicities) of $\left(A_{11}, B_{11}\right)$. To explain the bounds we need to introduce some definitions.

\subsubsection{Block-diagonalization and Separation of Two Matrix Pairs}

An equivalence transformation that block-diagonalizes $(A, B)$ in generalized Schur form (4.10) can be expressed as

$$
\begin{aligned}
{\left[\begin{array}{cc}
I_{m} & -L \\
0 & I_{n-m}
\end{array}\right] } & \left(\left[\begin{array}{cc}
A_{11} & A_{12} \\
0 & A_{22}
\end{array}\right],\left[\begin{array}{cc}
B_{11} & B_{12} \\
0 & B_{22}
\end{array}\right]\right)\left[\begin{array}{cc}
I_{m} & R \\
0 & I_{n-m}
\end{array}\right]= \\
& \left(\left[\begin{array}{cc}
A_{11} & 0 \\
0 & A_{22}
\end{array}\right],\left[\begin{array}{cc}
B_{11} & 0 \\
0 & B_{22}
\end{array}\right]\right) .
\end{aligned}
$$

Solving for $(L, R)$ in $(4.11)$ is equivalent to solve the generalized Sylvester equation

$$
\begin{aligned}
& A_{11} R-L A_{22}=-A_{12}, \\
& B_{11} R-L B_{22}=-B_{12},
\end{aligned}
$$

which can be rewritten as a $2 m(n-m) \times 2 m(n-m)$ linear system $Z_{u} x=b$, where

$$
Z_{u}=\left[\begin{array}{ll}
I_{n-m} \otimes A_{11} & -A_{22}^{T} \otimes I_{m} \\
I_{n-m} \otimes B_{11} & -B_{22}^{T} \otimes I_{m}
\end{array}\right]
$$

and

$$
x=\left[\begin{array}{c}
\operatorname{col}(R) \\
\operatorname{col}(L)
\end{array}\right], \quad b=\left[\begin{array}{l}
-\operatorname{col}\left(A_{12}\right) \\
-\operatorname{col}\left(B_{12}\right)
\end{array}\right] .
$$

Moreover, let

$$
p=\left(1+\|L\|_{F}^{2}\right)^{1 / 2}, \quad q=\left(1+\|R\|_{F}^{2}\right)^{1 / 2} .
$$

In the perturbation theory for the generalized eigenvalue problem, $p$ and $q$ play the same role as the norm of the spectral projector does for the standard eigenvalue problem [9]. Indeed, if $B=I$, then $p=q$ and $p$ equals the norm of the projection onto an invariant subspace of $A$. For the generalized eigenvalue problem we need both a left and a right projection norm since the left and right deflating subspaces are (normally) different. 
Another important quantity involved in the sensitivity analysis of deflating subspaces (and eigenvalues) is the separation of two matrix pairs $\left(A_{11}, B_{11}\right)$ and $\left(A_{22}, B_{22}\right)$ [25]:

$$
\operatorname{Dif}_{u}\left[\left(A_{11}, B_{11}\right),\left(A_{22}, B_{22}\right)\right]=\inf _{\|(L, R)\|_{F}=1}\left\|\left(A_{11} R-L A_{22}, B_{11} R-L B_{22}\right)\right\|_{F} .
$$

The generalized Sylvester operator $\left(A_{11} R-L A_{22}, B_{11} R-L B_{22}\right)$ in the definition of Dif ${ }_{u}$ is obtained from block-diagonalizing a regular matrix pair in upper block triangular form. Dif $u$ is a generalization of the separation between two matrices $\left(\operatorname{Sep}\left(A_{11}, A_{22}\right)=\sigma_{\min }\left(I_{n-m} \otimes\right.\right.$ $\left.\left.A_{11}-A_{22}^{T} \otimes I_{m}\right)[25]\right)$ to two matrix pairs and it measures the separation of their spectra in the following sense. If $\left(A_{11}, B_{11}\right)$ and $\left(A_{22}, B_{22}\right)$ have a common eigenvalue, then Dif $u$ is zero and it is small if there is a small perturbation of either $\left(A_{11}, B_{11}\right)$ or $\left(A_{22}, B_{22}\right)$ that makes them have a common eigenvalue.

From the matrix representation (4.13) of the generalized Sylvester operator it can be shown [9] that

$$
\operatorname{Dif}_{u}\left[\left(A_{11}, B_{11}\right),\left(A_{22}, B_{22}\right)\right]=\sigma_{\min }\left(Z_{u}\right) .
$$

Moreover, it follows that the generalized Sylvester equation has a unique solution if and only if $\operatorname{Dif}_{u}>0$ and we can bound the norm of $(L, R)$ as

$$
\|(L, R)\|_{F} \leq \frac{\left\|\left(A_{12}, B_{12}\right)\right\|_{F}}{\operatorname{Dif}_{u}}
$$

Notice that $\operatorname{Dif}_{u}\left[\left(A_{22}, B_{22}\right),\left(A_{11}, B_{11}\right)\right]$ does not generally equal $\operatorname{Dif}_{u}\left[\left(A_{11}, B_{11}\right),\left(A_{22}, B_{22}\right)\right]$ (unless $A_{i i}$ and $B_{i i}$ are symmetric for $i=1,2$ ). Accordingly, the ordering of the arguments plays a role for the separation of two matrix pairs, while it does not for the separation of two matrices $\left(\operatorname{Sep}\left(A_{11}, A_{22}\right)=\operatorname{Sep}\left(A_{22}, A_{11}\right)\right)$. Therefore, we introduce the notation

$$
\operatorname{Dif}_{l}\left[\left(A_{11}, B_{11}\right),\left(A_{22}, B_{22}\right)\right]=\operatorname{Dif}_{u}\left[\left(A_{22}, B_{22}\right),\left(A_{11}, B_{11}\right)\right] \text {. }
$$

An associated generalized Sylvester operator $\left(A_{22} R-L A_{11}, B_{22} R-L B_{11}\right)$ in the definition of Dif $l$ is obtained from block-diagonalizing a regular matrix pair in lower block triangular form:

$$
\begin{aligned}
{\left[\begin{array}{cc}
I_{m} & 0 \\
-L & I_{n-m}
\end{array}\right] } & \left(\left[\begin{array}{cc}
A_{11} & 0 \\
A_{21} & A_{22}
\end{array}\right],\left[\begin{array}{cc}
B_{11} & 0 \\
B_{21} & B_{22}
\end{array}\right]\right)\left[\begin{array}{cc}
I_{m} & 0 \\
R & I_{n-m}
\end{array}\right]= \\
& \left(\left[\begin{array}{cc}
A_{11} & 0 \\
0 & A_{22}
\end{array}\right],\left[\begin{array}{cc}
B_{11} & 0 \\
0 & B_{22}
\end{array}\right]\right) .
\end{aligned}
$$

\subsubsection{Conditioning of Left and Right Deflating Subspaces}

Assume that $(A, B)$ is in generalized Schur form (4.10) and that $\left(A_{11}, B_{11}\right)$ contains the cluster of $m$ eigenvalues with left and right deflating subspaces $\mathcal{L}$ and $\mathcal{R}$, respectively. Typically, $\mathcal{L}=\operatorname{span}\left\{U_{1}\right\}$ and $\mathcal{R}=\operatorname{span}\left\{V_{1}\right\}$ where $U_{1}$ and $V_{1}$ are the leading $m$ columns of the unitary (orthogonal) $U$ and $V$ in (1.2) that transform $(A, B)$ to generalized Schur form. Furthermore, let $\mathcal{L}^{\prime}=\operatorname{span}\left\{U^{\prime}{ }_{1}\right\}$ and $\mathcal{R}^{\prime}=\operatorname{span}\left\{V^{\prime}{ }_{1}\right\}$ be left and right deflating subspaces 
of the perturbed matrix pair $(A+E, B+F)$. Then using the technique of Sun [27] one can prove the following first order bounds:

$$
\begin{aligned}
& \sin \theta_{\max }\left(\mathcal{L}, \mathcal{L}^{\prime}\right) \leq\left\|U_{1}-U^{\prime}{ }_{1}\right\|_{F} \leq \frac{\|(E, F)\|_{F}}{\operatorname{Dif}_{l}\left[\left(A_{11}, B_{11}\right),\left(A_{22}, B_{22}\right)\right]}+O\left(\|(E, F)\|_{F}^{2}\right), \\
& \sin \theta_{\max }\left(\mathcal{R}, \mathcal{R}^{\prime}\right) \leq\left\|V_{1}-V^{\prime}{ }_{1}\right\|_{F} \leq \frac{\|(E, F)\|_{F}}{\operatorname{Dif}_{l}\left[\left(A_{11}, B_{11}\right),\left(A_{22}, B_{22}\right)\right]}+O\left(\|(E, F)\|_{F}^{2}\right) .
\end{aligned}
$$

From the series expansion of the arcsine function we can simplify these bounds further, giving the asymptotic angular bounds

$$
\begin{aligned}
\theta_{\max }\left(\mathcal{L}, \mathcal{L}^{\prime}\right) & \lesssim \frac{\|(E, F)\|_{F}}{\operatorname{Dif}_{l}\left[\left(A_{11}, B_{11}\right),\left(A_{22}, B_{22}\right)\right]}, \\
\theta_{\max }\left(\mathcal{R}, \mathcal{R}^{\prime}\right) & \lesssim \frac{\|(E, F)\|_{F}}{\operatorname{Dif}_{l}\left[\left(A_{11}, B_{11}\right),\left(A_{22}, B_{22}\right)\right]} .
\end{aligned}
$$

These bounds imply that $\operatorname{Dif}_{l}$ is the reciprocal of the condition number for eigenvectors $(m=1)$ and deflating subspaces $(m>1)$ of a regular $(A, B)$.

\subsubsection{Upper Bound on Perturbations and Global Error Bounds for Deflating Subspaces and Clustered Eigenvalues}

In order to guarantee that the clusters in the $(1,1)$-blocks of $(A, B)$ and the perturbed matrix pair $(A+E, B+F)$ are of the same size $m$ and uniquely defined, we have to put restrictions on $\|(E, F)\|_{F}[9]$ :

$$
\|(E, F)\|_{F} \leq \frac{\min \left(\operatorname{Dif}_{u}, \operatorname{Dif}_{l}\right)}{\left(p^{2}+q^{2}\right)^{1 / 2}+2 \max (p, q)} .
$$

By imposing a somewhat stronger condition on $\|(E, F)\|_{F}$, namely

$$
\|(E, F)\|_{F} \leq \frac{\min \left(\operatorname{Dif}_{u}, \operatorname{Dif}_{l}\right)}{4 \max (p, q)} \equiv \Delta,
$$

$\Delta$ in (4.21) conform to the corresponding restriction for the standard eigenvalue problem $\left(\operatorname{Sep}\left(A_{11}, A_{22}\right) / 4 p\right)[3]$.

We see that $\Delta$ may be small if the separation between the two matrix pairs is small or the left and right projection norms are large, indicating that the (deflating subspace) problem is ill-conditioned. A larger $\|(E, F)\|_{F}(>\Delta)$ may imply that one eigenvalue in the cluster moves and coalesces with another eigenvalue (outside the cluster). Indeed, $\Delta$ is a lower bound on the smallest $\|(E, F)\|_{F}$ such that an eigenvalue of $\left(A_{11}, B_{11}\right)$ coalesces with an eigenvalue of $\left(A_{22}, B_{22}\right)$ under perturbation $(E, F)$. The bound $\Delta$ can be quite conservative but is almost exact in some cases and a good estimate in many others. In particular, the following global error bounds for a pair of deflating subspaces is guaranteed valid for $\|(E, F)\|_{F} \leq \Delta[9]$.

As before, we assume that $(A, B)$ is in generalized Schur form (4.10) and that $\left(A_{11}, B_{11}\right)$ contains the cluster of $m$ eigenvalues with left and right deflating subspaces $\mathcal{L}$ and $\mathcal{R}$, 
respectively. Further, let $\mathcal{L}^{\prime}$ and $\mathcal{R}^{\prime}$ be left and right deflating subspaces of $(A+E, B+F)$. Then we have the following angle bounds for left and right deflating subspaces of the unperturbed and perturbed matrix pairs [9]:

If $\delta \equiv\|(E, F)\|_{F} / \Delta<1$, then

$$
\begin{gathered}
\theta_{\max }\left(\mathcal{L}, \mathcal{L}^{\prime}\right) \leq \arctan \left(\frac{\delta}{p-\delta\left(p^{2}-1\right)^{1 / 2}}\right) \\
\theta_{\max }\left(\mathcal{R}, \mathcal{R}^{\prime}\right) \leq \arctan \left(\frac{\delta}{q-\delta\left(q^{2}-1\right)^{1 / 2}}\right) .
\end{gathered}
$$

In other words, if $\delta$ is small, then the perturbed pair of left and right deflating subspaces are small perturbations of the exact pair of deflating subspaces.

The bounds (4.22), (4.23) are generalized and extended to pairs of reducing subspaces for singular $(A, B)[9,10,11]$.

We are also interested to bound the error in the average of the eigenvalues of the cluster in $\left(A_{11}, B_{11}\right)$. However, since we are faced with both finite and infinite eigenvalues it is not clear how to define the average of the $m$ eigenvalues $\lambda_{i}=\alpha_{i} / \beta_{i}$. Only if we require that $\left(A_{11}, B_{11}\right)$ contains a (proper) subset of the finite eigenvalues or all infinite eigenvalues (and no finite eigenvalues) does the average of the cluster make sense. In the following theorem we distinguish these two cases and formulate error bounds for the average of the clustered eigenvalues.

Theorem 4.1 Let $\left(A_{11}, B_{11}\right)$ denote the block of the generalized Schur form of $(A, B)$ that correspond to the unperturbed cluster of eigenvalues, with

$$
\operatorname{Dif}_{x}\left[\left(A_{11}, B_{11}\right),\left(A_{22}, B_{22}\right)\right]>0 \text { for } x=l, u \text {. }
$$

Similarly, let $\left(A^{\prime}{ }_{11}, B^{\prime}{ }_{11}\right)$ denote the corresponding block with perturbed eigenvalues associated with $(A+E, B+F)$, where $\|(E, F)\|_{F} \leq \Delta(4.21)$.

Case 1. $\left(A_{11}, B_{11}\right)$ contains a (proper) subset of the finite eigenvalues, i.e. $B_{11}$ is nonsingular. Let $\bar{\lambda}$ denote the average of the $m$ unperturbed eigenvalues of $\left(A_{11}, B_{11}\right)$ and let $\bar{\lambda}^{\prime}$ be the corresponding average of the perturbed eigenvalues of $\left(A^{\prime}{ }_{11}, B^{\prime}{ }_{11}\right)$. Then

$$
\left|\bar{\lambda}-\overline{\lambda^{\prime}}\right| \leq \frac{1}{\sigma_{\min }\left(B_{11}\right)}\left(1+\frac{\sigma_{\max }\left(A^{\prime}{ }_{11}\right)}{\sigma_{\min }\left(B^{\prime}{ }_{11}\right)}\right) 3 p\|(E, F)\|_{F} .
$$

Case 2. $\left(A_{11}, B_{11}\right)$ contains all infinite eigenvalues and no finite eigenvalues, i.e. $A_{11}$ is non-singular. Let $\bar{\mu}=0$ denote the average of the $m$ unperturbed eigenvalues of the reciprocal problem $\left(B_{11}, A_{11}\right)$ and, similarly, let $\bar{\mu}^{\prime}$ be the corresponding average of the perturbed eigenvalues of $\left(B^{\prime}{ }_{11}, A^{\prime}{ }_{11}\right)$. Then

$$
\left|\bar{\mu}-\bar{\mu}^{\prime}\right| \leq \frac{1}{\sigma_{\min }\left(A_{11}\right)}\left(1+\frac{\sigma_{\max }\left(B_{11}^{\prime}\right)}{\sigma_{\min }\left(A_{11}^{\prime}\right)}\right) 3 p\|(E, F)\|_{F} .
$$

Proof In general, we can bound the average $\bar{\lambda}$ of the eigenvalues of an $m \times m$ matrix $C$ as

$$
|\bar{\lambda}| \leq \frac{1}{m} \sum_{i=1}^{m}\left|\lambda_{i}\right| \leq \max _{i}\left|\lambda_{i}\right| \leq\|C\|_{2} \leq\|C\|_{F} .
$$


In case 1 we have

$$
\left|\bar{\lambda}-\overline{\lambda^{\prime}}\right| \leq\left\|B_{11}^{-1} A_{11}-{B^{\prime}}_{11}^{-1} A_{11}^{\prime}\right\|_{F} \leq\left\|B_{11}^{-1}\right\|_{2}\left\|A_{11}-A_{11}^{\prime}\right\|_{F}+\left\|B_{11}^{-1}-B_{11}^{\prime-1}\right\|_{F}\left\|A_{11}^{\prime}\right\|_{2} .
$$

Since $B_{11}^{-1}-B_{11}^{\prime-1}=-B_{11}^{\prime-1}\left(B_{11}-B_{11}^{\prime}\right) B_{11}^{-1}$ we have that

$$
\left|\bar{\lambda}-\bar{\lambda}^{\prime}\right| \leq\left\|B_{11}^{-1}\right\|_{2}\left(\left\|A_{11}-A_{11}^{\prime}\right\|_{F}+\left\|B_{11}-B_{11}^{\prime}\right\|_{F}\left\|B_{11}^{\prime-1}\right\|_{2}\left\|A_{11}^{\prime}\right\|_{2}\right) .
$$

With the assumptions in the theorem we can apply the technique of Stewart [25] and prove that

$$
\left\|A_{11}-A_{11}^{\prime}\right\|_{F} \leq 3 p\|(E, F)\|_{F}, \quad\left\|B_{11}-B^{\prime}{ }_{11}\right\|_{F} \leq 3 p\|(E, F)\|_{F},
$$

where $p(4.14)$ is the norm of the left spectral projector.

Using these bounds in (4.26) give us the error bound (4.24).

In case 2 we have

$$
\left|\bar{\mu}-\bar{\mu}^{\prime}\right| \leq\left\|A_{11}^{-1} B_{11}-A_{11}^{\prime-1} B_{11}^{\prime}\right\|_{F},
$$

and the error bound (4.25) can be proved similarly.

In the following we make some comments to the error bounds in Theorem 4.1. If $B_{11}$ (in case 1 ) and $A_{11}$ (in case 2 ) are well-conditioned with respect to inversion (i.e. $\sigma_{\min }\left(B_{11}\right) \gg 0$ and $\sigma_{\min }\left(A_{11}\right) \gg 0$, respectively) then the error bounds can be expressed as

$$
\left|\bar{\lambda}-\overline{\lambda^{\prime}}\right| \leq c_{1} p\|(E, F)\|_{F}, \quad\left|\bar{\mu}-\overline{\mu^{\prime}}\right| \leq c_{2} p\|(E, F)\|_{F},
$$

where $c_{1}$ and $c_{2}$ are modest constants. These bounds conform with the corresponding error bound for the average of a cluster of eigenvalues to the standard eigenvalue problem $\left(2 p\|E\|_{F}\right)$. However, if $\sigma_{\min }\left(B_{11}\right)$ (and $\sigma_{\min }\left(B^{\prime}{ }_{11}\right)$ ) are small in case 1 , or if $\sigma_{\min }\left(A_{11}\right)$ (and $\left.\sigma_{\min }\left(A^{\prime}{ }_{11}\right)\right)$ are small in case 2 , then the average of the clustered eigenvalues can be quite sensitive to perturbations in $(A, B)$, which is signaled by the quantities

$$
\frac{1}{\sigma_{\min }\left(B_{11}\right)}\left(1+\frac{\sigma_{\max }\left(A^{\prime}{ }_{11}\right)}{\sigma_{\min }\left(B^{\prime}{ }_{11}\right)}\right),
$$

and

$$
\frac{1}{\sigma_{\min }\left(A_{11}\right)}\left(1+\frac{\sigma_{\max }\left(B_{11}^{\prime}\right)}{\sigma_{\min }\left(A^{\prime}{ }_{11}\right)}\right),
$$

respectively, in the bounds (4.24) and (4.25). In case 1 this means that $\left(A_{11}, B_{11}\right)$ is nearby a pencil with an infinite eigenvalue and in case $2\left(A_{11}, B_{11}\right)$ is nearby a singular pencil. Both cases represent ill-conditioned clustering problems. One way to tackle the most illconditioned cases with multiple infinite eigenvalues and an almost singular $A_{11}$ is to compute and separate the Jordan structure of the infinite eigenvalue before any clustering takes place $[10,11]$. It is well-known that the $Q Z$ algorithm applied to defective infinite eigenvalues can affect otherwise well-conditioned eigenvalues of $(A, B)$ [32]. By separating the infinite structure from the rest of the spectrum before applying the $Q Z$ algorithm we circumvent this problem. 


\subsubsection{Optimal Backward Perturbation of Approximate Left and Right Deflat- ing Subspaces}

Suppose that $\overline{\mathcal{L}}=\operatorname{span}\left(\bar{V}_{1}\right)$ and $\overline{\mathcal{R}}=\operatorname{span}\left(\bar{U}_{1}\right)$, with $\bar{U}_{1}{ }^{H} \bar{U}_{1}=\bar{V}_{1}{ }^{H} \bar{V}_{1}=I_{m}$ are approximate left and right deflating subspaces of $(A, B)$. We are interested to find (backward) perturbations of $(A, B)$ such that the perturbed matrix pair has $\overline{\mathcal{L}}$ and $\overline{\mathcal{R}}$ as exact left and right deflating subspaces. Let

$$
\mathcal{H} \equiv\left\{H=(E, F), \quad E, F \in \mathbf{C}^{n \times n}:(A+E) \overline{\mathcal{R}} \subseteq \overline{\mathcal{L}},(B+F) \overline{\mathcal{R}} \subseteq \overline{\mathcal{L}}\right\}
$$

i.e. $\mathcal{H}$ defines the set of perturbations $H=(E, F)$ such that $(A+E, B+F)$ has $\overline{\mathcal{L}}$ and $\overline{\mathcal{R}}$ as exact left and right deflating subspaces. Moreover, let the right residuals with respect to the approximate deflating subspaces be

$$
R_{\text {res }} \equiv\left(R_{\text {Ares }}, R_{\text {Bres }}\right)=\left(A \bar{U}_{1}-\bar{V}_{1} \bar{A}_{11}, B \bar{U}_{1}-\bar{V}_{1} \bar{B}_{11}\right),
$$

where $\bar{A}_{11}={\overline{V_{1}}}^{H} A \bar{U}_{1}$ and $\bar{B}_{11}=\bar{V}_{1}^{H} B \bar{U}_{1}$. Then there exist a unique optimal backward perturbation $H_{\mathrm{opt}}=-R_{\mathrm{res}} \bar{U}_{1}^{H} \in \mathcal{H}[28]$ :

$$
\left\|H_{\mathrm{opt}}\right\|=\min _{H \in \mathcal{H}}\|H\|=\left\|R_{\mathrm{res}}\right\|,
$$

for any unitarily invariant norm.

\subsubsection{Residual-based Error Bound for Approximate Left and Right Deflating Subspaces}

Assume that $\bar{U}=\left[\bar{U}_{1}, \bar{U}_{2}\right]$ and $\bar{V}=\left[\bar{V}_{1}, \bar{V}_{2}\right]$ are computed transformations that take $(A, B)$ to generalized Schur form:

$$
\bar{V}^{H} A \bar{U}=\left[\begin{array}{cc}
\bar{A}_{11} & \bar{A}_{12} \\
\bar{A}_{21} & \bar{A}_{22}
\end{array}\right], \quad \bar{V}^{H} B \bar{U}=\left[\begin{array}{cc}
\bar{B}_{11} & \bar{B}_{12} \\
\bar{B}_{21} & \bar{B}_{22}
\end{array}\right],
$$

where the entries of $\bar{A}_{21}$ and $\bar{B}_{21}$ are small and $\bar{U}, \bar{V}$ unitary (orthogonal) to machine precision accuracy.

Then $\overline{\mathcal{L}}=\operatorname{span}\left(\bar{V}_{1}\right)$ and $\overline{\mathcal{R}}=\operatorname{span}\left(\bar{U}_{1}\right)$ are approximate left and right deflating subspaces of $(A, B)$ and we compute their left and right residuals:

$$
\begin{gathered}
L_{\mathrm{res}} \equiv\left(L_{\text {Ares }}, L_{\mathrm{Bres}}\right)=\left(\bar{V}_{1}^{H} A-\bar{A}_{11} \bar{U}_{1}^{H}, \bar{V}_{1}^{H} B-\bar{B}_{11} \bar{U}_{1}^{H}\right), \\
R_{\mathrm{res}} \equiv\left(R_{\text {Ares }}, R_{\mathrm{Bres}}\right)=\left(A \bar{U}_{1}-\bar{V}_{1} \bar{A}_{11}, B \bar{U}_{1}-\bar{V}_{1} \bar{B}_{11}\right) .
\end{gathered}
$$

It is straightforward to show that

$$
\left\|L_{\text {Ares }}\right\|_{F}=\left\|\bar{A}_{12}\right\|_{F}, \quad\left\|L_{\text {Bres }}\right\|_{F}=\left\|\bar{B}_{12}\right\|_{F}, \quad\left\|R_{\text {Ares }}\right\|_{F}=\left\|\bar{A}_{21}\right\|_{F}, \quad\left\|R_{\text {Bres }}\right\|_{F}=\left\|\bar{B}_{21}\right\|_{F} .
$$

We see that the norm of the right residuals are always small, while in general we cannot expect the norm of the left residuals to be small. However, knowing that there exist an optimal backward perturbation of approximate left and right deflating subspaces it is possible 
to derive a residual-based error bound. We can rewrite the residual-based error bound for deflating subspaces in [28] as the following angular error bounds:

If $\eta \equiv 4\left\|L_{\text {res }}\right\|_{F}\left\|R_{\text {res }}\right\|_{F} / \operatorname{Dif}_{l}^{2}<1$, then

$$
\begin{gathered}
\theta_{\max }(\mathcal{L}, \overline{\mathcal{L}}) \leq \arctan \left(\frac{2\left\|R_{\text {res }}\right\|_{F}}{\operatorname{Dif}_{l}}\right), \\
\theta_{\max }(\mathcal{R}, \overline{\mathcal{R}}) \leq \arctan \left(\frac{2\left\|R_{\mathrm{res}}\right\|_{F}}{\operatorname{Dif}_{l}}\right) .
\end{gathered}
$$

Notice that the bounds (4.29) and (4.30) are approximate in the sense that the theory assumes that $\bar{U}, \bar{V}$ are exactly unitary (orthogonal), while we can only guarantee that they are unitary (orthogonal) to machine precision accuracy.

From the definition of $\eta$ we also get a bound on $\|(E, F)\|_{F}$ similar to (4.21) that guarantees that the residual-based bounds are valid for perturbations $(E, F)$ fulfilling

$$
\|(E, F)\|_{F} \equiv\left\|R_{\mathrm{res}}\right\|_{F} \leq \frac{\operatorname{Dif}_{l}^{2}}{4\left\|L_{\mathrm{res}}\right\|_{F}} \equiv \Delta_{r}
$$

\subsection{Summary of Error Bounds for Eigenvalues and Eigenspaces}

In Table 4.1 and Table 4.2 we summarize the error bounds presented in Section 4 (see earlier subsections for definitions and notation used). Table 4.1 shows the asymptotic bounds for a simple eigenvalue $(\alpha, \beta)$ where $\lambda=\alpha / \beta$, the average of a cluster of eigenvalues $\bar{\lambda}$ (or $\bar{\mu}=0$ for the infinite eigenvalues, i.e. $\bar{\lambda}=1 / \bar{\mu})$, a left and right eigenvector pair $y$ and $x$, and a pair (left and right) of deflating subspaces $\mathcal{L}$ and $\mathcal{R}$.

Table 4.1: Asymptotic error bounds for the generalized eigenvalue problem

\begin{tabular}{|l|c|c|}
\hline Bounds for & Error bound & Comment \\
\hline Simple eigenvalue: & $\mathcal{X}\left((\alpha, \beta),\left(\alpha^{\prime}, \beta^{\prime}\right)\right) \lesssim \nu\|(E, F)\|_{F}$ & $\lambda=\alpha / \beta$ \\
\hline Eigenvalue cluster: & $\mid \bar{\lambda}-\overline{\lambda^{\prime}} \lesssim c_{1} p\|(E, F)\|_{F}$ & See also global bounds \\
Average of finite $\lambda_{i}$ & $\mid \bar{\mu}-\bar{\mu}^{\prime} \lesssim c_{2} p\|(E, F)\|_{F}$ & $\left(c_{1}\right.$ and $c_{2}$ are constants $)$ \\
Average of infinite $\lambda_{i}=1 / \mu_{i}$ & & \\
\hline Eigenvector pair: & $\theta_{\max }\left(y, y^{\prime}\right) \lesssim\|(E, F)\|_{F} /$ Dif $_{l}$ & $m=1$ \\
Left & $\theta_{\max }\left(x, x^{\prime}\right) \lesssim\|(E, F)\|_{F} /$ Dif $_{l}$ & $m=1$ \\
Right & $\theta_{\max }\left(\mathcal{L}, \mathcal{L}^{\prime}\right) \lesssim\|(E, F)\|_{F} / \operatorname{Dif}_{l}$ & $1<m \leq n-1$ \\
\hline Deflating subspace pair: & $\theta_{\max }\left(\mathcal{R}, \mathcal{R}^{\prime}\right) \lesssim\|(E, F)\|_{F} /$ Dif $_{l}$ & $1<m \leq n-1$ \\
Left &
\end{tabular}

The asymptotic bounds are only valid for sufficiently small perturbations. If the problem is ill-conditioned, the asymptotic bounds may only hold for extremely small values of $\|(E, F)\|$. Therefore, we also provide similar global error bounds (displayed in Table 
4.2), which are valid for all perturbations that satisfy an upper bound on $\|(E, F)\|$. These restrictions are $\Delta(4.21)$ and $\Delta_{r}(4.31)$, where $\Delta_{r}$ is associated with residual-based error bounds. For ill-conditioned problems these restrictions will also be small. Indeed, a small value of $\Delta$ (or $\Delta_{r}$ ) shows that the cluster of eigenvalues in the leading $m \times m$ blocks of $(A, B)$ is ill-conditioned in the sense that small perturbations of $(A, B)$ may imply that one eigenvalue in the cluster moves and coalesces with another eigenvalue (outside the cluster). Accordingly, this also means that the associated (left and right) deflating subspaces are sensitive for small perturbations, since the size of the perturbed subspaces may change for small perturbations of $(A, B)$.

Table 4.2: Global error bounds for the generalized eigenvalue problem

\begin{tabular}{|c|c|c|}
\hline Bounds for & Error bound & Restriction on $\|(E, F)\|_{F}$ \\
\hline Simple eigenvalue: & Bound (4.8) & None (holds for all $(E, F)$ ) \\
\hline$\left(\lambda=\alpha / \beta,|\alpha|^{2}+|\beta|^{2}=1\right)$ & Bound (4.9) & None (holds for all $(E, F)$ ) \\
\hline Eigenvalue cluster: & & \\
\hline Average of finite $\lambda_{i}$ & Bound (4.24) & $\leq \Delta \equiv \min \left(\operatorname{Dif}_{u}, \operatorname{Dif}_{l}\right) / 4 \max (p, q)$ \\
\hline Average of infinite $\lambda_{i}=1 / \mu_{i}$ & Bound (4.25) & $\leq \Delta \equiv \min \left(\operatorname{Dif}_{u}, \operatorname{Dif}_{l}\right) / 4 \max (p, q)$ \\
\hline Eigenvector pair: & & \\
\hline Left & Bound (4.22) & $\leq \Delta \equiv \min \left(\operatorname{Dif}_{u}, \operatorname{Dif}_{l}\right) / 4 \max (p, q)$ \\
\hline Right & Bound (4.23) & $\leq \Delta \equiv \min \left(\operatorname{Dif}_{u}, \operatorname{Dif}_{l}\right) / 4 \max (p, q)$ \\
\hline Left (residual-based) & Bound (4.29) & $\leq \Delta_{r} \equiv \operatorname{Dif}_{l}^{2} / 4\left\|L_{\mathrm{res}}\right\|_{F}$ \\
\hline Right (residual-based) & Bound (4.30) & $\leq \Delta_{r} \equiv \operatorname{Dif}_{l}^{2} / 4\left\|L_{\mathrm{res}}\right\|_{F}$ \\
\hline Deflating subspace pair: & & \\
\hline Left & Bound (4.22) & $\leq \Delta \equiv \min \left(\operatorname{Dif}_{u}, \operatorname{Dif}_{l}\right) / 4 \max (p, q)$ \\
\hline Right & Bound (4.23) & $\leq \Delta \equiv \min \left(\operatorname{Dif}_{u}, \operatorname{Dif}_{l}\right) / 4 \max (p, q)$ \\
\hline Left (residual-based) & Bound (4.29) & $\leq \Delta_{r} \equiv \operatorname{Dif}_{l}^{2} / 4\left\|L_{\mathrm{res}}\right\|_{F}$ \\
\hline Right (residual-based) & Bound (4.30) & $\leq \Delta_{r} \equiv \operatorname{Dif}_{l}^{2} / 4\left\|L_{\mathrm{res}}\right\|_{F}$ \\
\hline
\end{tabular}

It is interesting to compare the sizes of $\Delta$ and $\Delta_{r}$. We focus on ill-conditioned problems where the separation between the two clusters are small (i.e. both $\operatorname{Dif}_{l}$ and $\operatorname{Dif}_{u}$ are small) and the deflating subspaces are sensitive (i.e. the associated generalized Sylvester equation has large-normed solutions $(L, R))$. Since Dif ${ }_{l}^{2}$ appears in the nominator of $\Delta_{r}$ while we only have $\min \left(\operatorname{Dif}_{u}, \operatorname{Dif}_{l}\right)$ in the nominator of $\Delta$ it seems as if $\Delta_{r}$ puts harder restrictions on the perturbations. However, if we use the expressions (4.28) in $\Delta_{r}$, the bound (4.17) on $(L, R)$ to bound $p$ and $q$ giving that they are of size $O\left(\left\|\left(\bar{A}_{12}, \bar{B}_{12}\right)\right\|_{F} / \mathrm{Dif}_{u}\right)$, then we see that $\Delta$ and $\Delta_{r}$ are qualitatively of the same size. It is of course possible to construct examples where $\Delta$ is smaller than $\Delta_{r}$ and vice versa.

\subsection{Condition Estimates and Error Bounds Computed}

Our software (described in more detail in Section 5) compute estimates of the following quantities that appear in the condition numbers and error bounds summarized in tables 4.1 and 4.2 . 
- $S(\lambda)=\nu^{-1}$, the reciprocal value of the condition number $\nu$ (4.4) for an individual eigenvalue $\lambda=\alpha / \beta$.

Given the left and right eigenvectors $y$ and $x$ corresponding to $\lambda$, the reciprocal condition number is computed in $O\left(n^{2}\right)$ flops (floating point operations) as

$$
S(\lambda)=\frac{\sqrt{\left|y^{H} A x\right|^{2}+\left|y^{H} B x\right|^{2}}}{\|x\|_{2}\|y\|_{2}} .
$$

If both $\alpha$ and $\beta$ are zero, then $(A, B)$ is singular and $S(\lambda)=-1$ is reported.

The (left and right) eigenvectors computed by LAPACK are normalized such that the largest component will have the sum of the modulus of the real and imaginary parts equal to one (e.g., see _GEGV).

- $\operatorname{Dif}_{u}$ (4.16) and $\operatorname{Dif}_{l}$ (4.18), i.e. the separation(s) between two matrix pairs.

Dif $_{l}$ is a reciprocal condition number for an individual (left or right) eigenvector or a (left or right) deflating subspace (see Section 4.2.2). Both Dif ${ }_{u}$ and Dif ${ }_{l}$ appear in $\Delta$. Our algorithms for estimating $\operatorname{Dif}_{u}$ and $\operatorname{Dif}_{l}$ are discussed in Section 4.4.1.

- $p^{-1}$ and $q^{-1}$, the reciprocal values of the left and right projector norms as defined in (4.14).

These values are computed straightforwardly using $L$ and $R$ from the generalized Sylvester equation (4.12). The cost for solving (4.12) is $2 m^{2}(n-m)+2 m(n-m)^{2}$ flops, where $m \geq 1$ is the dimension of the deflating subspace(s) corresponding to the selected eigenvalues. Given $L$ and $R$ the cost for computing $p^{-1}$ and $q^{-1}$ is only $O(m(n-m))$ flops.

By using the estimates of these quantities in the error bounds, the user gets enough information for assessing the accuracy of computed eigenvalues (or the average of clustered eigenvalues), eigenvectors or deflating subspaces.

We also compute the algorithm-independent residual-based error bound(s) (4.29), (4.30) and $\eta$, the condition that guarantees the validity of the bound(s). The residual-based error bound and $\eta$ are computed using a Frobenius normed-based estimate of $\operatorname{Dif}_{l}$ (see Section 4.4.1). The residuals (4.28) associated with the approximate deflating subspaces are computed straightforwardly.

\subsubsection{Estimating $\operatorname{Dif}_{u}$ and $\operatorname{Dif}_{l}$}

LAPACK-style algorithms and software for estimating $\operatorname{Dif}_{u}$ (4.16) are presented in [19]. The basic problem is to find a lower bound on $\operatorname{Dif}_{u}^{-1}\left[\left(A_{11}, B_{11}\right),\left(A_{22}, B_{22}\right)\right] \equiv\left\|Z_{u}^{-1}\right\|_{2}$, where $Z_{u}$ is the matrix representation (4.13) of the generalized Sylvester operator. It is possible to compute lower bounds on Dif $f_{u}^{-1}$ by solving generalized Sylvester equations in triangular form. Both Frobenius norm-based and one-norm-based Dif ${ }_{u}$-estimators are discussed and evaluated in [19]. The one-norm-based estimator makes the condition estimation uniform with other parts of LAPACK (e.g., the standard eigenvalue problem). The Frobenius normbased estimator offers a low-cost and equally reliable estimator. The one-norm-based estimator is a factor 3-10 times more expensive. 
By knowing a lower bound DIFINV on $\left\|Z_{u}^{-1}\right\|_{2}$ we also have an upper bound DIF = $1 /$ DIFINV on the separation between two regular matrix pairs. Since we use blocked algorithms to solve the generalized Sylvester equations involved in computing DIFINV, our estimators will mainly execute Level 3 operations. In the following we outline the algorithms for the $\operatorname{Dif}_{u}$-estimators. From the definition of $\operatorname{Dif}_{l}$ (4.18) we see that Dif ${ }_{l}$-estimators can be computed by using our algorithms for estimating Dif ${ }_{u}$. Our software provide (optionally) both Frobenius norm-based and one-normed-based estimators for $\operatorname{Dif}_{u}$ and $\operatorname{Dif}_{l}$, respectively ( see Section 5).

A Frobenius Norm-Based Estimator From the $Z_{u} x=b$ representation (4.13) of the generalized Sylvester equation (4.12) we get a lower bound on $\operatorname{Dif}_{u}^{-1}$ :

$$
\|(L, R)\|_{F} /\|(C, F)\|_{F}=\|x\|_{2} /\|b\|_{2} \leq\left\|Z_{u}^{-1}\right\|_{2} .
$$

To get an improved estimate we want to choose right hand sides $\left(C^{*}, F^{*}\right)$ such that the associated solution $\left(L^{*}, R^{*}\right)$ has as large norm as possible. Then the quantity

$$
\phi_{F} \equiv\left\|\left(L^{*}, R^{*}\right)\right\|_{F} /\left\|\left(C^{*}, F^{*}\right)\right\|_{F},
$$

is our lower bound on $\left\|Z_{u}^{-1}\right\|_{2}$. The work to compute $\phi_{F}$ is comparable to solve a generalized Sylvester equation, which costs $O\left(m^{3}+m^{2}(n-m)+m(n-m)^{2}+(n-m)^{3}\right)$ flops (only $2 m^{2}(n-m)+2 m(n-m)^{2}$ if the matrix pairs are in generalized Schur form) [21]. This is a very modest cost compared to compute the exact value of $\sigma_{\min }\left(Z_{u}\right)$, which requires $O\left(m^{3}(n-m)^{3}\right)$ flops.

Two Frobenius norm-based estimators _TDIFE and _TDIFD are discussed in [19], which are modifications of estimators BSOLVE and BSOLVD in [21]. The main differences concern how contributions to $\phi_{F}$ from different subsystems are computed and the look ahead strategies of the estimators. _TDIFE is the default Frobenius norm-based estimator in our software (see Section 5).

An One-Norm-Based Estimator From the relationship

$$
\frac{1}{\sqrt{2 m(n-m)}}\left\|Z_{u}^{-1}\right\|_{1} \leq\left\|Z_{u}^{-1}\right\|_{2} \leq \sqrt{2 m(n-m)}\left\|Z_{u}^{-1}\right\|_{1}
$$

we know that $\left\|Z_{u}^{-1}\right\|_{1}$ can never differ more than a factor $\sqrt{2 m(n-m)}$ from $\left\|Z_{u}^{-1}\right\|_{2}$. So it makes sense to compute an one-norm-based estimator of Dif ${ }_{u}^{-1}$.

The LAPACK routine _LACON implements a method for estimating the one-norm of a square matrix, using reverse communication for evaluating matrix-vector products [14, 15$]$. We apply this method to $\left\|Z_{u}^{-1}\right\|_{1}$ by providing the solution vectors $x$ and $y$ of $Z_{u} x=z$ and a transposed system $Z_{u}^{T} y=z$, where $z$ is determined by LACON. In each step only one of these generalized Sylvester equations is solved using blocked algorithms [19]. The cost for computing this bound is roughly equal to the number of steps in the reverse communication times the cost for one generalized Sylvester solve.

Notice, $\left\|Z_{u}^{-1}\right\|_{\infty}$ also satisfy (4.35), i.e. can never differ more than a factor $\sqrt{2 m(n-m)}$ from $\left\|Z_{u}^{-1}\right\|_{2}$. Moreover, since $\|B\|_{\infty}=\left\|B^{T}\right\|_{1}$ the same method can be used to compute an infinity-norm-based estimate of $\operatorname{Dif}_{u}^{-1}$. 


\subsubsection{Estimating Dif for Individual Eigenvectors Associated with a Complex Conjugate Pair of Eigenvalues}

The estimation of $\operatorname{Dif}_{l}$ discussed in the preceding subsection is applicable to pairs of deflating subspaces $(m \geq 2)$ as well as to individual eigenvector pairs $(m=1)$. As before we assume that $(A, B)$ is transformed to generalized Schur form

$$
Q^{H} A Z=S \equiv\left[\begin{array}{cc}
S_{11} & S_{12} \\
0 & S_{22}
\end{array}\right], \quad Q^{H} B Z=T \equiv\left[\begin{array}{cc}
T_{11} & T_{12} \\
0 & T_{22}
\end{array}\right] .
$$

In complex arithmetic we can always choose $\left(S_{11}, T_{11}\right)$ to be the individual eigenvalue $\lambda_{1}=$ $\alpha_{1} / \beta_{1}=S_{11} / T_{11}$ (real or complex) we want to consider. Moreover, the first column of $Q$ and $Z$ form a pair of deflating subspaces, where $q_{1}$ also is a right eigenvector corresponding to $\lambda_{1}$. In this case we can, for example, apply the one-normed-based estimator to estimate $\operatorname{Dif}_{l}$.

What is said above also applies in real arithmetic to real eigenvalues of $(A, B)$. However, there is an extra complication to estimate Dif for the individual eigenvectors corresponding to a complex conjugate pair of eigenvalues. For a real matrix pair, $Q$ and $Z$ in (4.36) are orthogonal and $\left(S_{11}, T_{11}\right)$ is a $2 \times 2$ matrix pair corresponding to the complex conjugate pair of eigenvalues $\lambda_{1}$ and $\bar{\lambda}_{1}$. It exits unitary $U_{1}$ and $V_{1}$ such that

$$
U_{1}^{H} S_{11} V_{1}=\left[\begin{array}{cc}
\alpha_{1} & x \\
0 & \alpha_{2}
\end{array}\right], \quad U_{1}^{H} T_{11} V_{1}=\left[\begin{array}{cc}
\beta_{1} & y \\
0 & \beta_{2}
\end{array}\right]
$$

where $\alpha_{1}=\gamma_{1}+i \mu_{1}, \alpha_{2}=\gamma_{2}+i \mu_{2}$ and $\beta_{1}, \beta_{2}$ are real numbers. Now, the complex conjugate pair of eigenvalues is given by $\lambda_{1}=\alpha_{1} / \beta_{1}$ and $\bar{\lambda}_{1}=\alpha_{2} / \beta_{2}$ (i.e. $\gamma_{1} / \beta_{1}=\gamma_{2} / \beta_{2}$ and $\left.\mu_{1} / \beta_{1}=\mu_{2} / \beta_{2}\right)$.

If we are interested to estimate $\operatorname{Dif}_{l}$ associated with $\lambda_{1}$, then we can choose

$$
U=\left[\begin{array}{cc}
U_{1} & 0 \\
0 & I_{n-2}
\end{array}\right], \quad V=\left[\begin{array}{cc}
V_{1} & 0 \\
0 & I_{n-2}
\end{array}\right],
$$

and we get

$$
U^{H} S V=\left[\begin{array}{cc}
\alpha_{1} & S^{\prime}{ }_{12} \\
0 & S_{22}^{\prime}
\end{array}\right], \quad U^{H} T V=\left[\begin{array}{cc}
\beta_{1} & T_{12}^{\prime} \\
0 & T_{22}^{\prime}
\end{array}\right] .
$$

Notice that only $\left(S^{\prime}{ }_{12}, T^{\prime}{ }_{12}\right)$ and the first row of $\left(S^{\prime}{ }_{22}, T^{\prime}{ }_{22}\right)$ have complex entries. Moreover, $\bar{\lambda}_{1}=\alpha_{2} / \beta_{2}$ belongs to the spectrum of $\left(S_{22}^{\prime}, T_{22}^{\prime}\right)$.

From [9] we have the following explicit expression for Dif : $_{\text {: }}$

$$
\operatorname{Dif}_{l}\left[\left(\alpha_{1}, \beta_{1}\right),\left(S_{22}^{\prime}, T_{22}^{\prime}\right)\right]=\sigma_{\min }\left(Z_{l}\right),
$$

where

$$
Z_{l}=\left[\begin{array}{cc}
\alpha_{1} \otimes I_{n-1} & -1 \otimes S_{22}^{\prime} \\
\beta_{1} \otimes I_{n-1} & -1 \otimes T_{22}^{\prime}
\end{array}\right]
$$

and $Z_{l}$ is a $2(n-1) \times 2(n-1)$ matrix. Dif $l$ associated with $\left(\alpha_{1}, \beta_{1}\right)$ and its conjugate $\left(\alpha_{2}, \beta_{2}\right)$ have the same value. 
In contrary to the standard eigenvalue problem [3], there is no simple and inexpensive trick to stay in real arithmetic and perform a one-normed-based estimate of $\left\|Z_{l}^{-1}\right\|_{2}$. The $L U$ factorization of $Z_{l}$ may give $L$ and $U$ with all entries having non-zero imaginary parts. Accordingly, the cost for doing the estimation of $\mathrm{Dif}_{l}$ (in real arithmetic) is similar to the cost for doing it entirely in complex arithmetic. From the definition of $\mathrm{Dif}_{l}$ it is possible to show the following two inequalities:

$$
\sigma_{\min }\left(Z_{l}\right) \leq \operatorname{Dif}_{l}\left[\left(\alpha_{1}, \beta_{1}\right),\left(\alpha_{2}, \beta_{2}\right)\right]=\sigma_{\min }\left(\left[\begin{array}{cc}
\alpha_{1} & -\alpha_{2} \\
\beta_{1} & -\beta_{2}
\end{array}\right]\right) \equiv d_{1},
$$

and

$$
\sigma_{\min }\left(Z_{l}\right) \leq \max \left(1,\left|\frac{\gamma_{1}}{\gamma_{2}}\right|\right) \operatorname{Dif}_{l}\left[\left(S_{11}, T_{11}\right),\left(S_{22}, T_{22}\right)\right] \equiv d_{2},
$$

where the $S_{i i}$ and $T_{i i}$ blocks are from the generalized real Schur form. Now, we choose $\min \left(d_{1}, d_{2}\right)$ as our estimate for $\operatorname{Dif}_{l}=\sigma_{\min }\left(Z_{l}\right)$. This estimate can be weaker than the estimate computed in complex arithmetic, but is normally a sufficiently good estimate. We report results for both the real and complex estimators in Section 6.1.3 for a selection of problems. If $\lambda_{1}$ and $\bar{\lambda}_{1}$ are close but well-separated from the rest of the spectrum, then $d_{1}$ is a good estimate of $\sigma_{\min }\left(Z_{l}\right)$. Whether $d_{2}$ is a good estimate to $\sigma_{\min }\left(Z_{l}\right)$ will mainly depend on the size of $\left(S^{\prime}{ }_{12}, T^{\prime}{ }_{12}\right)$ in (4.38) (i.e. the "departure from block-diagonality" of the generalized Schur form).

\section{Outline of the Software}

Following the LAPACK conventions and standards [1], we have developed Fortran 77 routines that perform the following computations for a regular matrix pair $(A, B)$ (in generalized Schur form):

- reorder eigenvalues (diagonal blocks) in the generalized Schur form (routines _TGEXC and_TGEX2),

- compute (left and right) deflating subspaces with specified eigenvalues (routine _TGSEN),

- estimate condition numbers for specified eigenvalues (or a cluster of eigenvalues) and associated eigenvectors or deflating subspaces (routines _TGSNA, _TGSEN), and compute residual-based approximate error bounds for a pair of deflating subspaces (routine _GSRBB).

Following the LAPACK conventions for naming, _ in _YYZZZ stands for S(ingle), D(ouble), $\mathrm{C}$ (omplex) or $\mathrm{Z}$ (Double complex). Routines for all four data types are available. In the following, we describe these top-level computational routines in some detail, while the auxiliary routines are just mentioned briefly. The software uses LAPACK routines to compute machine dependent thresholds, generalized Schur forms of matrix pairs, eigenvalues and eigenvectors, matrix factorizations $(Q R$ and $R Q)$, matrix norms, and to copy matrices, perform column- and row-swapping and so on. BLAS routines are used to perform basic linear algebra operations such as matrix-matrix (Level 3), matrix-vector (Level 2) and vector (Level 1) operations. 


\subsection{Reordering of Diagonal Blocks}

_TGEXC reorders the diagonal blocks of $(A, B)$ in generalized Schur form. The reordering is specified by the parameters IFST and ILST. The diagonal block with row index IFST is moved to row ILST by a sequence of transpositions of adjacent blocks. If IFST (on entry) pointed to the second row of a $2 \times 2$ block, it is changed to point to the first row. ILST always points to the first row of the block in its final position, which may differ from its input value by +1 or -1 . Each swap in the reordering is performed with a call to _TGEX2 (see below), and optionally, the matrices of generalized Schur vectors $Q$ and $Z$ are updated with the orthogonal (unitary) equivalence transformations performed. INFO reports if the reordering was successful or if any swap was rejected due to ill-conditioning. The calling sequence and the leading comment lines of DTGEXC are listed in Appendix A.

_TGEX2 implements a direct algorithm with guaranteed backward stability for swapping two adjacent diagonal blocks $\left(A_{11}, B_{11}\right)$ and $\left(A_{22}, B_{22}\right)$ of a matrix pair $(A, B)$ in generalized Schur form, where the diagonal blocks are of size $\mathbb{N} 1 \times \mathbb{N} 1$ and $\mathbb{N} 2 \times \mathbb{N} 2$, respectively. In the real case $\mathrm{N} 1$ and $\mathrm{N} 2$ are 1 or 2 . If at least one of them is 2, method 2 in Section 3.2 is used to perform the swapping and stability tests. If both eigenvalues are real, $\mathbb{N} 1=\mathrm{N} 2=1$ and the swapping is performed using (orthogonal) Givens rotations [30]. In the complex case we perform all reordering with (unitary) Givens rotations. If the problem is too illconditioned (i.e. the swap does not pass the stability tests), the swap is rejected. As for -TGEXC, this information is reported on exit by the parameter INFO. The user specifies the index of the first block $\left(A_{11}, B_{11}\right)$ in parameter J1. Optionally, the routine also accumulates the orthogonal (unitary) equivalence transformation in $Q$ and $Z$. The calling sequence and the leading comment lines of DTGEX2 are listed in Appendix B.

\subsection{Computing Deflating Subspaces with Specified Eigenvalues}

_TGSEN computes (left and right) deflating subspaces associated with some specified eigenvalues of $(A, B)$ in generalized Schur form. Using _TGEXC, $(A, B)$ is reordered so that a selected cluster of $\mathrm{M}$ eigenvalues appears in the leading $\mathrm{M} \times \mathrm{M}$ diagonal blocks of $A$ and $B$. The logical array SELECT specifies the eigenvalues in the cluster, and thereby the value of M. In the real case, if an eigenvalue that belongs to a complex conjugate pair is selected, then by default its conjugate will also belong to the selected cluster (which conforms to the standard eigenvalue problem in LAPACK). Optionally, the matrices of generalized Schur vectors $Q$ and $Z$ are updated with the orthogonal (unitary) equivalence transformations performed. Then, the leading M columns of $Q$ and $Z$ form orthonormal (unitary) bases of the associated left and right deflating subspaces. By calling the LAPACK routine _GEGV, _TGSEN also computes the generalized eigenvalue pairs $\left(\alpha_{i}, \beta_{i}\right)$ for $i=1: n$, where $\alpha_{i}$ is a complex number and $\beta_{i}$ is a real number. In the real case, real and imaginary parts of $\alpha_{i}$ are reported in $\operatorname{ALPHAR}(i)$ and $\operatorname{ALPHAI}(i)$, respectively, and $\beta_{i}$ in BETA $(i)$. In the complex case, they are reported in the complex arrays ALPHA and BETA. INFO reports if the reordering was

successful or if any swap was rejected due to ill-conditioning. The calling sequence and the leading comment lines of DTGSEN are listed in Appendix C. 


\subsection{Condition Estimation and Approximate Error Bounds}

Optionally, _TGSEN also computes estimates of quantities (condition numbers) that appear in the error bounds summarized in tables 4.1 and 4.2. These are the reciprocal values of the left and right projection norms $p$ and $q$ (defined in (4.14)), and estimates of the separation between two matrix pairs defined by $\operatorname{Dif}_{u}\left[\left(A_{11}, B_{11}\right),\left(A_{22}, B_{22}\right)\right](4.16)$ and $\operatorname{Dif}_{l}\left[\left(A_{11}, B_{11}\right),\left(A_{22}, B_{22}\right)\right]$ (4.17). The reciprocal values of $p$ and $q$ are reported in PL and PR, respectively, and estimates of Dif $u$ and $\operatorname{Dif}_{l}$ in DIF(1) and DIF(2), respectively. The functionality obtained from _TGSEN is specified by setting the parameter IJOB,

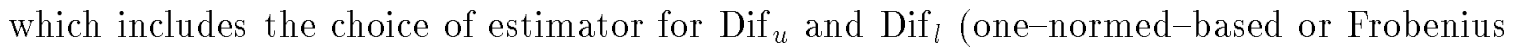
normed-based).

_TGSNA estimates the reciprocal condition numbers for specified eigenvalues and eigenvectors of a matrix pair $(A, B)$ in generalized Schur form. The logical array SELECT specifies the $M$ eigenpairs (all or a subset) for which condition numbers are required. The reciprocal values of estimates for the eigenvalue condition numbers $S(\lambda)$ (4.32) are reported in the array $\mathrm{S}$ and the corresponding reciprocal values of (Frobenius normed-based) estimates for the eigenvector condition numbers Dif $_{l}$ are reported in the array DIF. The calling sequence and the leading comment lines of DTGSNA are listed in Appendix D.

_GSRBB computes an algorithm-independent residual-based error bound for a pair (left and right) deflating subspaces of a matrix pair $(C, D)=Q^{H}(A, B) Z$, where $Q$ and $Z$ transform the original matrix pair $(A, B)$ to generalized Schur canonical form such that the M-by-M (1,1)-block of $(C, D)$ holds a selected cluster of eigenvalues. Optionally, a (Frobenius normed-based) estimate of $\operatorname{Dif}_{l}\left[\left(C_{11}, C_{11}\right),\left(D_{22}, D_{22}\right)\right]$ is reported in DIF. An estimate of the bound(s) (4.29), (4.30) is reported in RBB, and an estimate on $\eta$, which should be less than 1, is reported in CNDTN. RRES is the norm of the backward perturbation $\left\|H_{\text {opt }}\right\|(4.27)$ associated with the computed pair of deflating subspaces (i.e. the first $M$ columns of $Q$ and $Z$ ). INFO is set to 1 if CNDTN $\geq 1$. The calling sequence and the leading comment lines of DGSRBB are listed in Appendix E.

Notice, that it is only in _TGSEN where the user has the option to choose between onenormed-based or Frobenius normed-based estimates of $\operatorname{Dif}_{u}$ and Dif ${ }_{l}$. The estimation of Dif $_{l}$ for eigenvectors in _TGSNA and for deflating subspaces in _GSRBB make use of the less expensive but equally reliable Frobenius normed-based estimator (see Section 4.4).

\section{Computational Experiments}

We have performed an extensive testing of our software on problems ranging from wellconditioned to extremely ill-conditioned. In the following we report detailed results from a selection of test problems as well as a summary of results from the test programs. All results presented in the coming sections are computed on a Sun SPARC station 2 in double precision real (and complex) arithmetic with unit roundoff $\eta=\mathrm{EPS} \approx 2.2 \mathrm{D}-16$.

\subsection{Accuracy and Reliability Results}

We have chosen to illustrate the stability and accuracy of our software for a selection of problems "tagged" from 1 to 23 , where the basic operation is a swapping of two $2 \times 2$ blocks 
in a $4 \times 4$ matrix pair $(A, B)$ in generalized real Schur form

$$
A=\left[\begin{array}{cc}
A_{11} & A_{12} \\
0 & A_{22}
\end{array}\right], \quad B=\left[\begin{array}{cc}
B_{11} & B_{12} \\
0 & B_{22}
\end{array}\right] .
$$

The stability tests guarantees that the swapping of two diagonal blocks at most results in $O\left(\eta\|(A, B)\|_{F}\right)$ changes in the original matrix pair. In certain (ill-conditioned) cases this perturbation is enough to change individual eigenvalues a lot (e.g., a real multiple eigenvalue $\lambda$ of multiplicity $k$ might spread around in a circle in the complex plane with center $\lambda$ and radius $O\left(\eta^{1 / k}\right)$ ). However, for well-conditioned or only moderately ill-conditioned cases the change of the eigenvalues is an adequate measure on the reliability and accuracy of a reordering method. Besides, comparing different reordering methods (including the variants discussed in Section 3.2 and the $Q Z$-based method in [30]), we also report estimates of condition numbers and error bounds for eigenvalues and eigenspaces.

\subsubsection{Test Problems}

The first group of problems (1,6 and 11) are adopted from [2], and here we treat a standard eigenvalue problem as a generalized one, making it more ill-conditioned. We choose $A$ in $(A, B)$ as

$$
\left(\begin{array}{cccc}
2 & -87 & -20000 & 1000 \\
5 & 2 & -20000 & -1000 \\
& & 1 & -11 \\
& & 37 & 1
\end{array}\right),\left(\begin{array}{cccc}
1 & -3 & 3576 & 4888 \\
1 & 1 & -88 & -1440 \\
& & 1.0001 & -3 \\
& & 1.0001 & 1.0001
\end{array}\right),\left(\begin{array}{cccc}
1 & -100 & 400 & -1000 \\
0.001 & 1 & 1200 & -10 \\
& & 1.0001 & -3 \\
& & 100 & 1.0001
\end{array}\right),
$$

for problems 1, 6 and 11, respectively, and $B=I_{4}$. Notice that all $(1,2)$-blocks of $A$ have quite large norm.

The following matrix pair $(A, B)$ defines the second group of problems (2, 7 and 12):

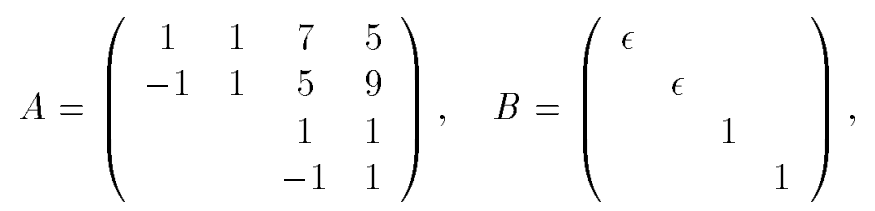

with the eigenvalues $\lambda_{1,2}=\epsilon^{-1} \pm i \epsilon^{-1}$ and $\lambda_{3,4}=1 \pm i$, which move along the lines starting at origin and passing $(1, i)$ and $(1,-i)$, respectively. When $0<\epsilon<1$ decreases, the eigenvalues $\lambda_{1,2}$ move away from $\lambda_{3,4}$ along these lines. Notice that $\operatorname{Dif}_{l}=\operatorname{Dif}_{u}$ is constant $\approx 0.7 E-2$ for problems 2, 7 and 12 corresponding to $\epsilon=1 \mathrm{E}-3,1 \mathrm{E}-9$ and $1 \mathrm{E}-15$, respectively.

The following matrix pair $(A, B)$ defines the third to fifth group of problems $((3,8,13)$, $(4,9,14)$ and $(5,10,15))$ :

$$
A=\left(\begin{array}{cccc}
1 & \delta & x & x \\
-\delta & 1 & 0 & x \\
& & 1+\epsilon & \delta \\
& & -\delta & 1+\epsilon
\end{array}\right), \quad B=\left(\begin{array}{cccc}
1 & 0 & x & x \\
0 & 1 & 0 & x \\
& & 1 & 0 \\
& & 0 & 1
\end{array}\right)
$$


Table 6.1: Problem characteristics, chordal distances and reciprocal condition numbers

\begin{tabular}{|cccc|cc|cc|ccc|}
\hline Tag & $\epsilon$ & $\delta$ & $x$ & $\mathcal{X}\left(\lambda_{1}, \lambda_{2}\right)$ & $\mathcal{X}\left(\lambda_{1}, \lambda_{3}\right)$ & $S\left(\lambda_{1,2}\right)$ & $S\left(\lambda_{3,4}\right)$ & $p^{-1}$ & $q^{-1}$ & $\mathrm{Dif}_{l}$ \\
\hline 1 & - & - & - & $1 \mathrm{E}-1$ & $3 \mathrm{E}-3$ & $6 \mathrm{E}-4$ & $6 \mathrm{E}-4$ & $2 \mathrm{E}-05$ & $2 \mathrm{E}-05$ & $1 \mathrm{E}-02$ \\
6 & - & - & - & $7 \mathrm{E}-1$ & $3 \mathrm{E}-4$ & $1 \mathrm{E}-6$ & $1 \mathrm{E}-6$ & $4 \mathrm{E}-07$ & $4 \mathrm{E}-07$ & $3 \mathrm{E}-04$ \\
11 & - & - & - & $7 \mathrm{E}-1$ & $3 \mathrm{E}-4$ & $3 \mathrm{E}-8$ & $3 \mathrm{E}-8$ & $2 \mathrm{E}-08$ & $2 \mathrm{E}-08$ & $1 \mathrm{E}-07$ \\
\hline 2 & $1 \mathrm{E}-3$ & - & - & $1 \mathrm{E}-3$ & $6 \mathrm{E}-1$ & $1 \mathrm{E}+0$ & $3 \mathrm{E}-1$ & $1 \mathrm{E}-01$ & $1 \mathrm{E}+00$ & $7 \mathrm{E}-01$ \\
7 & $1 \mathrm{E}-9$ & - & - & $1 \mathrm{E}-9$ & $6 \mathrm{E}-1$ & $1 \mathrm{E}+0$ & $3 \mathrm{E}-1$ & $1 \mathrm{E}-01$ & $1 \mathrm{E}+00$ & $7 \mathrm{E}-01$ \\
12 & $1 \mathrm{E}-15$ & - & - & $1 \mathrm{E}-15$ & $6 \mathrm{E}-1$ & $1 \mathrm{E}+0$ & $3 \mathrm{E}-1$ & $1 \mathrm{E}-01$ & $1 \mathrm{E}+00$ & $7 \mathrm{E}-01$ \\
\hline 3 & $1 \mathrm{E}-3$ & $1 \mathrm{E}+0$ & $5 \mathrm{E}-1$ & $7 \mathrm{E}-1$ & $3 \mathrm{E}-4$ & $4 \mathrm{E}-3$ & $4 \mathrm{E}-3$ & $2 \mathrm{E}-03$ & $2 \mathrm{E}-03$ & $4 \mathrm{E}-04$ \\
8 & $1 \mathrm{E}-3$ & $1 \mathrm{E}+3$ & $5 \mathrm{E}-1$ & $2 \mathrm{E}-3$ & $1 \mathrm{E}-9$ & $5 \mathrm{E}-3$ & $5 \mathrm{E}-3$ & $4 \mathrm{E}-06$ & $4 \mathrm{E}-06$ & $7 \mathrm{E}-07$ \\
13 & $1 \mathrm{E}-3$ & $1 \mathrm{E}+9$ & $5 \mathrm{E}-1$ & $2 \mathrm{E}-9$ & $1 \mathrm{E}-21$ & $5 \mathrm{E}-3$ & $5 \mathrm{E}-3$ & $8 \mathrm{E}-07$ & $8 \mathrm{E}-07$ & $8 \mathrm{E}-09$ \\
\hline 4 & $1 \mathrm{E}-1$ & $1 \mathrm{E}-1$ & $5 \mathrm{E}-1$ & $1 \mathrm{E}-1$ & $5 \mathrm{E}-2$ & $3 \mathrm{E}-1$ & $3 \mathrm{E}-1$ & $2 \mathrm{E}-01$ & $2 \mathrm{E}-01$ & $5 \mathrm{E}-02$ \\
9 & $1 \mathrm{E}-4$ & $1 \mathrm{E}-4$ & $5 \mathrm{E}-1$ & $1 \mathrm{E}-4$ & $5 \mathrm{E}-5$ & $3 \mathrm{E}-4$ & $3 \mathrm{E}-4$ & $2 \mathrm{E}-04$ & $2 \mathrm{E}-04$ & $5 \mathrm{E}-05$ \\
14 & $1 \mathrm{E}-9$ & $1 \mathrm{E}-9$ & $5 \mathrm{E}-1$ & $1 \mathrm{E}-9$ & $5 \mathrm{E}-10$ & $3 \mathrm{E}-9$ & $3 \mathrm{E}-9$ & $2 \mathrm{E}-09$ & $2 \mathrm{E}-09$ & $5 \mathrm{E}-10$ \\
\hline 5 & $1 \mathrm{E}-5$ & $1 \mathrm{E}-5$ & 0 & $1 \mathrm{E}-5$ & $5 \mathrm{E}-6$ & $1 \mathrm{E}+0$ & $1 \mathrm{E}+0$ & $1 \mathrm{E}+00$ & $1 \mathrm{E}+00$ & $5 \mathrm{E}-06$ \\
10 & $1 \mathrm{E}-5$ & $1 \mathrm{E}-5$ & $1 \mathrm{E}+2$ & $1 \mathrm{E}-5$ & $5 \mathrm{E}-6$ & $2 \mathrm{E}-7$ & $2 \mathrm{E}-7$ & $8 \mathrm{E}-08$ & $8 \mathrm{E}-08$ & $5 \mathrm{E}-06$ \\
15 & $1 \mathrm{E}-5$ & $1 \mathrm{E}-5$ & $1 \mathrm{E}+4$ & $1 \mathrm{E}-5$ & $5 \mathrm{E}-6$ & $2 \mathrm{E}-9$ & $2 \mathrm{E}-9$ & $8 \mathrm{E}-10$ & $8 \mathrm{E}-10$ & $5 \mathrm{E}-06$ \\
\hline 16 & $1 \mathrm{E}-3$ & - & - & 0 & $1 \mathrm{E}-3$ & $3 \mathrm{E}-22$ & $1 \mathrm{E}-9$ & $5 \mathrm{E}-10$ & $5 \mathrm{E}-10$ & $4 \mathrm{E}-10$ \\
17 & $1 \mathrm{E}-6$ & - & - & 0 & $1 \mathrm{E}-6$ & $2 \mathrm{E}-29$ & $1 \mathrm{E}-18$ & $5 \mathrm{E}-19$ & $5 \mathrm{E}-19$ & $2 \mathrm{E}-16$ \\
18 & $1 \mathrm{E}-3$ & - & - & $2 \mathrm{E}-7$ & $1 \mathrm{E}-3$ & $1 \mathrm{E}-13$ & $1 \mathrm{E}-9$ & $5 \mathrm{E}-10$ & $5 \mathrm{E}-10$ & $4 \mathrm{E}-10$ \\
19 & $1 \mathrm{E}-6$ & - & - & $1 \mathrm{E}-7$ & $1 \mathrm{E}-6$ & $8 \mathrm{E}-20$ & $1 \mathrm{E}-18$ & $9 \mathrm{E}-16$ & $9 \mathrm{E}-16$ & $5 \mathrm{E}-18$ \\
\hline 20 & $1 \mathrm{E}-3$ & - & - & 0 & $1 \mathrm{E}-3$ & $3 \mathrm{E}-16$ & $3 \mathrm{E}-16$ & $2 \mathrm{E}-06$ & $2 \mathrm{E}-06$ & $4 \mathrm{E}-10$ \\
21 & $1 \mathrm{E}-6$ & - & - & 0 & $1 \mathrm{E}-6$ & $3 \mathrm{E}-16$ & $3 \mathrm{E}-12$ & $2 \mathrm{E}-12$ & $2 \mathrm{E}-12$ & $2 \mathrm{E}-16$ \\
22 & $1 \mathrm{E}-3$ & - & - & $1 \mathrm{E}-7$ & $1 \mathrm{E}-3$ & $7 \mathrm{E}-8$ & $3 \mathrm{E}-6$ & $2 \mathrm{E}-06$ & $2 \mathrm{E}-06$ & $4 \mathrm{E}-10$ \\
23 & $1 \mathrm{E}-6$ & - & - & $2 \mathrm{E}-7$ & $1 \mathrm{E}-6$ & $5 \mathrm{E}-16$ & $3 \mathrm{E}-12$ & $7 \mathrm{E}-12$ & $7 \mathrm{E}-12$ & $2 \mathrm{E}-16$ \\
\hline
\end{tabular}


where $x$ represents a uniformly distributed random number $\in(-x, x)$. The eigenvalues of $(A, B)$ are $\lambda_{1,2}=1 \pm i \delta$ and $\lambda_{3,4}=1+\epsilon \pm i \delta$. For problems 3,8 and 13 we keep $\epsilon$ $(=1 \mathrm{E}-3)$ and $x(=0.5)$ constant and vary $\delta(=1,1 \mathrm{E}+3$ and $1 \mathrm{E}+9$, respectively). This implies that the eigenvalues move along the vertical lines through 1 and $1+\epsilon$ and form two separated clusters. For problems 4,9 and 14 we keep $x(=0.5)$ constant and varies $\epsilon=\delta(=1 \mathrm{E}-1,1 \mathrm{E}-4$ and $1 \mathrm{E}-9$, respectively $)$. For $\epsilon$ small enough we just have one cluster of close eigenvalues. For problems 5, 10 and 15 we keep $\epsilon=\delta(=1 \mathrm{E}-5)$ constant and vary $x(=0,1 \mathrm{E}+2$ and $1 \mathrm{E}+4$, respectively $)$. Problem 5 corresponds to a homogeneous generalized Sylvester equation and in this group of problems we only increase the departure from "block-diagonality", while keeping one cluster of close eigenvalues.

Problems $16-23$ are all modifications of a problem in [16], where $(A, B)$ is defined as [18]:

$$
A_{11}=\left(\begin{array}{ll}
1 & -1 \\
1 & -1
\end{array}\right), \quad A_{22}=A_{11}-\epsilon\left(\begin{array}{cc}
1+\epsilon & 0 \\
0 & -1
\end{array}\right), \quad B_{11}=B_{22}=I_{2} .
$$

For problems $16-19, \operatorname{col}\left(A_{12}\right)$ is equal to the left singular vector corresponding to $\sigma_{\min }\left(I_{2} \otimes\right.$ $\left.A_{11}-A_{22}^{T} \otimes I_{2}\right)$ and $B_{12}=I_{2}$. For problems $20-23, \operatorname{col}\left(A_{12}, B_{12}\right)$ is equal to the left singular vector corresponding to $\sigma_{\min }\left(Z_{u}\right)$, where $Z_{u}$ is defined in (4.13). The parameter $\epsilon=1 \mathrm{E}-3$ for problems 16, 18, 20 and 22 and $\epsilon=1 \mathrm{E}-6$ for problems 17, 19, 21 and 23 . The last two problems in each group (i.e. 18,19 and 22,23 ) are orthogonally equivalent to the first two (i.e. 16,17 and 20,21 , respectively).

Table 6.1 shows problem characteristics, including the chordal distance between $\lambda_{1}$ and $\lambda_{2}$ and $\lambda_{1}$ and $\lambda_{3}$, reciprocal values of the individual condition numbers for the two complex conjugate pairs (computed by DTGSNA), reciprocal values of (left and right) projector norms (computed by DTGSEN) and exact values of $\operatorname{Dif}_{l}$ (computed as $\sigma_{\min }\left(Z_{l}\right)$ ). Several of these problems represent ill-conditioned eigenvalue problems, where both individual eigenvalues, the cluster of eigenvalues in the $(1,1)$-block and associated pair of deflating subspaces have large condition numbers.

\subsubsection{Comparing Different Reordering Methods}

We report results from the three method variants discussed in Section 3.2 and the $Q Z-$ based Algorithm 590 [30]. Results from a prototype implementation in Matlab of Method 1 in Section 3.2 (the generic method) has been reported earlier [18]. Besides, the first and the last two groups of problems above, results were reported for problems with finite and infinite eigenvalues (simple as well as defective).

Let $(\bar{A}, \bar{B})$ denote the computed matrix pair after the swapping of two diagonal blocks and $\bar{Q}, \bar{Z}$ be the computed transformation matrices that perform the requested reordering. Now, we consider the following questions:

- How close is $\left(\bar{Q} \bar{A} \bar{Z}^{T}, \bar{Q} \bar{B} \bar{Z}^{T}\right)$ to the original matrix pair $(A, B)$ ?

- How nearly orthogonal are the computed transformation matrices $\bar{Q}$ and $\bar{Z}$ ?

- How close are the eigenvalues of $\left(A_{i i}, B_{i i}\right)$ (before the swapping) and $\left(\bar{A}_{i i}, \bar{B}_{i i}\right)$ (after the swapping)? 
To answer the first two questions we measure the quantities

$$
\begin{gathered}
E_{A, B}=\frac{\left\|\left(A-\bar{Q} \bar{A} \bar{Z}^{T}, B-\bar{Q} \bar{B} \bar{Z}^{T}\right)\right\|_{F}}{\eta\|(A, B)\|_{F}}, \\
\left\|\left(\bar{A}_{21}, \bar{B}_{21}\right)\right\|_{F}, \\
E_{Q}=\frac{\left\|I_{n}-\bar{Q}^{T} \bar{Q}\right\|_{F}}{\eta}, \quad E_{Z}=\frac{\left\|I_{n}-\bar{Z}^{T} \bar{Z}\right\|_{F}}{\eta},
\end{gathered}
$$

where $\eta$ is the relative machine precision. Ideally, $E_{A, B}$ and $E_{Q}, E_{Z}$ should be of size $O(1)$ and the norms of the $(2,1)$-blocks $(6.3)$ should be of size $O(\eta)$.

In tables 7.1, 7.2, 7.4 and 7.5 we display these quantities and the absolute backward error $\|(E, F)\|_{F} \equiv\left\|\left(A-\bar{Q} \bar{A} \bar{Z}^{T}, B-\bar{Q} \bar{B} \bar{Z}^{T}\right)\right\|_{F}$ for Method 1, Method 2, Method 3 and Algorithm 590 , respectively. In summary, Method 3 and Algorithm 590 (which both are based on $Q Z$ iterations) perform the swapping in all cases with small backward errors in $(A, B)$. The direct methods 1 and 2 reject the swaps for problems 18, 19, 22 and 23. Moreover, Method 2 rejects the swap for problem 13 . In Table 7.3 we show computed stability test values and tolerances. We accept a swap if and only if both the weak and the strong stability test values are smaller than toll $=$ tol2 (see Section 3.2). If a swap is rejected due to (severe) ill-conditioning we can still impose a swap by increasing the tolerances in the stability tests, which is done for methods 1 and 2 to get the results for the "rejected swaps" in tables 7.1 and 7.2 .

To answer the last question we display computed eigenvalues after the reordering. Numbers in bold font show the absolute error in computed eigenvalues. Table 6.2 and Table 6.3 illustrate the well-known fact that small backward errors do not necessarily imply small errors in the computed eigenvalues. Here the direct Method 2 produces much more accurate eigenvalues than Algorithm 590. For these examples Method 1 and Method 2 produce

\begin{tabular}{|c|c|c|}
\hline Tag & Alg. 590 - Real parts & Alg. 590 - Imaginary parts \\
\hline \multirow[t]{2}{*}{1} & $0.1000000000637105 \mathrm{E}+01$ & $\pm 0.2017424100339241 \mathrm{E}+02$ \\
\hline & $0.1999999999360595 \mathrm{E}+01$ & $\pm 0.2085665361375716 \mathrm{E}+02$ \\
\hline \multirow[t]{2}{*}{6} & $0.1000999999900501 \mathrm{E}+01$ & $\pm 0.1732916616366925 \mathrm{E}+01$ \\
\hline & $0.10000000000 \mathbf{9 9 3 2 9 E}+01$ & $\pm 0.1732050807522568 \mathrm{E}+01$ \\
\hline \multirow[t]{2}{*}{11} & $0.1000998004480817 \mathrm{E}+01$ & $\pm 0.1000004238589241 \mathrm{E}+01$ \\
\hline & $0.1000001995519146 \mathrm{E}+01$ & $\pm 0.9999957594733729 \mathrm{E}+00$ \\
\hline Tag & Method $2-$ Real parts & Method 2 - Imaginary parts \\
\hline \multirow[t]{2}{*}{1} & $0.1000000000000001 \mathrm{E}+01$ & $\pm 0.2017424100183201 \mathrm{E}+02$ \\
\hline & $0.2000000000000000 \mathrm{E}+01$ & $\pm 0.2085665361461420 \mathrm{E}+02$ \\
\hline \multirow[t]{2}{*}{6} & $0.1001000000000000 \mathrm{E}+01$ & $\pm 0.1732916616574496 \mathrm{E}+01$ \\
\hline & $0.9999999999999999 \mathrm{E}+00$ & $\pm 0.1732050807568877 \mathrm{E}+01$ \\
\hline \multirow[t]{2}{*}{11} & $0.1000999999999995 \mathrm{E}+01$ & $\pm 0.1000000000000 \mathbf{1 8 6} \mathrm{E}+01$ \\
\hline & $0.9999999999999991 \mathrm{E}+00$ & $\pm 0.9999999999999453 \mathrm{E}+00$ \\
\hline
\end{tabular}
exactly the same eigenvalues but this is not always the case as we will see later.

Table 6.2: Eigenvalues after reordering for problems 1,6 and 11 
Table 6.3: Eigenvalues after reordering for problems 5, 10 and 15

\begin{tabular}{|r|cc|}
\hline Tag & Alg. $590-$ Real parts & Alg. 590 - Imaginary parts \\
\hline 5 & $0.1000010000000000 \mathrm{E}+01$ & $\pm 0.1000000000006551 \mathrm{E}-04$ \\
& $0.1000000000000001 \mathrm{E}+01$ & $\pm 0.9999999999996121 \mathrm{E}-05$ \\
\hline 10 & $0.1000010015362113 \mathrm{E}+01$ & $\pm 0.9986863986519929 \mathrm{E}-05$ \\
& $0.9999999846379060 \mathrm{E}+00$ & $\pm 0.1002579812190853 \mathrm{E}-04$ \\
\hline 15 & $0.1000063691043548 \mathrm{E}+01$ & $\pm \mathbf{0 . 0 0 0 0 0 0 0 0 0 0 0 0 0 0 0 0} \mathrm{E}+00$ \\
& $0.1000011414827676 \mathrm{E}+01$ & $\pm 0.1394322538135408 \mathrm{E}-04$ \\
\hline Tag & Method $2-$ Real parts & Method $2-$ Imaginary parts \\
\hline 5 & $0.1000010000000000 \mathrm{E}+01$ & $\pm 0.1000000000000000 \mathrm{E}-04$ \\
& $0.1000000000000000 \mathrm{E}+01$ & $\pm 0.1000000000000000 \mathrm{E}-04$ \\
\hline 10 & $0.1000010000000000 \mathrm{E}+01$ & $\pm 0.9999999999295905 \mathrm{E}-05$ \\
& $0.9999999999999998 \mathrm{E}+00$ & $\pm 0.100000000000 \mathbf{3 9 1 3} \mathrm{E}-04$ \\
\hline 15 & $0.1000010000000000 \mathrm{E}+01$ & $\pm 0.9999999999775261 \mathrm{E}-05$ \\
& $0.1000000000000001 \mathrm{E}+01$ & $\pm 0.10000000000 \mathbf{9 4 1 3 1} \mathrm{E}-04$ \\
\hline
\end{tabular}

Table 6.4: Eigenvalues after reordering for problem 12

\begin{tabular}{|c|cc|}
\hline Method & Real part & Imaginary part \\
\hline 590 & $0.9999999999999998 \mathrm{E}+00$ & $\pm 0.9999999999999994 \mathrm{E}+00$ \\
& $0.100000 \mathbf{5 0 7 4 6 0 3 3 7 2} \mathrm{E}+11$ & $\pm 0.99999999996 \mathbf{6 2 1 3 4} \mathrm{E}+10$ \\
\hline 3 & $0.9999999999998981 \mathrm{E}+00$ & $\pm 0.999999999999997 \mathbf{E}+00$ \\
& $0.1000000000000006 \mathrm{E}+11$ & $\pm 0.9999999999999947 \mathrm{E}+10$ \\
\hline 2 & $0.9999999999998981 \mathrm{E}+00$ & $\pm 0.9999999999999993 \mathrm{E}+00$ \\
$B=Q R$ & $0.1000000000000003 \mathrm{E}+11$ & $\pm 0.9999999999999968 \mathrm{E}+10$ \\
\hline 1 & $0.9999999999998976 \mathrm{E}+00$ & $\pm 0.9999999999999988 \mathrm{E}+00$ \\
& $0.99999 \mathbf{1 7 7 8 8 4 5 4 4 9 8} \mathrm{E}+10$ & $\pm 0.1000004 \mathbf{6 9 3 4 8 0 3 0} \mathrm{E}+11$ \\
\hline
\end{tabular}


In Table 6.4 we show that Method 2 and Method 3 compute the most accurate eigenvalues for problem 12, and are superior to Method 1 and Algorithm 590. Moreover, we have not found any example where Method 1 preserves eigenvalues better than Method 2.

Notice that in all cases where Method 2 rejected a swap (except problem 13), Algorithm 590 produced eigenvalues with no accuracy at all (as all methods did when a rejected swap was imposed!). For problem 13, Algorithm 590 produced eigenvalues to half machine precision after the reordering. On the other hand, if SMIN, the threshold for checking nonzero diagonal entries in the $L U$ factorization routine DGELUF in the generalized Sylvester solver (see [19]) is set to the relative machine precision $\eta$ instead of $\eta\|Z\|_{M}$ (where $\|Z\|_{M}$ is the modulus of the largest element in the matrix to factorize), Methods 1-3 perform the swap of the eigenvalues to almost full machine precision. These results are "tagged" $13 *$ in tables $7.1,7.2,7.3$ and 7.6 .

\subsubsection{Results from Condition Estimation and Error Bounds}

In Table 6.1 we reported some condition estimation results, namely reciprocal values of the individual condition numbers for the two complex conjugate pairs (computed by DTGSNA) and reciprocal values of (left and right) projector norms (computed by DTGSEN). The exact values of $\operatorname{Dif}_{l}=\operatorname{Dif}_{u}$ (computed as $\sigma_{\min }\left(Z_{l}\right)$ ) were also displayed. In Table 7.6 we display $\operatorname{Dif}_{u}$ before and after the reordering for our selection of problems. Moreover, we show the ratios $\operatorname{Dif}_{u} /$ DIF (1)-F and $\operatorname{Dif}_{u} /$ DIF (1)-1, where DIF (1)-F and DIF(1)-1 are the Frobenius normed-based and one-normed-based estimates of Dif $f_{u}$, respectively. We see that 20 examples are within a factor 10 and the remaining 3 examples are within a factor 100 of the exact value of $\operatorname{Dif}_{u}$. In the last two columns we report estimates of the reciprocal values of Dif ${ }_{l}$, the condition number for the individual eigenvectors corresponding to the complex conjugate pair $\lambda_{1,2}$ computed by DTGSNA and ZTGSNA, respectively.

For a more complete comparison between our Dif $x_{x}$ estimators $(x=l, u)$ including accuracy, performance and reliability results we refer to [19]. Moreover, estimates of condition numbers and error bounds are also checked by the test programs discussed in the next section.

\subsection{A Summary of the Results from the Test Programs}

We have developed two test programs_CHK3 and _CHK4 for testing and verification of _TGSEN and_GSRBB, and _TGSNA, respectively.

The test program _CHK3 verifies that the backward error is small, the transformation matrices $\bar{Q}$ and $\bar{Z}$ that performed the reordering are orthogonal (unitary), the estimated values DIF (1:2) do not differ too much from the true values of $\operatorname{Dif}_{u}$ and $\operatorname{Dif}_{l}$, respectively, the chordal distance between "the same" eigenvalues before and after the reordering is small, and that the norm of the $(2,1)$-blocks of the reordered pencil is small. The scheme is to initialize $A_{11}, A_{22}, B_{11}, B_{22}, R$ and $L$ and they define the (1,2)-blocks $A_{12}$ and $B_{12}$ (as in (4.12)). The program reorders all eigenvalues in $\left(A_{22}, B_{22}\right)$ to the $(1,1)$-block of the matrix pair and checks if everything went well.

The test program _CHK4 verifies that the computed eigenvalue and eigenvector error bounds hold. This is accomplished by using pencils for which the exact eigenvalues and eigenvectors are known. 


\subsubsection{Test problems for_CHK3}

The test problems for _CHK3 are chosen from five different types, generated as follows. The size $n$ of a problem is chosen $\leq 10$ and $m=1,2, \ldots, 9 ; n-m=1, \ldots, 10-m$ ), where $\left(A_{11}, B_{11}\right)$ are $m \times m$ and $\left(A_{22}, B_{22}\right)$ are $(n-m) \times(n-m)$. Note that Type 1 corresponds to a standard eigenvalue problem but the others are regular generalized eigenvalue problems.

Type 1: Upper triangular problem adapted from [31]: $A_{11}=J_{m}(1,-1), B_{11}=I_{m}$ and $A_{22}=J_{n-m}(1-\alpha, 1), B_{22}=I_{n-m}$, where $J_{k}(d, s)$ denotes a Jordan block of dimension $k$ with $d$ and $s$ as diagonal and superdiagonal elements, respectively. In our tests we use $\alpha=\sqrt{\mathrm{EPS}}$, and $1 / \sqrt{\mathrm{EPS}}$. For $0<\alpha<1$, the size of Dif is $O\left(\alpha^{n-1}\right)$. The entries in $L=R$ are chosen as $r_{i j}=20(0.5-\sin (i / j))$, where $i=1, \ldots, m$ and $j=1, \ldots, n$.

Type 2: Upper triangular problem with

$$
\begin{gathered}
a_{i j}=2(0.5-\sin (i)), \quad b_{i j}=2(0.5-\sin (i \cdot j)), \quad i=1, \ldots, m, \quad j=i, \ldots, m . \\
a_{i j}=2(0.5-\sin (i+j)), \quad b_{i j}=2(0.5-\sin (j)), \quad i=m+1, \ldots, n-m, \quad j=i, \ldots, n-m . \\
r_{i j}=20(0.5-\sin (i \cdot j)), \quad l_{i j}=20(0.5-\sin (i+j)), \quad i=1, \ldots, m, \quad j=1, \ldots, n-m .
\end{gathered}
$$

Type 3: Quasi upper triangular problem, where the entries in $A, B, R$ and $L$ at first are chosen as for Type 2. Then each second diagonal block in $A_{11}$ and each third diagonal block in $A_{22}$ are made $2 \times 2$ by setting $a_{k+1, k+1}=a_{k k}$ and $a_{k+1, k}=-\sin \left(a_{k, k+1}\right)$ for appropriate values of $k$.

Type 4: Dense problem at block level, with

$$
\begin{gathered}
a_{i j}=20(0.5-\sin (i \cdot j)), \quad b_{i j}=2(0.5-\sin (i+j)), \quad i=1, \ldots, m, \quad j=1, \ldots, m . \\
a_{i j}=20(0.5-\sin (i+j)), \quad b_{i j}=2(0.5-\sin (i \cdot j)), \quad i=m+1, \ldots, n-m, \quad j=m+1, \ldots, n-m . \\
r_{i j}=2(0.5-\sin (i / j)), \quad l_{i j}=20(0.5-\sin (i \cdot j)), \quad i=1, \ldots, m, \quad j=1, \ldots, n-m .
\end{gathered}
$$

Type 5: $(A, B)$ has potentially close or common eigenvalues, and large or very large departure from block diagonality. First $A_{11}$ is chosen as the $m \times m$ leading submatrix of $A_{1}$, where

$$
A_{1}=\left(\begin{array}{ccccccccc}
1 & \beta & & & & & & & \\
-\beta & 1 & & & & & & & \\
& & 1+\delta & \beta & & & & & \\
& & -\beta & 1+\delta & & & & & \\
& & & & \delta & 1 & & & \\
& & & & -1 & \delta & & & \\
& & & & & & -\delta & 1 & \\
& & & & & & -1 & -\delta & \\
& & & & & & & & 1
\end{array}\right),
$$


then $A_{22}$ is chosen as the $(n-m) \times(n-m)$ leading submatrix of $A_{2}$, where

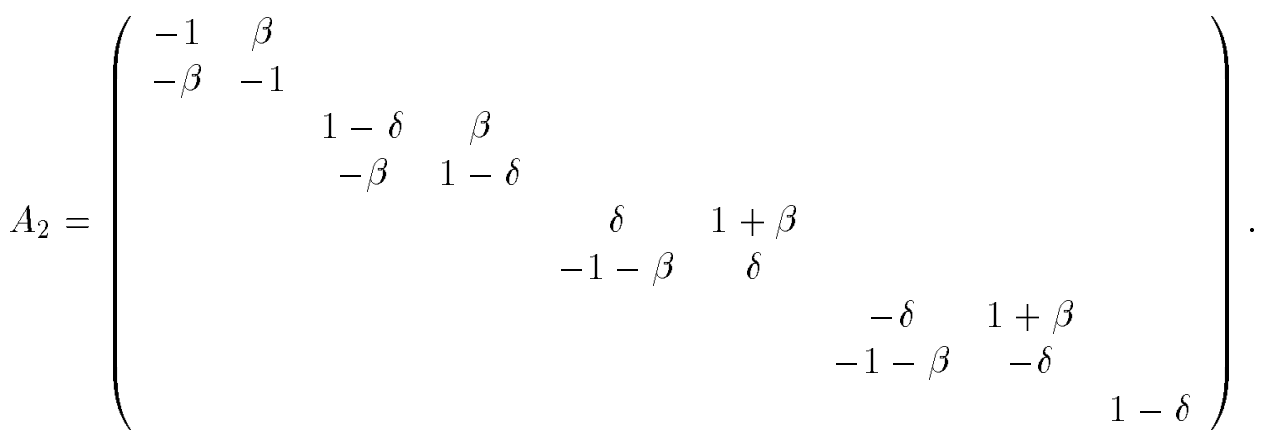

$B_{11}$ and $B_{22}$ are chosen as the identity matrices $I_{m}$ and $I_{n-m}$, respectively. The values of $\beta=20 / \alpha$ and $\delta=-1.5 / \alpha$ are used for $\alpha=\sqrt{\text { EPS }}$ and $1 / \sqrt{E P S}$. The entries in $R$ and $L$ are chosen as $r_{i j}=\alpha(0.5-\sin (i \cdot j)) / 20$ and $l_{i j}=\alpha(0.5-\sin (i+j)) / 20$, where $i=$ $1, \ldots, m$ and $j=1, \ldots, n-m$. All integer values $m, n-m \geq 0$ such that $2 \leq n \leq 10$ are chosen.

\subsubsection{A Summary of the Results from _CHK3}

The results from the real and complex test runs were similar, only that 60 of the 2700 problems had to be omitted in the complex case because the LAPACK routine CGEGS did not manage to compute the generalized Schur form for these examples. We run both programs on two different target machines, both supporting IEEE floating point standards. A Sun SPARC station only using non-optimized BLAS, and an IBM RS6000/530, where we used the ESSL library for BLAS calls. All programs were compiled with the -O option. The relative machine precision EPS is $\approx 2.22 D-16$ for both machines. In the following, we always start to list a number for Sun SPARC followed by the corresponding number (in parentheses) for IBM RS6000/530.

A total number of 2700 test configurations (including different functionality tests for some problems) are generated in DCHK3 and in total we do around 20790 adjacent swaps. The minimum and maximum values of the backward error were $0.458 \mathrm{D}-19$ (0.458D-19) and $0.193 \mathrm{D}-14$ (0.169D-14), respectively. Minimum and maximum values of $\left\|I_{n}-\bar{Q}{ }^{T} \bar{Q}\right\|_{F}$ were $0.0(0.0)$ and $0.375 \mathrm{D}-14(0.326 \mathrm{D}-14)$, respectively. Minimum and maximum values of $\left\|I_{n}-\bar{Z}^{T} \bar{Z}\right\|_{F}$ were $0.0(0.0)$ and $0.335 \mathrm{D}-14$ (0.289D-14), respectively. These residuals were larger than $10 \times$ EPS in $120(114)$ and $144(78)$ cases out of 2700 , respectively. The estimates of $\operatorname{Dif}_{u}$ and Dif $_{l}$ differed more than a factor 100 from true values in 170 (184) cases out of 1800 , respectively. The maximum value of $\operatorname{Dif}_{x}$ is 1.41 and the corresponding estimate DIF $(*)$ was 1.00 for both machines. The minimum value of Dif $x$ is $0.210 \mathrm{D}-19$ (0.480D-19) and corresponding estimate DIF $(*)$ was computed as $0.774 \mathrm{D}-19$ (0.774D-19).

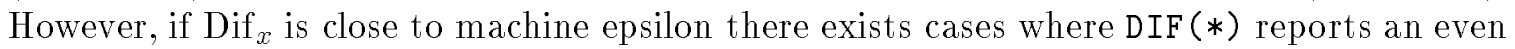
smaller value (but signals the ill-conditioning correctly). The minimum and maximum values of $\|\left(A_{21}, B_{21} \|_{F}\right.$ (i.e. RRES) were 0.0 and $0.360 \mathrm{D}-6$ for both machines and for the same problem. RRES was larger than $\operatorname{EPS}\|(A, B)\|_{F}$ in $18(0)$ cases out of 1350 , respectively. The chordal distance, $\mathcal{X}\left(\lambda_{\text {before }}, \lambda_{\text {after }}\right)$ was larger than $\operatorname{EPS}\|(A, B)\|_{F}$ in $192(192)$ cases out of 39600 but never larger than 0.226D-6 (0.266D-6), for the two machines. Minimum 
and maximum values of the residual based bound on the acute angle between exact and computed deflating subspaces, RBB, were $0.0(0.0)$ and $0.184 \mathrm{D}-6(0.184 \mathrm{D}-6)$, respectively. The maximum value occurred for the very last problem (i.e. Type 5 with $m=9, n-m=1$

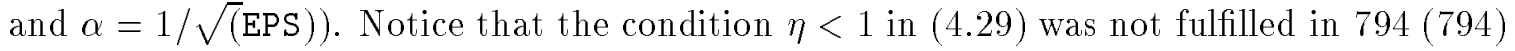
cases out of 1350 , showing that it is stronger than necessary (since all error bounds gave meaningful information).

We also substituted the sin-function in all problems and $\beta$ and $\delta$ in problems of Type 5 with a uniformly random number $\in(0,1)$, giving similar results as reported above.

\subsubsection{Test Problems and a Summary of the Results from _CHK4}

The program _CHK4 for testing _TGSNA checks how much the estimates $\mathbf{S}$ of the reciprocal value for the eigenvalue condition number $S(\lambda)$ (4.32) differ from the ones computed by using the exact (known) eigenvectors. The program also checks how much the estimates DIF of the reciprocal value for the eigenvector condition number Dif ${ }_{l}$ differ from the exact computed values $\sigma_{\min }\left(Z_{l}\right)$. In the tests two types of matrix pairs $(A, B) \equiv Y^{-H}\left(D_{a}, D_{b}\right) X^{-1}$ are used:

Type 1:

$$
D_{a}=\left(\begin{array}{ccccc}
1+\alpha & & & & \\
& 2+\alpha & & & \\
& & 3+\alpha & & \\
& & & 4+\alpha & \\
& & & & 5+\alpha
\end{array}\right)
$$

\section{Type 2:}

$$
D_{a}=\left(\begin{array}{ccccc}
1 & -1 & & \\
1 & 1 & & & \\
& & 1 & & \\
& & & 1+\alpha & 1+\beta \\
& & & -1-\beta & 1+\alpha
\end{array}\right)
$$

For both types $D_{b}=I_{5}$ and the exact left and right eigenvectors of $(A, B)$ are the rows and columns of

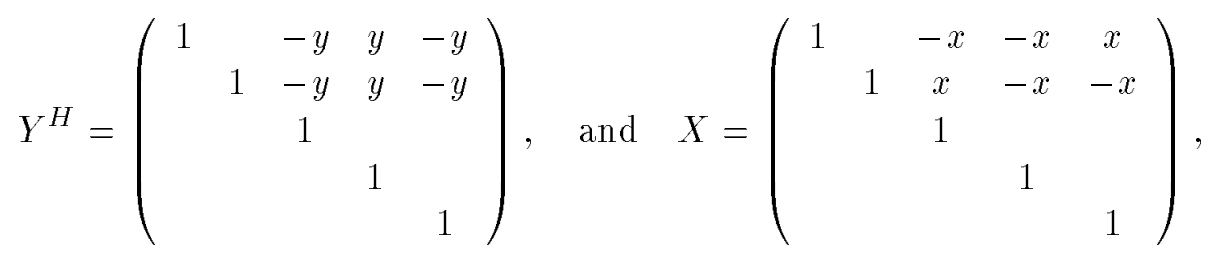

respectively, where $\alpha, \beta, x$ and $y$ are given all values independently of each other from $\left\{\mathrm{EPS}^{1 / 4}, 0.1,1,10, \mathrm{EPS}^{-1 / 4}\right\}$. So, a total of 1250 different pencils $(A, B)$ are generated in the tests. Note that $B \neq I_{5}$ in $(A, B)=Y^{-H}\left(D_{a}, D_{b}\right) X^{-1}$.

In summary, the test results are good. The ratio between $\mathbf{S}$ and the corresponding $S(\lambda)$ computed by using the exact (known) eigenvectors is 1.0 up to 8 decimal digits for all 
examples. The reciprocal values for the eigenvector condition numbers Dif $l$ differ less than a factor 100 from the exact computed value $\sigma_{\min }\left(Z_{l}\right)$ in about $90 \%, 94 \%, 99 \%$ and $100 \%$ of the 2500 cases for the four different data types D, S, Z and C, respectively. This verifies that

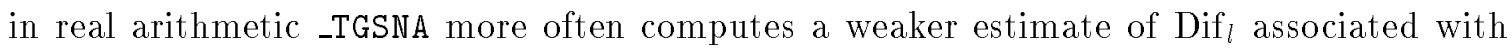
complex conjugate pairs of eigenvalues than in complex arithmetic (see Section 4.4.2).

\section{Some Conclusions}

Our error analysis of the direct methods and computational experiments (presented in Section 6) give us support to state the following conclusions about our algorithms and software.

- Accuracy and reliability results comparing different reordering methods (Method 1, Method 2 and Method 3 discussed in Section 3.2 and Algorithm 590 [30]) show that Method 2 is to prefer. It is a direct method which is very reliable and it also computes the most accurate eigenvalues of the four methods. Method 2 is implemented in the software presented in Section 5.

- The numerical stability is guaranteed and controlled by computing the size of the backward error and rejecting the swap if it exceeds a certain threshold.

As mentioned earlier, the generalized eigenvalue problem (as well as the standard unsymmetric problem) is potentially ill-conditioned in the sense that eigenvalues and eigenspaces may change drastically even under small perturbations of the data. If we insist on performing a reordering of $\left(S_{11}, T_{11}\right)$ and $\left(S_{22}, T_{22}\right)$ for an ill-conditioned problem, we may destroy any spectral information in $A-\lambda B$. Close eigenvalues or small separation between $\left(S_{11}, T_{11}\right)$ and $\left(S_{22}, T_{22}\right)$ are not enough for rejecting a swap. It is the sensitivity of the eigenspaces that matters most, which in turn is perfectly signaled by the norm of the solutions $L$ and $R$ to the associated generalized Sylvester equation for the direct methods discussed here.

- Qualitative results from our test software on both well-conditioned and ill-conditioned problems, including estimates of reciprocal values of condition numbers for individual eigenvalues, a cluster of eigenvalues, (left and right) eigenvectors, and a pair of (left and right) deflating subspaces, show the reliability and robustness of the algorithms and software presented.

\section{Acknowledgements}

We are grateful to Zhaojun Bai and Jim Demmel for fruitful discussions on this work. A special thank to Ji-guang Sun for constructive discussions on error bounds for the generalized eigenvalue problem and comments on an early version of the manuscript.

Financial support has been received from the Swedish National Board of Industrial and Technical Development under grant NUTEK 89-02578P. 


\section{References}

[1] E. Anderson, Z. Bai, C. Bischof, J. Demmel, J. Dongarra, J. Du Croz, A. Greenbaum, S. Hammarling, A. McKenney, S. Ostrouchov, and D. Sorensen. LAPACK Users' Guide. Society for Industrial and Applied Mathematics, Philadelphia, 1992.

[2] Z. Bai and J. Demmel. On swapping diagonal blocks in real Schur form. Lin. Alg. Appl., 186:73-95, 1993.

[3] Z. Bai, J. Demmel, and McKenney A. On computing condition numbers for the nonsymmetric eigenproblem. ACM Trans. Math. Software, 19(2):202-223, 1993.

[4] H. Bart, I. Gohberg, M. Kaashoek, and P. Van Dooren. Factorization of transfer functions. SIAM J. Control Optim., 18:675-696, June 1980.

[5] A. Bojanczyk, G. Golub, and P. Van Dooren. The periodic Schur decomposition. A1gorithms and applications. SCCM Inter. Rept. NA-92-07, Stanford University, August 1992.

[6] J. Demmel. The condition number of equivalence transformations that block diagonalize matrix pencils. SIAM J. Num. Anal., 20(3):599-610, June 1983.

[7] J. Demmel. On condition numbers and the distance to the nearest ill-posed problem. Num. Math., 51(3):251-289, July 1987.

[8] J. Demmel and B. Kăgström. Stable eigendecompositions of matrix pencils $A-\lambda B$. Report UMINF-118.84, Institute of Information Processing, University of Umeå, S-901 87 Umeå, Sweden, 1984.

[9] J. Demmel and B. Kågström. Computing stable eigendecompositions of matrix pencils. Lin. Alg. Appl., 88/89:139-186, April 1987.

[10] J. Demmel and B. Kågström. The Generalized Schur Decomposition of an Arbitrary Pencil $A-\lambda B$ : Robust Software with Error Bounds and Applications. Part I: Theory and Algorithms. ACM Trans. Math. Software, Vol.19(No. 2):160-174, June 1993.

[11] J. Demmel and B. Kågström. The Generalized Schur Decomposition of an Arbitrary Pencil $A-\lambda B$ : Robust Software with Error Bounds and Applications. Part II: Software and Applications. ACM Trans. Math. Software, Vol.19(No. 2):175-201, June 1993.

[12] F. Gantmacher. The Theory of Matrices, Vol. I and II (transl.). Chelsea, New York, 1959.

[13] G. Golub and C. Van Loan. Matrix Computations. Second Edition. Johns Hopkins University Press, Baltimore, MD, 1989.

[14] W. W. Hager. Condition estimators. SIAM J. Sci. Stat. Comput., 5:311-316, 1984.

[15] N. J. Higham. ALGORITHM 674: Fortran codes for estimating the one-norm of a real or complex matrix, with applications to condition estimation. ACM Trans. Math. Software, 15(2):168, 1989. 
[16] N. J. Higham. Perturbation theory and backward error analysis for $A X-X B=C$. BIT, 33(1):124-136, 1993.

[17] B. Kågström. A Perturbation Analysis of the Generalized Sylvester Equation. Report UMINF-92.17, Institute of Information Processing, University of Umeà, S-901 87 Umeå, Sweden, 1992. To appear in SIAM J. on Matrix Anal. Appl., Vol. 15, 1994.

[18] B. Kàgström. A Direct Method for Reordering Eigenvalues in the Generalized Real Schur Form of a Regular Matrix Pair $(A, B)$. In M.S. Moonen, G.H. Golub, and B.L.R. De Moor, editors, Linear Algebra for Large Scale and Real-Time Applications, pages 195-218. Kluwer Academic Publishers, Amsterdam, 1993.

[19] B. Kågström and P. Poromaa. LAPACK-Style Algorithms and Software for Solving the Generalized Sylvester Equation and Estimating the Separation between Regular Matrix Pairs. Report UMINF-93.23, Institute of Information Processing, University of Umeå, S-901 87 Umeå, Sweden, November, 1993. Also as LAPACK Working Note LAWN 75.

[20] B. Kågström and P. Van Dooren. A generalized state-space approach for the additive decomposition of a transfer matrix. Int. J. Numerical Linear Algebra with Applications, $1(2): 165-181,1992$.

[21] B. Kågström and L. Westin. Generalized Schur methods with condition estimators for solving the generalized Sylvester equation. IEEE Trans. Autom. Contr., 34(4):745-751, 1989.

[22] A. Laub. A Schur method for solving algebraic Riccati equations. IEEE Trans. Autom. Contr., AC-24:913-921, 1979.

[23] C. Moler and G. Stewart. An algorithm for the generalized matrix eigenvalue problem. SIAM J. Numer. Anal., 10:241-256, 1973.

[24] J. L. Rigal and J. Gaches. On the compatability of a given solution with the data of a linear system. J. Assoc. Comput. Mach., 14:543-548, 1967.

[25] G. W. Stewart. Error and perturbation bounds for subspaces associated with certain eigenvalue problems. SIAM Review, 15(4):727-764, Oct 1973.

[26] G. W. Stewart and J.-G. Sun. Matrix Perturbation Theory. Academic Press, New York, 1990.

[27] J.-G. Sun. Perturbation expansions for invariant subspaces. Lin. Alg. Appl., 153:85-97, 1991.

[28] J.-G. Sun. Backward perturbation analysis of certain characteristic subspaces. Num. Math., 65:357-382, 1993.

[29] P. Van Dooren. A generalized eigenvalue approach for solving Riccati equations. SIAM J. Sci. Stat. Comp., 2:121-135, 1981. 
[30] P. Van Dooren. ALGORITHM 590: DSUBSP and EXCHQZ: Fortran routines for computing deflating subspaces with specified spectrum. ACM Trans. Math. Software, 8:376-382, 1982. and (Corrections) Vol. 4, No. 4, p 787, 1983.

[31] J. Varah. On the separation of two matrices. SIAM J. Numer. Anal., 16:216-222, 1979.

[32] J. H. Wilkinson. Kronecker's canonical form and the QZ algorithm. Lin. Alg. Appl., $28: 285-303,1979$.

Table 7.1: Computed errors after the reordering using Method 1

\begin{tabular}{|c|ccccc|}
\hline Tag & $\left\|A_{21}, B_{21}\right\|_{F}$ & $E_{A, B}$ & $E_{Q}$ & $E_{Z}$ & $\|(E, F)\|_{F}$ \\
\hline 1 & $2 \mathrm{E}-18$ & $4 \mathrm{E}+00$ & $4 \mathrm{E}+00$ & $3 \mathrm{E}+00$ & $3 \mathrm{E}-11$ \\
6 & $1 \mathrm{E}-21$ & $3 \mathrm{E}+00$ & $3 \mathrm{E}+00$ & $5 \mathrm{E}+00$ & $4 \mathrm{E}-12$ \\
11 & $4 \mathrm{E}-19$ & $2 \mathrm{E}+00$ & $2 \mathrm{E}+00$ & $2 \mathrm{E}+00$ & $5 \mathrm{E}-13$ \\
\hline 2 & $4 \mathrm{E}-16$ & $2 \mathrm{E}+00$ & $8 \mathrm{E}+00$ & $3 \mathrm{E}+00$ & $7 \mathrm{E}-15$ \\
7 & $5 \mathrm{E}-16$ & $2 \mathrm{E}+00$ & $3 \mathrm{E}+00$ & $2 \mathrm{E}+00$ & $5 \mathrm{E}-15$ \\
12 & $2 \mathrm{E}-15$ & $3 \mathrm{E}+00$ & $7 \mathrm{E}+00$ & $5 \mathrm{E}+00$ & $7 \mathrm{E}-14$ \\
\hline 3 & $2 \mathrm{E}-18$ & $3 \mathrm{E}+00$ & $5 \mathrm{E}+00$ & $3 \mathrm{E}+00$ & $2 \mathrm{E}-15$ \\
8 & $2 \mathrm{E}-18$ & $3 \mathrm{E}+00$ & $4 \mathrm{E}+00$ & $5 \mathrm{E}+00$ & $2 \mathrm{E}-12$ \\
13 & $2 \mathrm{E}-13$ & $3 \mathrm{E}+00$ & $5 \mathrm{E}+00$ & $2 \mathrm{E}+00$ & $1 \mathrm{E}-06$ \\
\hline $13^{*}$ & $1 \mathrm{E}-18$ & $2 \mathrm{E}+00$ & $2 \mathrm{E}+00$ & $3 \mathrm{E}+00$ & $9 \mathrm{E}-07$ \\
\hline 4 & $8 \mathrm{E}-16$ & $3 \mathrm{E}+00$ & $5 \mathrm{E}+00$ & $4 \mathrm{E}+00$ & $2 \mathrm{E}-15$ \\
9 & $7 \mathrm{E}-19$ & $3 \mathrm{E}+00$ & $6 \mathrm{E}+00$ & $2 \mathrm{E}+00$ & $2 \mathrm{E}-15$ \\
14 & $8 \mathrm{E}-24$ & $2 \mathrm{E}+00$ & $3 \mathrm{E}+00$ & $4 \mathrm{E}+00$ & $1 \mathrm{E}-15$ \\
\hline 5 & $0 \mathrm{E}+00$ & $0 \mathrm{E}+00$ & $0 \mathrm{E}+00$ & $0 \mathrm{E}+00$ & $0 \mathrm{E}+00$ \\
10 & $4 \mathrm{E}-22$ & $1 \mathrm{E}+00$ & $3 \mathrm{E}+00$ & $2 \mathrm{E}+00$ & $5 \mathrm{E}-14$ \\
15 & $5 \mathrm{E}-24$ & $3 \mathrm{E}+00$ & $7 \mathrm{E}+00$ & $3 \mathrm{E}+00$ & $1 \mathrm{E}-11$ \\
\hline 16 & $5 \mathrm{E}-19$ & $3 \mathrm{E}+00$ & $6 \mathrm{E}+00$ & $2 \mathrm{E}+00$ & $2 \mathrm{E}-15$ \\
17 & $1 \mathrm{E}-21$ & $1 \mathrm{E}+00$ & $2 \mathrm{E}+00$ & $2 \mathrm{E}+00$ & $1 \mathrm{E}-15$ \\
18 & $5 \mathrm{E}-14$ & $6 \mathrm{E}+01$ & $4 \mathrm{E}+00$ & $2 \mathrm{E}+00$ & $5 \mathrm{E}-14$ \\
19 & $3 \mathrm{E}-06$ & $4 \mathrm{E}+09$ & $6 \mathrm{E}+00$ & $2 \mathrm{E}+00$ & $3 \mathrm{E}-06$ \\
\hline 20 & $3 \mathrm{E}-16$ & $3 \mathrm{E}+00$ & $7 \mathrm{E}+00$ & $3 \mathrm{E}+00$ & $2 \mathrm{E}-15$ \\
21 & $3 \mathrm{E}-16$ & $3 \mathrm{E}+00$ & $2 \mathrm{E}+00$ & $4 \mathrm{E}+00$ & $2 \mathrm{E}-15$ \\
22 & $3 \mathrm{E}-11$ & $4 \mathrm{E}+04$ & $4 \mathrm{E}+00$ & $2 \mathrm{E}+00$ & $3 \mathrm{E}-11$ \\
23 & $2 \mathrm{E}-10$ & $3 \mathrm{E}+05$ & $4 \mathrm{E}+00$ & $2 \mathrm{E}+00$ & $2 \mathrm{E}-10$ \\
\hline
\end{tabular}


Table 7.2: Computed errors after the reordering using Method 2

\begin{tabular}{|c|ccccc|}
\hline Tag & $\left\|A_{21}, B_{21}\right\|_{F}$ & $E_{A, B}$ & $E_{Q}$ & $E_{Z}$ & $\|(E, F)\|_{F}$ \\
\hline 1 & $3 \mathrm{E}-19$ & $1 \mathrm{E}+00$ & $3 \mathrm{E}+00$ & $3 \mathrm{E}+00$ & $1 \mathrm{E}-11$ \\
6 & $6 \mathrm{E}-22$ & $3 \mathrm{E}+00$ & $3 \mathrm{E}+00$ & $5 \mathrm{E}+00$ & $4 \mathrm{E}-12$ \\
11 & $2 \mathrm{E}-20$ & $2 \mathrm{E}+00$ & $3 \mathrm{E}+00$ & $4 \mathrm{E}+00$ & $6 \mathrm{E}-13$ \\
\hline 2 & $1 \mathrm{E}-15$ & $2 \mathrm{E}+00$ & $8 \mathrm{E}+00$ & $2 \mathrm{E}+00$ & $7 \mathrm{E}-15$ \\
7 & $5 \mathrm{E}-16$ & $2 \mathrm{E}+00$ & $9 \mathrm{E}-01$ & $1 \mathrm{E}+00$ & $6 \mathrm{E}-15$ \\
12 & $1 \mathrm{E}-15$ & $3 \mathrm{E}+00$ & $6 \mathrm{E}+00$ & $3 \mathrm{E}+00$ & $7 \mathrm{E}-14$ \\
\hline 3 & $2 \mathrm{E}-18$ & $3 \mathrm{E}+00$ & $5 \mathrm{E}+00$ & $3 \mathrm{E}+00$ & $2 \mathrm{E}-15$ \\
8 & $1 \mathrm{E}-18$ & $3 \mathrm{E}+00$ & $4 \mathrm{E}+00$ & $5 \mathrm{E}+00$ & $2 \mathrm{E}-12$ \\
13 & $1 \mathrm{E}-04$ & $3 \mathrm{E}+02$ & $4 \mathrm{E}+00$ & $2 \mathrm{E}+00$ & $1 \mathrm{E}-04$ \\
\hline $13^{*}$ & $1 \mathrm{E}-18$ & $2 \mathrm{E}+00$ & $2 \mathrm{E}+00$ & $3 \mathrm{E}+00$ & $9 \mathrm{E}-07$ \\
\hline 4 & $3 \mathrm{E}-16$ & $3 \mathrm{E}+00$ & $4 \mathrm{E}+00$ & $4 \mathrm{E}+00$ & $2 \mathrm{E}-15$ \\
9 & $7 \mathrm{E}-19$ & $2 \mathrm{E}+00$ & $4 \mathrm{E}+00$ & $2 \mathrm{E}+00$ & $2 \mathrm{E}-15$ \\
14 & $6 \mathrm{E}-24$ & $2 \mathrm{E}+00$ & $3 \mathrm{E}+00$ & $4 \mathrm{E}+00$ & $1 \mathrm{E}-15$ \\
\hline 5 & $0 \mathrm{E}+00$ & $0 \mathrm{E}+00$ & $0 \mathrm{E}+00$ & $0 \mathrm{E}+00$ & $0 \mathrm{E}+00$ \\
10 & $4 \mathrm{E}-22$ & $1 \mathrm{E}+00$ & $3 \mathrm{E}+00$ & $2 \mathrm{E}+00$ & $5 \mathrm{E}-14$ \\
15 & $2 \mathrm{E}-24$ & $3 \mathrm{E}+00$ & $7 \mathrm{E}+00$ & $3 \mathrm{E}+00$ & $1 \mathrm{E}-11$ \\
\hline 16 & $6 \mathrm{E}-19$ & $4 \mathrm{E}+00$ & $6 \mathrm{E}+00$ & $2 \mathrm{E}+00$ & $3 \mathrm{E}-15$ \\
17 & $9 \mathrm{E}-22$ & $1 \mathrm{E}+00$ & $2 \mathrm{E}+00$ & $2 \mathrm{E}+00$ & $1 \mathrm{E}-15$ \\
18 & $5 \mathrm{E}-14$ & $6 \mathrm{E}+01$ & $3 \mathrm{E}+00$ & $2 \mathrm{E}+00$ & $5 \mathrm{E}-14$ \\
19 & $3 \mathrm{E}-06$ & $4 \mathrm{E}+09$ & $5 \mathrm{E}+00$ & $1 \mathrm{E}+00$ & $3 \mathrm{E}-06$ \\
\hline 20 & $1 \mathrm{E}-16$ & $3 \mathrm{E}+00$ & $6 \mathrm{E}+00$ & $4 \mathrm{E}+00$ & $2 \mathrm{E}-15$ \\
21 & $2 \mathrm{E}-16$ & $3 \mathrm{E}+00$ & $2 \mathrm{E}+00$ & $5 \mathrm{E}+00$ & $3 \mathrm{E}-15$ \\
22 & $6 \mathrm{E}-12$ & $7 \mathrm{E}+03$ & $3 \mathrm{E}+00$ & $3 \mathrm{E}+00$ & $6 \mathrm{E}-12$ \\
23 & $2 \mathrm{E}-10$ & $3 \mathrm{E}+05$ & $2 \mathrm{E}+00$ & $2 \mathrm{E}+00$ & $2 \mathrm{E}-10$ \\
\hline
\end{tabular}


Table 7.3: Method 2 - Computed stability test values and tolerances

\begin{tabular}{|c|cccc|}
\hline Tag & Weak $(B=Q R)$ & Weak $(B=R Q)$ & Strong & tol $_{1,2}$ \\
\hline 1 & $8.17 \mathrm{E}-19$ & $3.16 \mathrm{E}-19$ & $8.53 \mathrm{E}-12$ & $7.02 \mathrm{E}-11$ \\
6 & $7.03 \mathrm{E}-22$ & $1.05 \mathrm{E}-21$ & $3.03 \mathrm{E}-12$ & $1.38 \mathrm{E}-11$ \\
11 & $2.41 \mathrm{E}-19$ & $2.55 \mathrm{E}-21$ & $6.544 \mathrm{E}-13$ & $3.59 \mathrm{E}-12$ \\
\hline 2 & $9.75 \mathrm{E}-16$ & $3.88 \mathrm{E}-13$ & $6.56 \mathrm{E}-15$ & $3.06 \mathrm{E}-14$ \\
7 & $3.19 \mathrm{E}-16$ & $4.75 \mathrm{E}-08$ & $3.57 \mathrm{E}-15$ & $3.06 \mathrm{E}-14$ \\
12 & $1.58 \mathrm{E}-15$ & $3.99 \mathrm{E}-6$ & $4.92 \mathrm{E}-14$ & $3.06 \mathrm{E}-14$ \\
\hline 3 & $6.97 \mathrm{E}-19$ & $7.27 \mathrm{E}-19$ & $1.60 \mathrm{E}-15$ & $7.94 \mathrm{E}-15$ \\
8 & $5.26 \mathrm{E}-19$ & $8.56 \mathrm{E}-19$ & $9.49 \mathrm{E}-13$ & $4.44 \mathrm{E}-12$ \\
13 & $1.20 \mathrm{E}-04$ & $1.20 \mathrm{E}-04$ & $1.20 \mathrm{E}-04$ & $4.44 \mathrm{E}-06$ \\
\hline $13^{*}$ & $6.09 \mathrm{E}-19$ & $9.02 \mathrm{E}-19$ & $7.64 \mathrm{E}-07$ & $4.44 \mathrm{E}-06$ \\
\hline 4 & $1.41 \mathrm{E}-16$ & $2.20 \mathrm{E}-16$ & $1.45 \mathrm{E}-15$ & $6.75 \mathrm{E}-15$ \\
9 & $1.33 \mathrm{E}-19$ & $1.33 \mathrm{E}-19$ & $1.36 \mathrm{E}-15$ & $6.58 \mathrm{E}-15$ \\
14 & $5.91 \mathrm{E}-25$ & $5.91 \mathrm{E}-25$ & $8.83 \mathrm{E}-16$ & $6.58 \mathrm{E}-15$ \\
\hline 5 & $0.00 \mathrm{E}+00$ & $0.00 \mathrm{E}+00$ & $0.00 \mathrm{E}+00$ & $6.28 \mathrm{E}-15$ \\
10 & $2.38 \mathrm{E}-22$ & $2.38 \mathrm{E}-22$ & $5.97 \mathrm{E}-14$ & $3.93 \mathrm{E}-13$ \\
15 & $1.41 \mathrm{E}-24$ & $1.41 \mathrm{E}-24$ & $1.09 \mathrm{E}-11$ & $3.93 \mathrm{E}-11$ \\
\hline 16 & $7.82 \mathrm{E}-17$ & $7.85 \mathrm{E}-17$ & $1.25 \mathrm{E}-15$ & $6.28 \mathrm{E}-15$ \\
17 & $7.85 \mathrm{E}-17$ & $7.85 \mathrm{E}-17$ & $7.71 \mathrm{E}-16$ & $6.28 \mathrm{E}-15$ \\
18 & $4.62 \mathrm{E}-14$ & $4.62 \mathrm{E}-14$ & $4.62 \mathrm{E}-14$ & $8.01 \mathrm{E}-15$ \\
19 & $3.05 \mathrm{E}-06$ & $3.05 \mathrm{E}-06$ & $3.05 \mathrm{E}-06$ & $8.01 \mathrm{E}-15$ \\
\hline 20 & $2.94 \mathrm{E}-16$ & $4.14 \mathrm{E}-16$ & $1.88 \mathrm{E}-15$ & $5.91 \mathrm{E}-15$ \\
21 & $1.95 \mathrm{E}-16$ & $4.22 \mathrm{E}-18$ & $1.17 \mathrm{E}-15$ & $5.88 \mathrm{E}-15$ \\
22 & $5.73 \mathrm{E}-12$ & $5.79 \mathrm{E}-12$ & $5.73 \mathrm{E}-12$ & $8.01 \mathrm{E}-15$ \\
23 & $2.38 \mathrm{E}-10$ & $2.38 \mathrm{E}-10$ & $2.38 \mathrm{E}-10$ & $8.01 \mathrm{E}-15$ \\
\hline
\end{tabular}


Table 7.4: Computed errors after the reordering using Method 3

\begin{tabular}{|c|ccccc|}
\hline Tag & $\left\|A_{21}, B_{21}\right\|_{F}$ & $E_{A, B}$ & $E_{Q}$ & $E_{Z}$ & $\|(E, F)\|_{F}$ \\
\hline 1 & $7 \mathrm{E}-19$ & $5 \mathrm{E}+00$ & $5 \mathrm{E}+00$ & $3 \mathrm{E}+00$ & $3 \mathrm{E}-11$ \\
6 & $4 \mathrm{E}-22$ & $1 \mathrm{E}+00$ & $6 \mathrm{E}+00$ & $3 \mathrm{E}+00$ & $1 \mathrm{E}-12$ \\
11 & $3 \mathrm{E}-20$ & $3 \mathrm{E}+00$ & $4 \mathrm{E}+00$ & $3 \mathrm{E}+00$ & $9 \mathrm{E}-13$ \\
\hline 2 & $5 \mathrm{E}-16$ & $4 \mathrm{E}+00$ & $8 \mathrm{E}+00$ & $5 \mathrm{E}+00$ & $1 \mathrm{E}-14$ \\
7 & $5 \mathrm{E}-16$ & $9 \mathrm{E}-01$ & $4 \mathrm{E}+00$ & $1 \mathrm{E}+00$ & $3 \mathrm{E}-15$ \\
12 & $1 \mathrm{E}-15$ & $4 \mathrm{E}+00$ & $4 \mathrm{E}+00$ & $3 \mathrm{E}+00$ & $1 \mathrm{E}-14$ \\
\hline 3 & $9 \mathrm{E}-19$ & $3 \mathrm{E}+00$ & $2 \mathrm{E}+00$ & $5 \mathrm{E}+00$ & $3 \mathrm{E}-15$ \\
8 & $9 \mathrm{E}-19$ & $5 \mathrm{E}+00$ & $6 \mathrm{E}+00$ & $4 \mathrm{E}+00$ & $2 \mathrm{E}-12$ \\
13 & $1 \mathrm{E}-07$ & $4 \mathrm{E}+00$ & $1 \mathrm{E}+01$ & $4 \mathrm{E}+00$ & $2 \mathrm{E}-06$ \\
\hline 4 & $2 \mathrm{E}-16$ & $4 \mathrm{E}+00$ & $7 \mathrm{E}+00$ & $4 \mathrm{E}+00$ & $3 \mathrm{E}-15$ \\
9 & $1 \mathrm{E}-18$ & $2 \mathrm{E}+00$ & $6 \mathrm{E}+00$ & $3 \mathrm{E}+00$ & $1 \mathrm{E}-15$ \\
14 & $5 \mathrm{E}-24$ & $3 \mathrm{E}+00$ & $5 \mathrm{E}+00$ & $2 \mathrm{E}+00$ & $2 \mathrm{E}-15$ \\
\hline 5 & $0 \mathrm{E}+00$ & $0 \mathrm{E}+00$ & $0 \mathrm{E}+00$ & $0 \mathrm{E}+00$ & $0 \mathrm{E}+00$ \\
10 & $5 \mathrm{E}-22$ & $2 \mathrm{E}+00$ & $4 \mathrm{E}+00$ & $1 \mathrm{E}+00$ & $8 \mathrm{E}-14$ \\
15 & $3 \mathrm{E}-24$ & $4 \mathrm{E}+00$ & $5 \mathrm{E}+00$ & $2 \mathrm{E}+00$ & $2 \mathrm{E}-11$ \\
\hline 16 & $1 \mathrm{E}-18$ & $4 \mathrm{E}+00$ & $7 \mathrm{E}+00$ & $1 \mathrm{E}+00$ & $3 \mathrm{E}-15$ \\
17 & $2 \mathrm{E}-16$ & $2 \mathrm{E}+00$ & $2 \mathrm{E}+00$ & $3 \mathrm{E}+00$ & $2 \mathrm{E}-15$ \\
18 & $3 \mathrm{E}-16$ & $2 \mathrm{E}+00$ & $2 \mathrm{E}+00$ & $3 \mathrm{E}+00$ & $2 \mathrm{E}-15$ \\
19 & $2 \mathrm{E}-18$ & $5 \mathrm{E}+00$ & $8 \mathrm{E}+00$ & $1 \mathrm{E}+01$ & $4 \mathrm{E}-15$ \\
\hline 20 & $9 \mathrm{E}-17$ & $4 \mathrm{E}+00$ & $5 \mathrm{E}+00$ & $5 \mathrm{E}+00$ & $3 \mathrm{E}-15$ \\
21 & $2 \mathrm{E}-16$ & $3 \mathrm{E}+00$ & $4 \mathrm{E}+00$ & $5 \mathrm{E}+00$ & $3 \mathrm{E}-15$ \\
22 & $2 \mathrm{E}-16$ & $3 \mathrm{E}+00$ & $5 \mathrm{E}+00$ & $2 \mathrm{E}+00$ & $2 \mathrm{E}-15$ \\
23 & $4 \mathrm{E}-16$ & $1 \mathrm{E}+01$ & $2 \mathrm{E}+01$ & $8 \mathrm{E}+00$ & $8 \mathrm{E}-15$ \\
\hline
\end{tabular}


Table 7.5: Computed errors after the reordering using Algorithm 590

\begin{tabular}{|c|ccccc|}
\hline Tag & $\left\|A_{21}, B_{21}\right\|_{F}$ & $E_{A, B}$ & $E_{Q}$ & $E_{Z}$ & $\|(E, F)\|_{F}$ \\
\hline 1 & $2 \mathrm{E}-12$ & $3 \mathrm{E}+00$ & $5 \mathrm{E}+00$ & $2 \mathrm{E}+00$ & $2 \mathrm{E}-11$ \\
6 & $2 \mathrm{E}-13$ & $2 \mathrm{E}+00$ & $5 \mathrm{E}+00$ & $3 \mathrm{E}+00$ & $2 \mathrm{E}-12$ \\
11 & $2 \mathrm{E}-13$ & $6 \mathrm{E}+00$ & $1 \mathrm{E}+01$ & $8 \mathrm{E}+00$ & $2 \mathrm{E}-12$ \\
\hline 2 & $8 \mathrm{E}-16$ & $5 \mathrm{E}+00$ & $6 \mathrm{E}+00$ & $4 \mathrm{E}+00$ & $2 \mathrm{E}-14$ \\
7 & $1 \mathrm{E}-15$ & $6 \mathrm{E}+00$ & $5 \mathrm{E}+00$ & $5 \mathrm{E}+00$ & $2 \mathrm{E}-14$ \\
12 & $3 \mathrm{E}-15$ & $7 \mathrm{E}+00$ & $7 \mathrm{E}+00$ & $3 \mathrm{E}+00$ & $2 \mathrm{E}-14$ \\
\hline 3 & $2 \mathrm{E}-16$ & $3 \mathrm{E}+00$ & $4 \mathrm{E}+00$ & $4 \mathrm{E}+00$ & $2 \mathrm{E}-15$ \\
8 & $1 \mathrm{E}-13$ & $2 \mathrm{E}+00$ & $3 \mathrm{E}+00$ & $3 \mathrm{E}+00$ & $7 \mathrm{E}-13$ \\
13 & $4 \mathrm{E}-07$ & $7 \mathrm{E}+00$ & $6 \mathrm{E}+00$ & $1 \mathrm{E}+01$ & $3 \mathrm{E}-06$ \\
\hline 4 & $5 \mathrm{E}-16$ & $3 \mathrm{E}+00$ & $3 \mathrm{E}+00$ & $4 \mathrm{E}+00$ & $2 \mathrm{E}-15$ \\
9 & $3 \mathrm{E}-16$ & $3 \mathrm{E}+00$ & $4 \mathrm{E}+00$ & $4 \mathrm{E}+00$ & $2 \mathrm{E}-15$ \\
14 & $6 \mathrm{E}-16$ & $3 \mathrm{E}+00$ & $3 \mathrm{E}+00$ & $3 \mathrm{E}+00$ & $2 \mathrm{E}-15$ \\
\hline 5 & $3 \mathrm{E}-16$ & $4 \mathrm{E}+00$ & $6 \mathrm{E}+00$ & $4 \mathrm{E}+00$ & $2 \mathrm{E}-15$ \\
10 & $1 \mathrm{E}-14$ & $3 \mathrm{E}+00$ & $6 \mathrm{E}+00$ & $4 \mathrm{E}+00$ & $1 \mathrm{E}-13$ \\
15 & $2 \mathrm{E}-12$ & $2 \mathrm{E}+00$ & $1 \mathrm{E}+00$ & $2 \mathrm{E}+00$ & $8 \mathrm{E}-12$ \\
\hline 16 & $3 \mathrm{E}-19$ & $2 \mathrm{E}+00$ & $2 \mathrm{E}+00$ & $3 \mathrm{E}+00$ & $2 \mathrm{E}-15$ \\
17 & $3 \mathrm{E}-22$ & $3 \mathrm{E}+00$ & $3 \mathrm{E}+00$ & $3 \mathrm{E}+00$ & $2 \mathrm{E}-15$ \\
18 & $5 \mathrm{E}-16$ & $4 \mathrm{E}+00$ & $5 \mathrm{E}+00$ & $5 \mathrm{E}+00$ & $3 \mathrm{E}-15$ \\
19 & $5 \mathrm{E}-16$ & $4 \mathrm{E}+00$ & $6 \mathrm{E}+00$ & $5 \mathrm{E}+00$ & $3 \mathrm{E}-15$ \\
\hline 20 & $2 \mathrm{E}-16$ & $1 \mathrm{E}+00$ & $2 \mathrm{E}+00$ & $1 \mathrm{E}+00$ & $1 \mathrm{E}-15$ \\
21 & $1 \mathrm{E}-16$ & $1 \mathrm{E}+00$ & $2 \mathrm{E}+00$ & $2 \mathrm{E}+00$ & $1 \mathrm{E}-15$ \\
22 & $2 \mathrm{E}-16$ & $3 \mathrm{E}+00$ & $4 \mathrm{E}+00$ & $3 \mathrm{E}+00$ & $2 \mathrm{E}-15$ \\
23 & $4 \mathrm{E}-16$ & $4 \mathrm{E}+01$ & $4 \mathrm{E}+01$ & $4 \mathrm{E}+01$ & $3 \mathrm{E}-14$ \\
\hline
\end{tabular}


Table 7.6: Method 2 - Some computed quantities before and after the reordering

\begin{tabular}{|c|c|ccc|cc|}
\hline Tag & Dif $_{u}$-before & Dif $_{u}$-after & Dif $_{u} /$ DIF $(1)-\mathrm{F}$ & Dif $_{u} /$ DIF $(1)-1$ & Dif $_{l}$ (DTGSNA $)$ & Dif $_{l}$ (ZTGSNA $)$ \\
\hline 1 & $1 \mathrm{E}-02$ & $1 \mathrm{E}-02$ & 0.57 & 1.90 & $3 \mathrm{E}-2$ & $3 \mathrm{E}-4$ \\
6 & $3 \mathrm{E}-04$ & $3 \mathrm{E}-06$ & 0.63 & 1.30 & $4 \mathrm{E}-4$ & $1 \mathrm{E}-6$ \\
11 & $1 \mathrm{E}-07$ & $1 \mathrm{E}-07$ & 0.96 & 1.20 & $2 \mathrm{E}-7$ & $3 \mathrm{E}-6$ \\
\hline 2 & $7 \mathrm{E}-01$ & $1 \mathrm{E}-01$ & 0.52 & 1.00 & $1 \mathrm{E}-3$ & $2 \mathrm{E}-3$ \\
7 & $7 \mathrm{E}-01$ & $1 \mathrm{E}-01$ & 0.52 & 1.00 & $1 \mathrm{E}-9$ & $2 \mathrm{E}-9$ \\
12 & $7 \mathrm{E}-01$ & $1 \mathrm{E}-02$ & 0.52 & 1.00 & $1 \mathrm{E}-15$ & $2 \mathrm{E}-15$ \\
\hline 3 & $4 \mathrm{E}-04$ & $4 \mathrm{E}-04$ & 0.87 & 1.60 & $7 \mathrm{E}-4$ & $7 \mathrm{E}-4$ \\
8 & $7 \mathrm{E}-07$ & $7 \mathrm{E}-07$ & 0.71 & 1.40 & $1 \mathrm{E}-6$ & $2 \mathrm{E}-6$ \\
13 & $8 \mathrm{E}-09$ & $2 \mathrm{E}-07$ & 0.73 & 1.50 & $1 \mathrm{E}-7$ & $4 \mathrm{E}-7$ \\
\hline $13^{*}$ & $8 \mathrm{E}-09$ & $2 \mathrm{E}-10$ & $2 \mathrm{E}+02$ & $4 \mathrm{E}+02$ & - & - \\
\hline 4 & $5 \mathrm{E}-02$ & $4 \mathrm{E}-03$ & 0.35 & 1.30 & $9 \mathrm{E}-2$ & $2 \mathrm{E}-2$ \\
9 & $5 \mathrm{E}-05$ & $2 \mathrm{E}-06$ & 0.19 & 1.10 & $9 \mathrm{E}-5$ & $2 \mathrm{E}-8$ \\
14 & $5 \mathrm{E}-10$ & $2 \mathrm{E}-10$ & 0.19 & 1.10 & 0 & 0 \\
\hline 5 & $5 \mathrm{E}-06$ & $5 \mathrm{E}-06$ & 0.55 & 1.40 & $9 \mathrm{E}-6$ & $7 \mathrm{E}-6$ \\
10 & $5 \mathrm{E}-06$ & $2 \mathrm{E}-07$ & 0.19 & 1.10 & $9 \mathrm{E}-6$ & $1 \mathrm{E}-12$ \\
15 & $5 \mathrm{E}-06$ & $2 \mathrm{E}-07$ & 0.19 & 1.10 & $9 \mathrm{E}-6$ & $1 \mathrm{E}-14$ \\
\hline 16 & $4 \mathrm{E}-10$ & $4 \mathrm{E}-10$ & 0.35 & 1.40 & $3 \mathrm{E}-22$ & $4 \mathrm{E}-22$ \\
17 & $2 \mathrm{E}-16$ & $2 \mathrm{E}-17$ & 20.00 & 79.00 & $3 \mathrm{E}-28$ & $4 \mathrm{E}-28$ \\
18 & $4 \mathrm{E}-10$ & $4 \mathrm{E}-10$ & 0.42 & 1.60 & $6 \mathrm{E}-10$ & $3 \mathrm{E}-13$ \\
19 & $5 \mathrm{E}-18$ & $6 \mathrm{E}-16$ & 0.35 & 1.40 & $8 \mathrm{E}-16$ & $3 \mathrm{E}-19$ \\
\hline 20 & $4 \mathrm{E}-10$ & $3 \mathrm{E}-06$ & 0.71 & 1.50 & $3 \mathrm{E}-16$ & $3 \mathrm{E}-16$ \\
21 & $2 \mathrm{E}-16$ & $3 \mathrm{E}-12$ & 0.71 & 1.50 & $3 \mathrm{E}-16$ & $3 \mathrm{E}-16$ \\
22 & $4 \mathrm{E}-10$ & $3 \mathrm{E}-06$ & 0.71 & 1.50 & $2 \mathrm{E}-8$ & $2 \mathrm{E}-7$ \\
23 & $2 \mathrm{E}-16$ & $4 \mathrm{E}-17$ & 44.00 & 100.00 & $1 \mathrm{E}-16$ & $3 \mathrm{E}-16$ \\
\hline
\end{tabular}




\section{A Calling sequence DTGEXC}

Here we display the parameter list and the leading comment lines of the double precision routine DTGEXC.

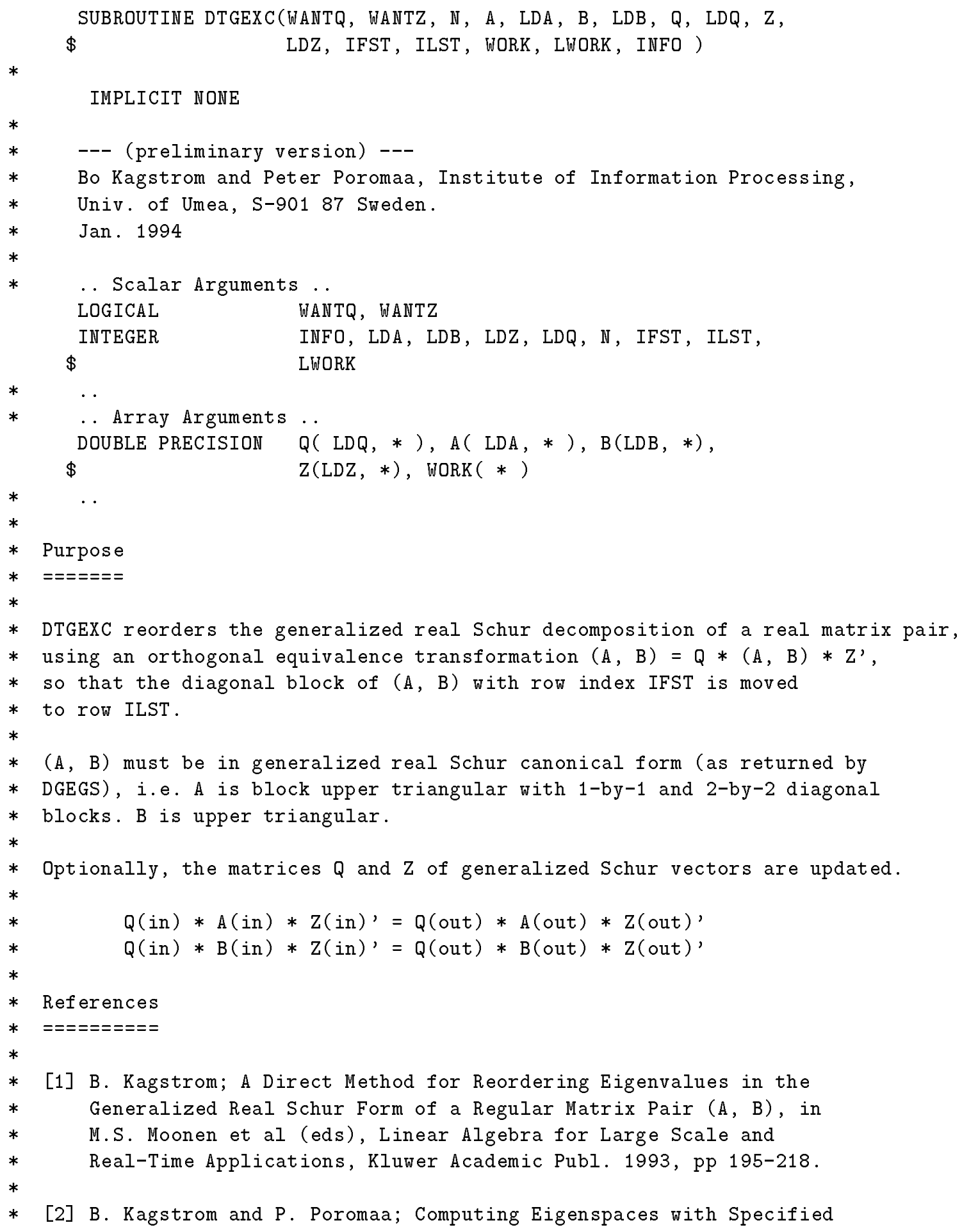


Eigenvalues of a Regular Matrix Pair (A, B) and Condition Estimation: Theory, Algorithms and Software, Report UMINF - 94.04, Inst. of Information Processing, University of Umea, S-901 87 Umea, Sweden, Febrauary 1994. (also published as LAPACK Working Note xx)

[3] B. Kagstrom and P. Poromaa, LAPACK-Style Algorithms and Software for Solving the Generalized Sylvester Equation and Estimating the Separation between Regular Matrix Pairs, Report UMINF - 93.23, Inst. of Information Processing, University of Umea, S-901 87 Umea, Sweden, November 1993. (also published as LAPACK Working Note $\mathrm{xx}$ )

Arguments

$========$

WANTQ (input) LOGICAL

.TRUE. : update the left transformation matrix $Q$;

.FALSE. : do not update $Q$.

WANTZ (input) LOGICAL

.TRUE. : update the right transformation matrix $\mathrm{z}$;

.FALSE. : do not update $\mathrm{Z}$.

(input) INTEGER

The order of the matrices $A$ and $B . \mathbb{N}>=0$.

A, B (input/output) DOUBLE PRECISION arrays, dimensions (LDA(B),N) On entry, the matrix pair (A, B), in generalized real Schur canonical form.

On exit, the updated matrix pair (A, B), again in generalized real Schur canonical form.

LDA (input) INTEGER

The leading dimension of the array $A$. LDA $>=\max (1, N)$.

(input) INTEGER

The leading dimension of the array $B . L D B ~>=\max (1, N)$.

(input/output) DOUBLE PRECISION array, dimension (LDZ,N)

On entry, if WANTQ is .TRUE., the orthogonal matrix $Q$.

On exit, if WANTQ is .TRUE., the updated matrix $Q$.

If WANTQ is .FALSE., $Q$ is not referenced.

(input) INTEGER

The leading dimension of the array $Q$.

LDQ $>=1$; and if WANTQ is .TRUE., LDQ $>=\mathbb{N}$.

(input/output) DOUBLE PRECISION array, dimension (LDZ,N)

On entry, if WANTZ is .TRUE., the orthogonal matrix $Z$.

On exit, if WANTZ is .TRUE., the updated matrix $Z$.

If WANTZ is .FALSE., $Z$ is not referenced. 


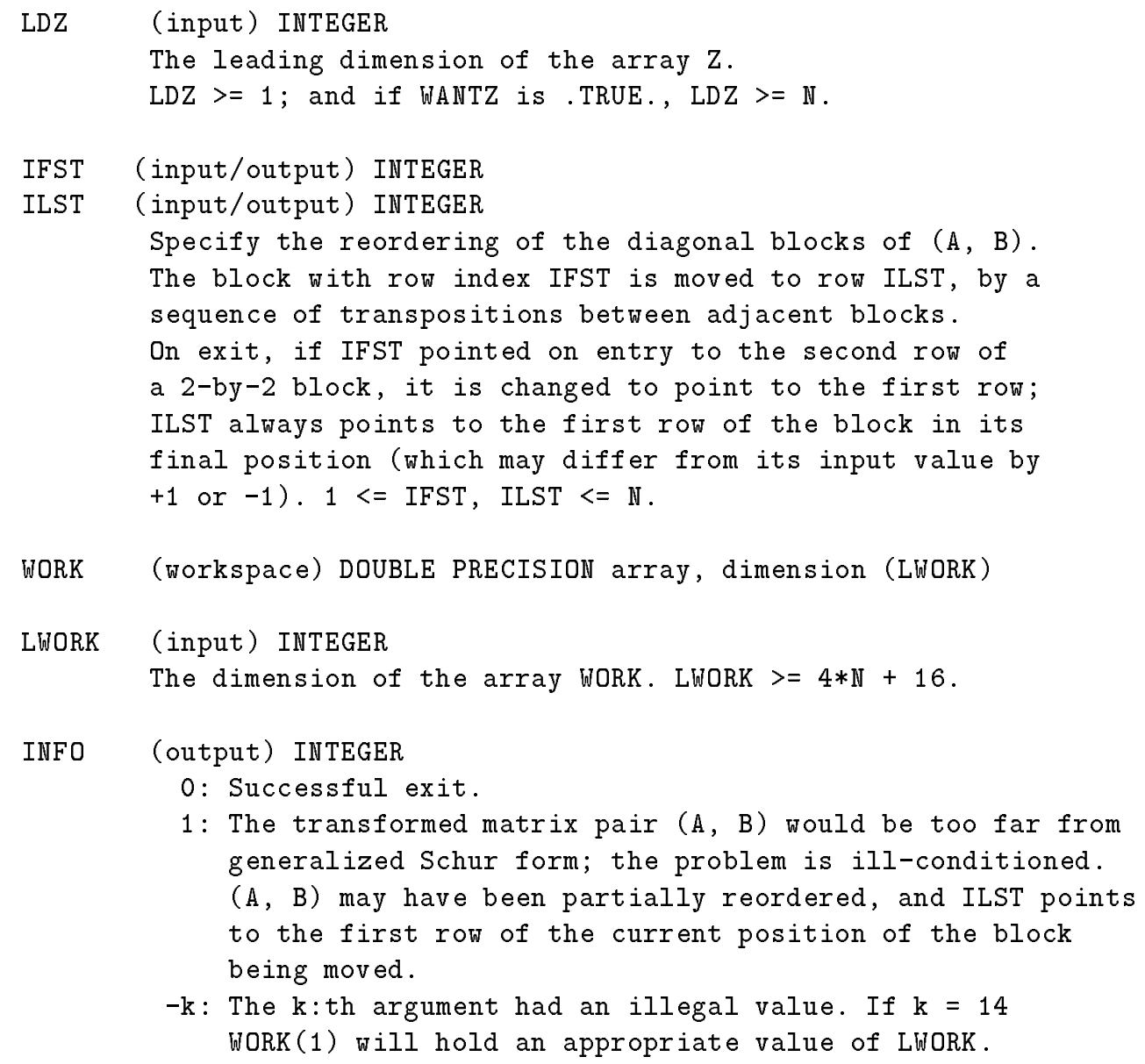

\section{B Calling sequence DTGEX2}

Here we display the parameter list and the leading comment lines of the double precision routine DTGEX2.

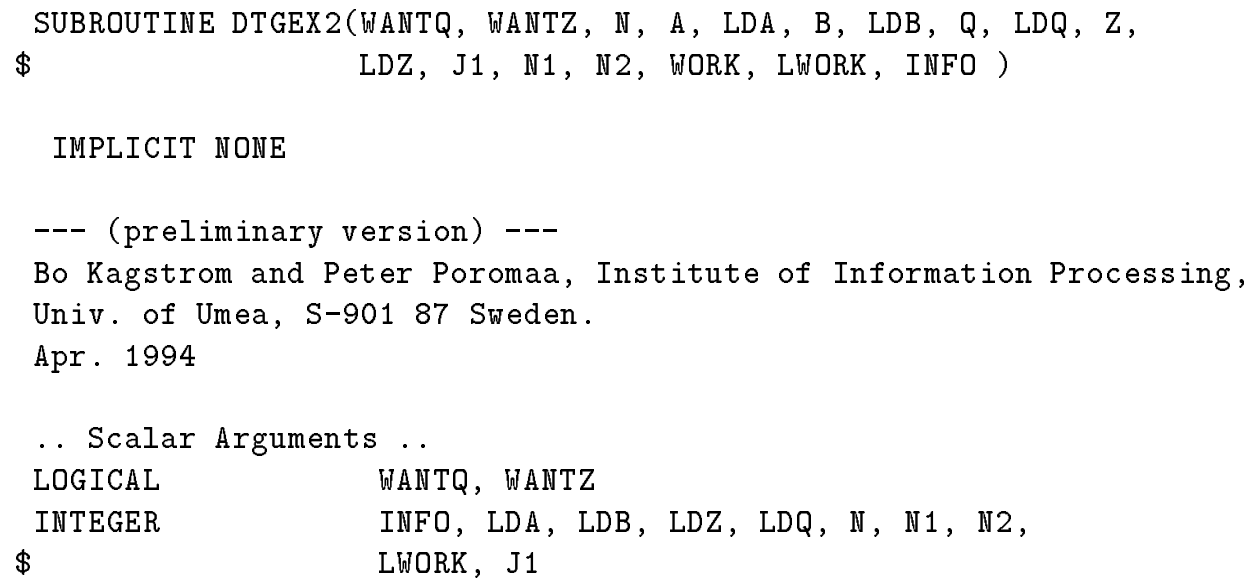




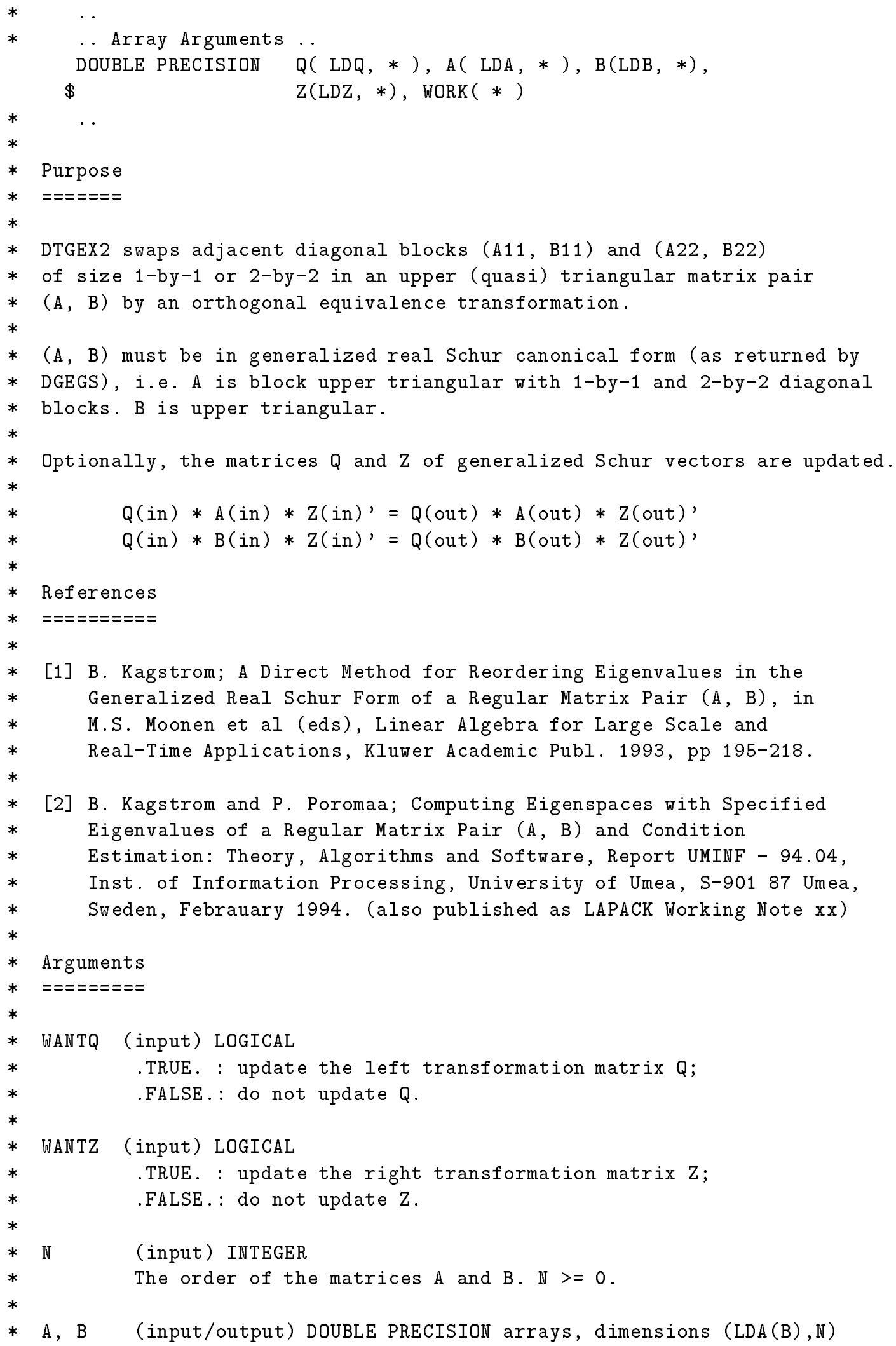




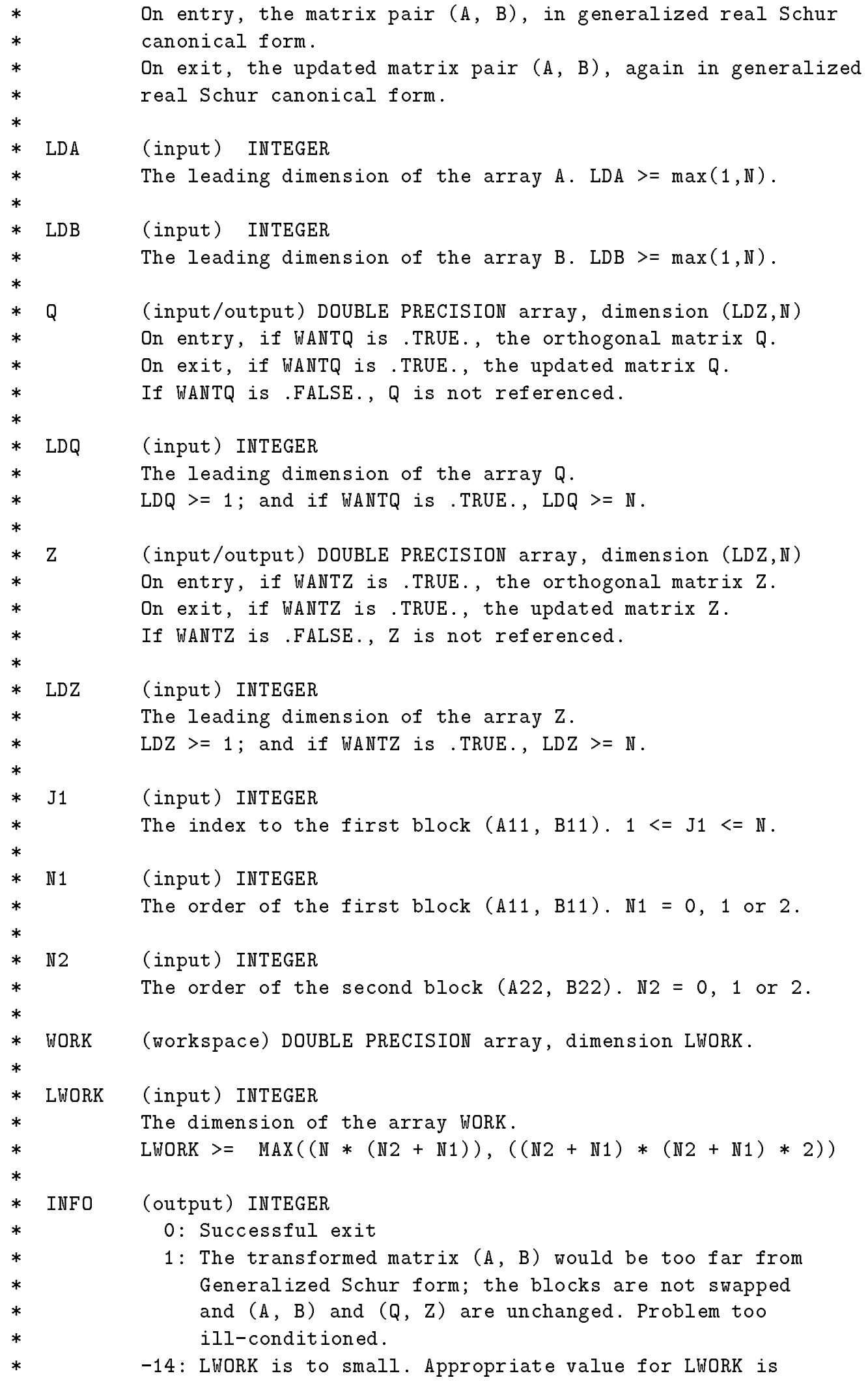

* J1 (input) INTEGER canonical form.

On exit, the updated matrix pair (A, B), again in generalized real Schur canonical form.

The leading dimension of the array $B . \operatorname{LDB}>=\max (1, \mathbb{N})$.

(input/output) DOUBLE PRECISION array, dimension (LDZ,N)

On entry, if WANTQ is .TRUE., the orthogonal matrix $Q$.

On exit, if WANTQ is .TRUE., the updated matrix $Q$.

If WANTQ is .FALSE., $Q$ is not referenced.

The leading dimension of the array $Q$.

LDQ $>=1$; and if WANTQ is . TRUE., LDQ $>=\mathbb{N}$.

(input/output) DOUBLE PRECISION array, dimension (LDZ,N)

On entry, if WANTZ is .TRUE., the orthogonal matrix $Z$.

On exit, if WANTZ is .TRUE., the updated matrix $Z$.

If WANTZ is .FALSE., $Z$ is not referenced.

LDZ (input) INTEGER

The leading dimension of the array $\mathrm{Z}$.

LDZ $>=1$; and if WANTZ is . TRUE., LDZ $>=N$.

The index to the first block (A11, B11). $1<=\mathrm{J} 1<=\mathrm{N}$.

N1 (input) INTEGER

The order of the first block $(A 11, B 11)$. N1 $=0,1$ or 2 .

N2 (input) INTEGER

The order of the second block $(\mathrm{A} 22, \mathrm{~B} 22)$. N2 $=0,1$ or 2 .

WORK (workspace) DOUBLE PRECISION array, dimension LWORK.

LWORK (input) INTEGER

The dimension of the array WORK.

LWORK $>=\operatorname{MAX}((\mathbb{N} *(\mathbb{N} 2+\mathbb{N} 1)),((\mathbb{N} 2+\mathbb{N} 1) *(\mathbb{N} 2+\mathbb{N} 1) * 2))$

(output) INTEGER

0 : Successful exit

1: The transformed matrix (A, B) would be too far from Generalized Schur form; the blocks are not swapped and $(A, B)$ and $(Q, Z)$ are unchanged. Problem too ill-conditioned.

-14: LWORK is to small. Appropriate value for LwORK is 


\section{Calling sequence DTGSEN}

Here we display the parameter list and the leading comment lines of the double precision routine DTGSEN.

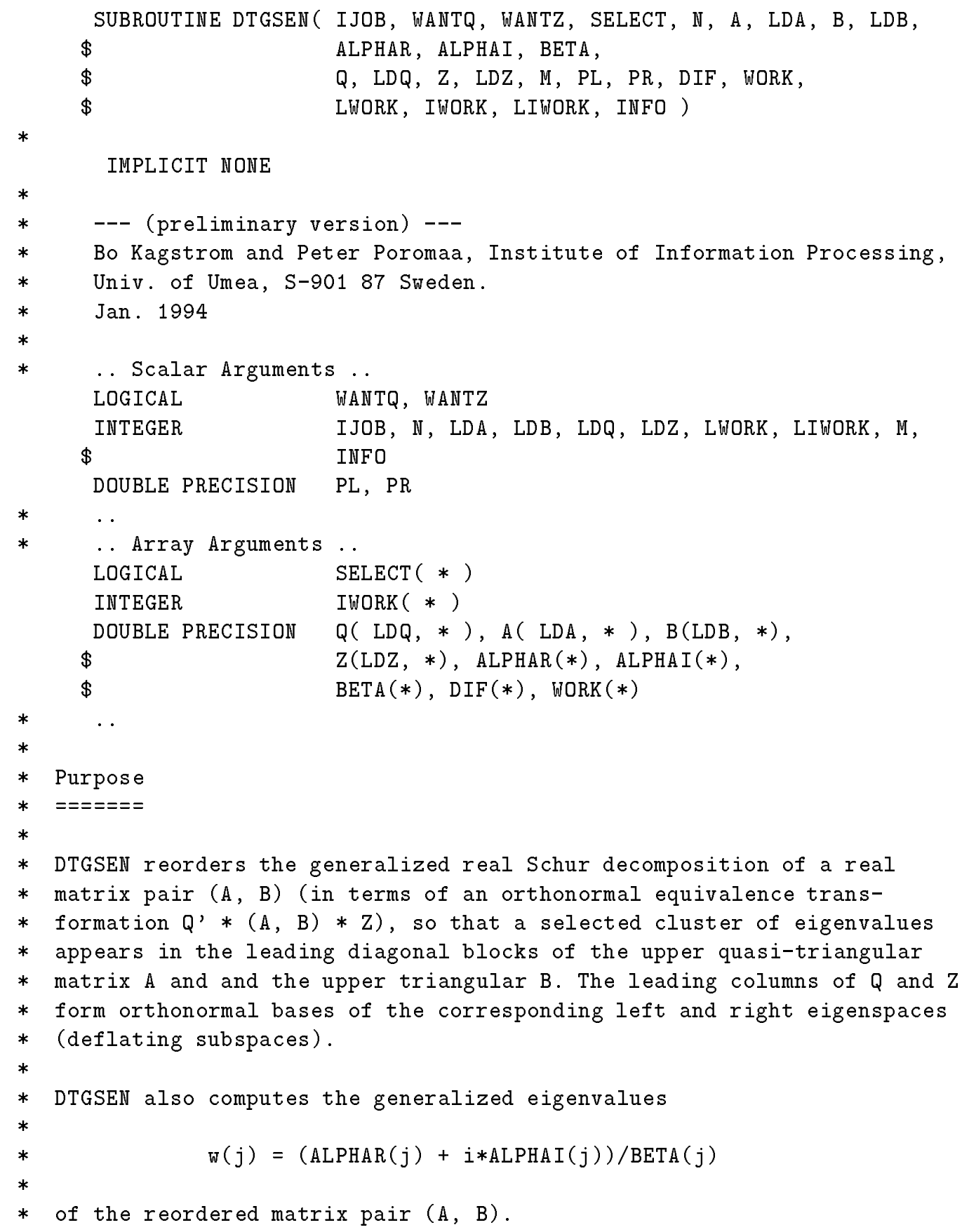


(A, B) must be in generalized real Schur canonical form (as returned by

DGEGS), i.e. A is block upper triangular with 1-by-1 and 2-by-2 diagonal

blocks. B is upper triangular.

Optionally, the matrices $Q$ and $Z$ of generalized Schur vectors are updated.

$Q$ (in) * $A($ in $) * Z(\text { in })^{\prime}=Q$ (out) $* A$ (out) $* Z$ (out)'

$\mathrm{Q}$ (in) * $\mathrm{B}($ in $) * \mathrm{Z}$ (in)' ${ }^{\prime}=\mathrm{Q}$ (out) * $\mathrm{B}$ (out) * $\mathrm{Z}$ (out)'

Optionally, the routine computes estimates of reciprocal condition numbers

for eigenvalues and eigenspaces. These are Difu[(A11,B11), (A22,B22)]

and $\operatorname{Difl}[(A 11, B 11),(A 22, B 22)]$, i.e. the separation(s) between the matrix

pairs (A11, B11) and (A22,B22) that correspond to the selected cluster and

the eigenvalues outside the cluster, respectively, and norms of "projections"

onto left and right eigenspaces w.r.t. the selected cluster in the (1,1)-block.

References

$=========$

[1] B. Kagstrom; A Direct Method for Reordering Eigenvalues in the Generalized Real Schur Form of a Regular Matrix Pair (A, B), in M.S. Moonen et al (eds), Linear Algebra for Large Scale and Real-Time Applications, Kluwer Academic Publ. 1993, pp 195-218.

[2] B. Kagstrom and P. Poromaa; Computing Eigenspaces with Specified Eigenvalues of a Regular Matrix Pair (A, B) and Condition Estimation: Theory, Algorithms and Software, Report UMINF - 94.04, Inst. of Information Processing, University of Umea, S-901 87 Umea, Sweden, February 1994. (also published as LAPACK Working Note xx)

[3] B. Kagstrom and P. Poromaa, LAPACK-Style Algorithms and Software for Solving the Generalized Sylvester Equation and Estimating the Separation between Regular Matrix Pairs, Report UMINF - 93.23, Inst. of Information Processing, University of Umea, S-901 87 Umea, Sweden, November 1993. (also published as LAPACK Working Note $\mathrm{xx}$ )

Arguments

=ニニニニニニ=ニ

I JOB (input) integer

Specifies what functionality is to be obtained.

0 : Only reorder w.r.t. SELECT. No extras.

1 : Reciprocal of norms of "projections" onto left and right eigenspaces w.r.t. the selected cluster (PL and PR).

2 : Upper bounds on Difu and Difl. F-norm-based estimate (DIF(1:2)).

3 : Estimate of Difu and Difl. 1-norm-based estimate (DIF(1:2)). About 5 times as expensive as $I J O B=2$.

4 : Compute PL, PR and DIF (i.e. 0, 1 and 2 above): Economic-version to get it all.

5 : Compute PL, PR and DIF (i.e. 0,1 and 3 above) 


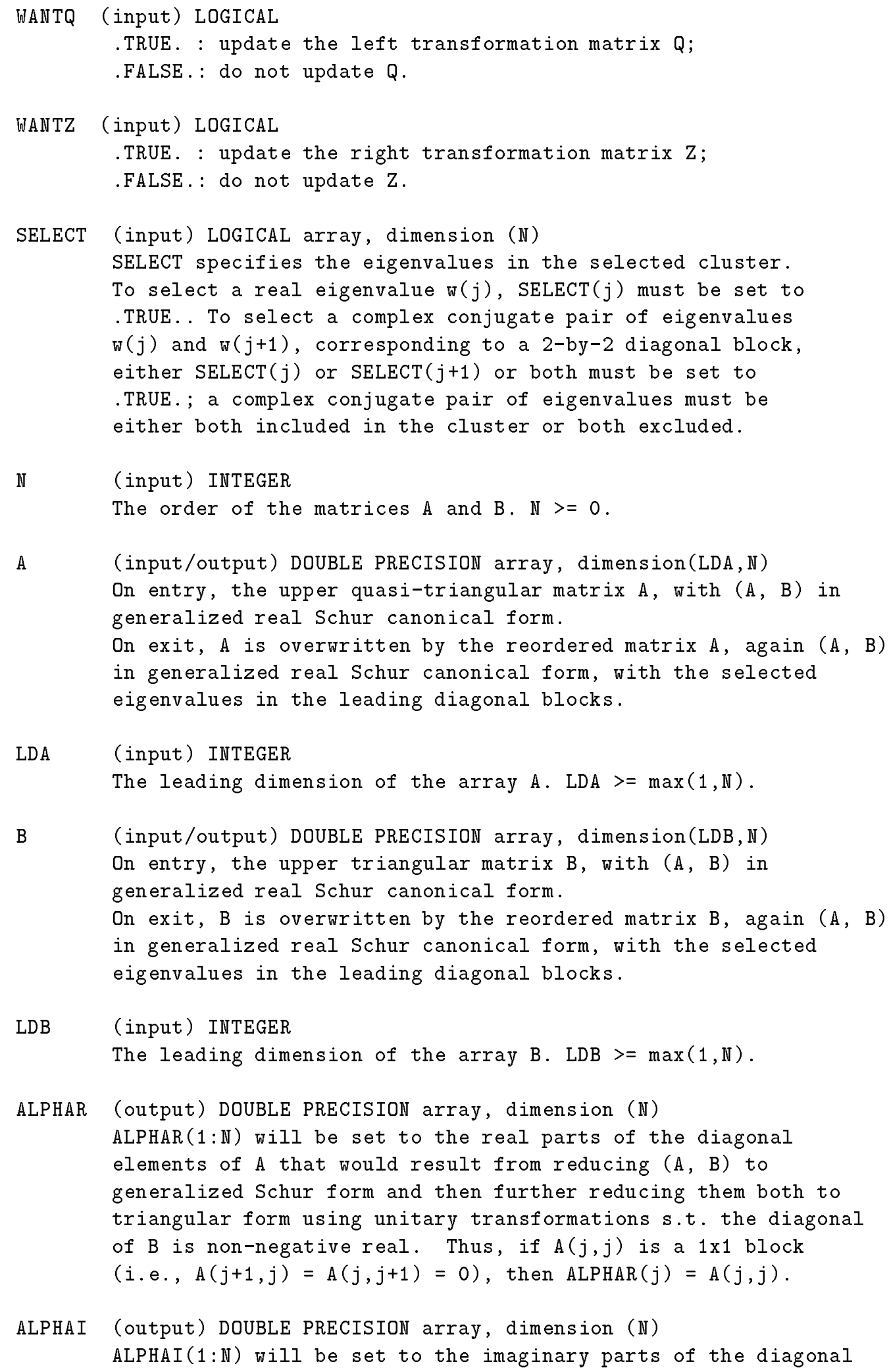


elements of A that would result from reducing (A, B) to generalized Schur form and then further reducing them both to triangular form using unitary transformations s.t. the diagonal of $B$ is non-negative real. Thus, if $A(j, j)$ is a $1 \times 1 \mathrm{block}$ (i.e., $A(j+1, j)=A(j, j+1)=0)$, then $\operatorname{ALPHAI}(j)=0$.

BETA (output) DOUBLE PRECISION array, dimension (N) BETA $(1: \mathbb{N})$ will be set to the (real) diagonal elements of $B$ that would result from reducing (A, B) to generalized Schur form and then further reducing them both to triangular form using unitary transformations s.t. the diagonal of $B$ is non-negative real. Thus, if $A(j, j)$ is a $1 \times 1$ block (i.e., $A(j+1, j)=A(j, j+1)=0)$, then BETA $(j)=B(j, j)$.

(Note that BETA(1:N) will always be non-negative real, and no BETAI is necessary.)

(input/output) DOUBLE PRECISION array, dimension (LDQ,N) (input/output) DOUBLE PRECISION array, dimension (LDZ,N) On entry, if WANTQ and/or WANTZ, the matrices $Q$ and $Z$ of generalized Schur vectors.

On exit, if WANTQ and/or WANTZ, $Q$ and/or $Z$ have been postmultiplied by the orthogonal transformation matrices which reorder (A, B); The leading $M$ columns of $Q$ and $Z$ form orthonormal bases for the specified pair of left and right eigenspaces (deflating subspaces). If WANTQ = .FALSE., $Q$ is not referenced. If WANTZ $=$.FALSE., $Z$ is not referenced.

(input) INTEGER

The leading dimension of the array $Q$.

LDQ $>=1$; and if WANTQ, LDQ $>=N$.

\section{LDZ (input) INTEGER}

The leading dimension of the array $Z$.

LDZ $>=1$; and if WANTZ, LDZ $>=\mathbb{N}$.

(output) INTEGER

The dimension of the specified pair of left and right eigenspaces (deflating subspaces). $0<=M<=N$.

PL, PR (output) DOUBLE PRECISION

If $\mathrm{IJOB}=1,4$ or $5, \mathrm{PL}, \mathrm{PR}$ are lower bounds on the reciprocal

of the norm of "projections" onto left and right eigenspaces, respectively, w.r.t. the selected cluster. $0<\mathrm{PL}, \mathrm{PR}<=1$. See also further details and references [2-3].

If $\mathrm{M}=0$ or $\mathrm{M}=\mathrm{N}, \mathrm{PL}=\mathrm{PR}=1$.

If $\mathrm{IJOB}=0,2$ or 3 , PL and PR are not referenced.

DIF (output) DOUBLE PRECISION array, dimension (2). If IJOB $>=2, \operatorname{DIF}(1: 2)$ store the estimates of Difu and Difl, respectively. If IJOB $=2$ or $4, \operatorname{DIF}(1: 2)$ are $F$-norm-based upper bounds on Difu and Difl, respectively. If $\mathrm{IJOB}=3$ or $5, \operatorname{DIF}(1: 2)$ are 1 -norm- 


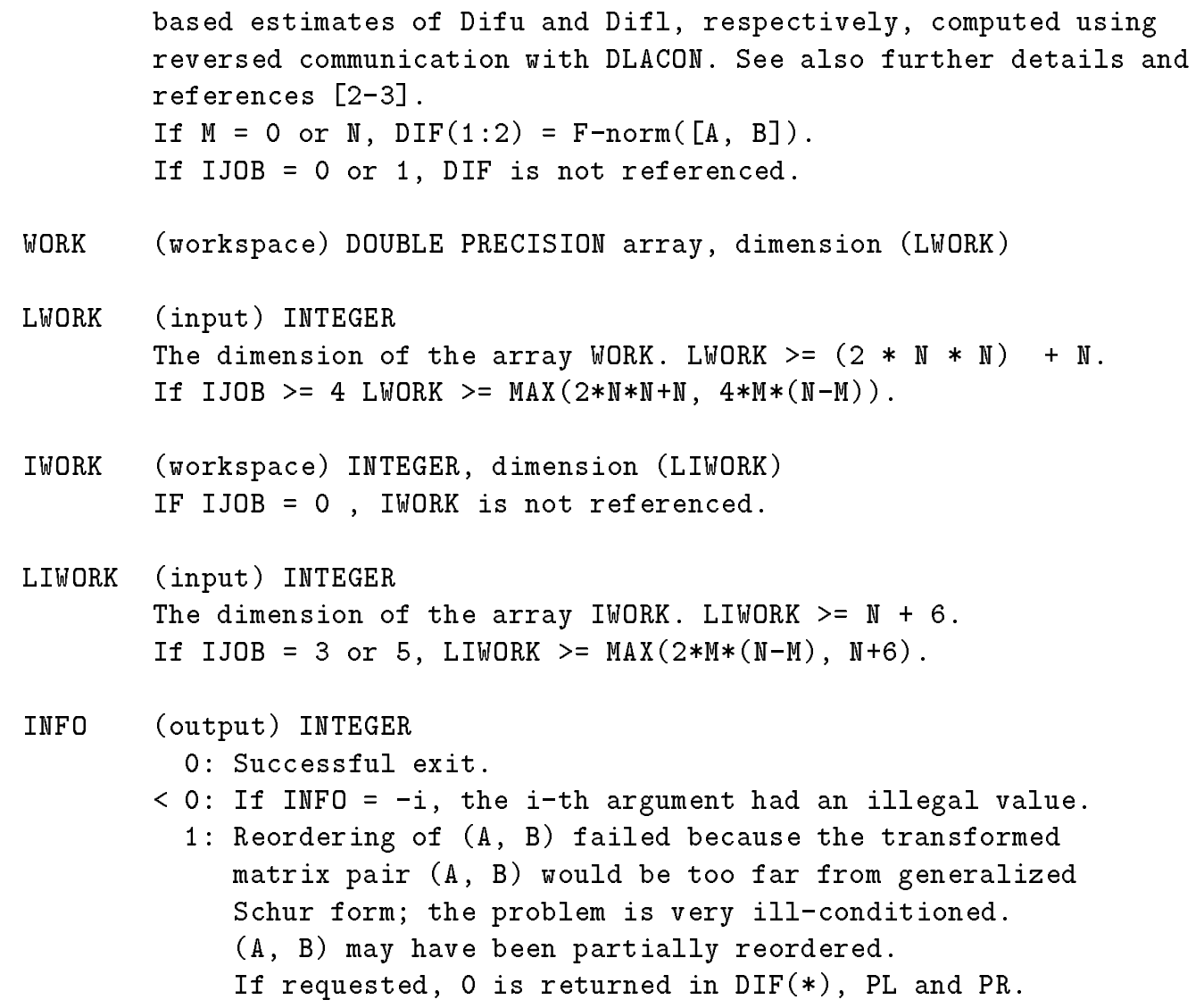

\section{Calling sequence DTGSNA}

Here we display the parameter list and the leading comment lines of the double precision routine DTGSNA.

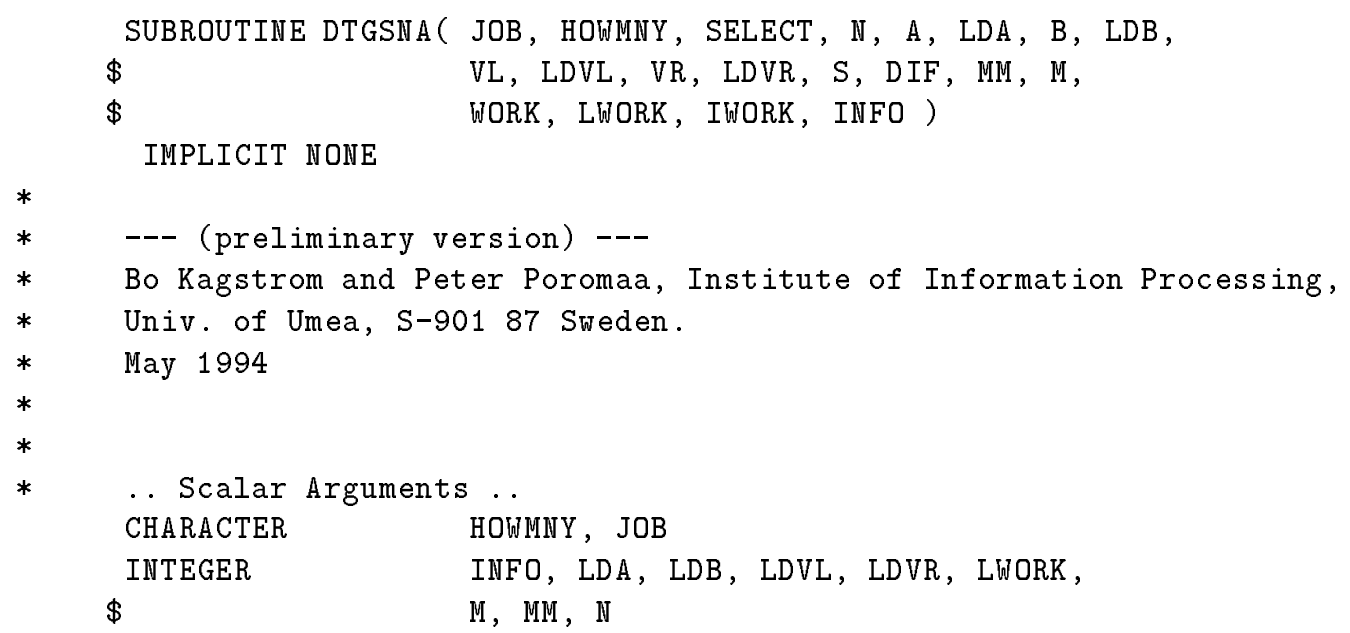




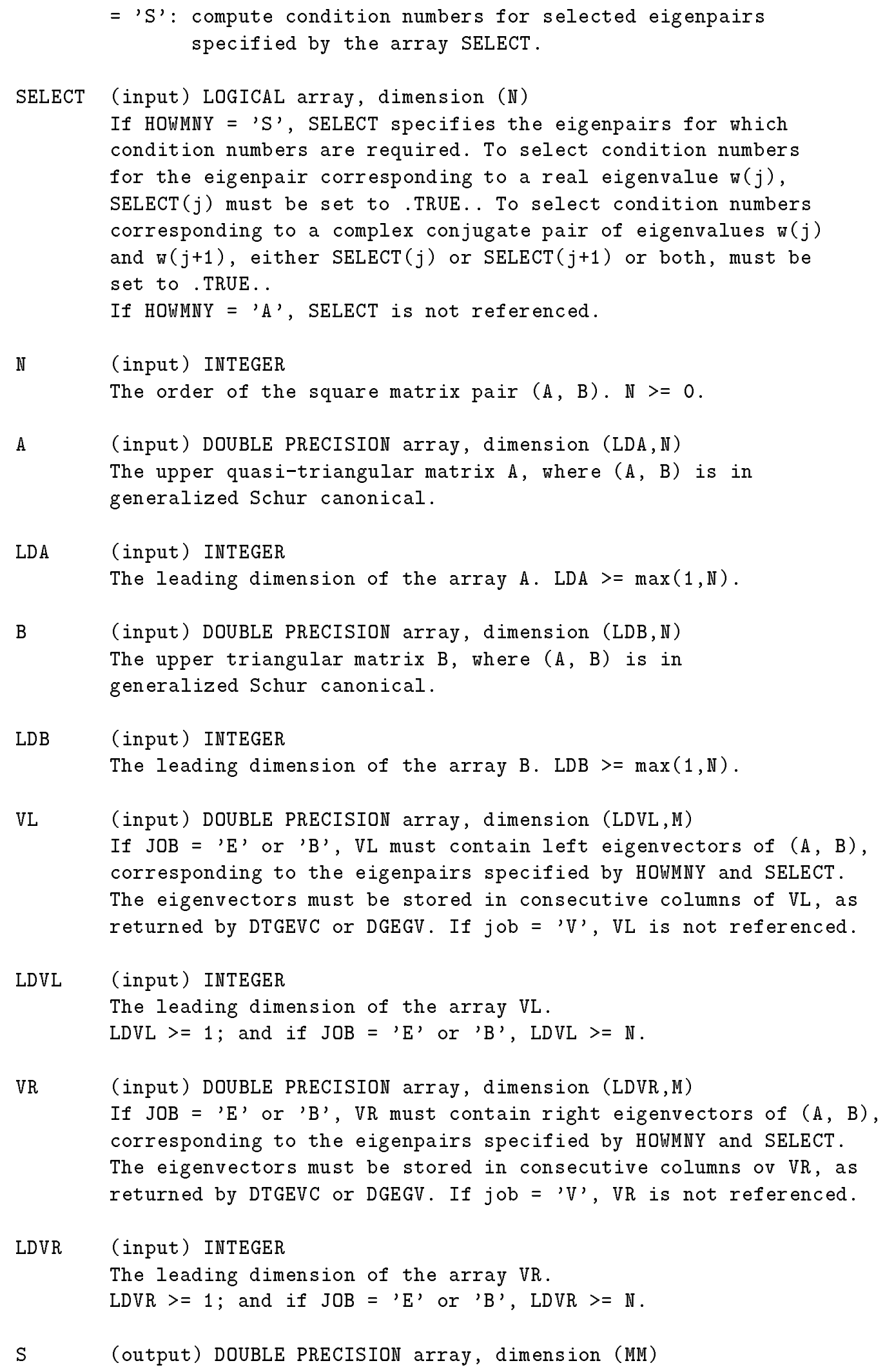




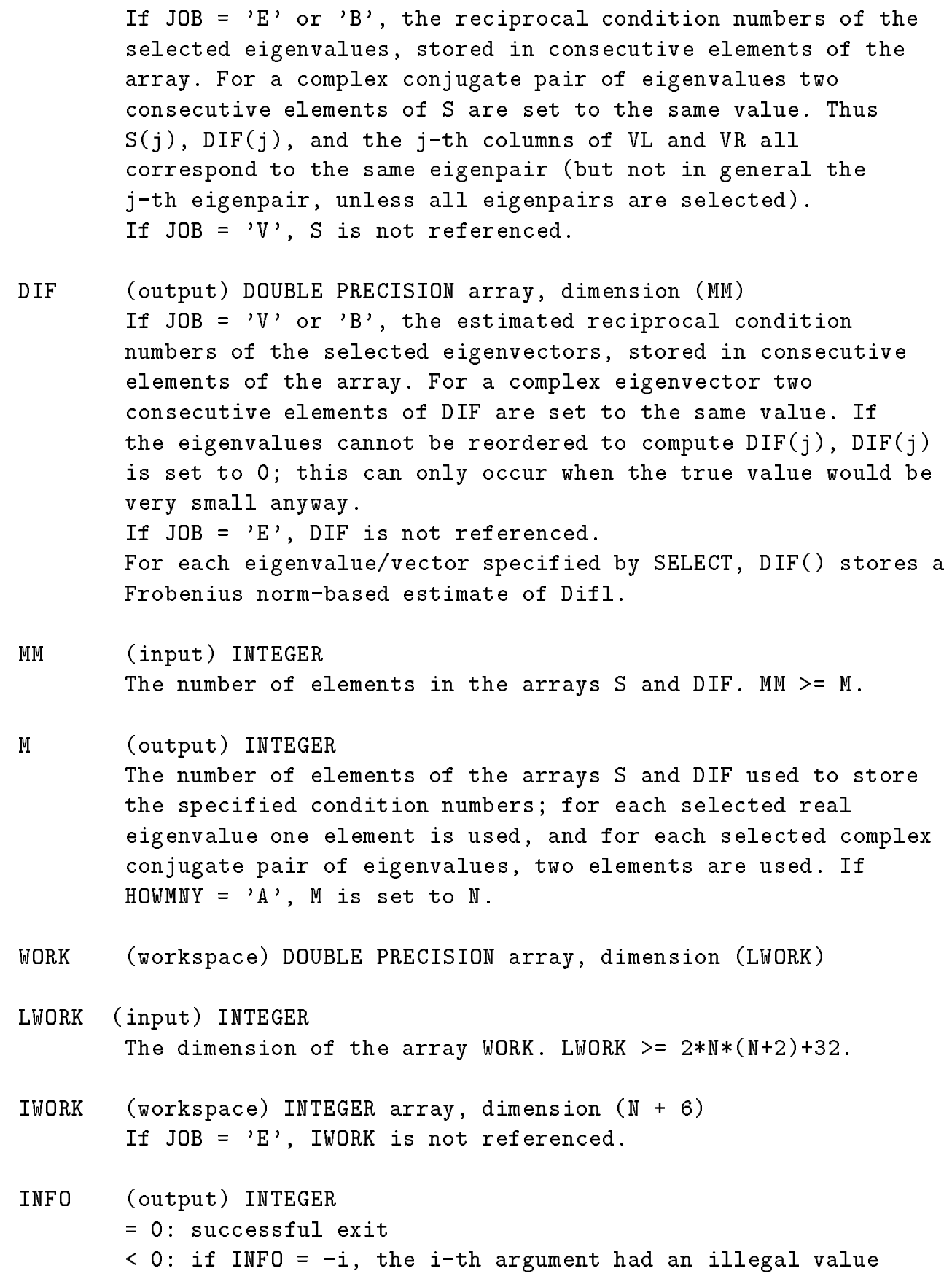

\section{E Calling sequence DGSRBB}

Here we display the parameter list and the leading comment lines of the double precision routine DGSRBB

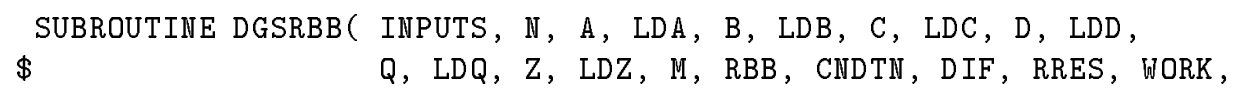




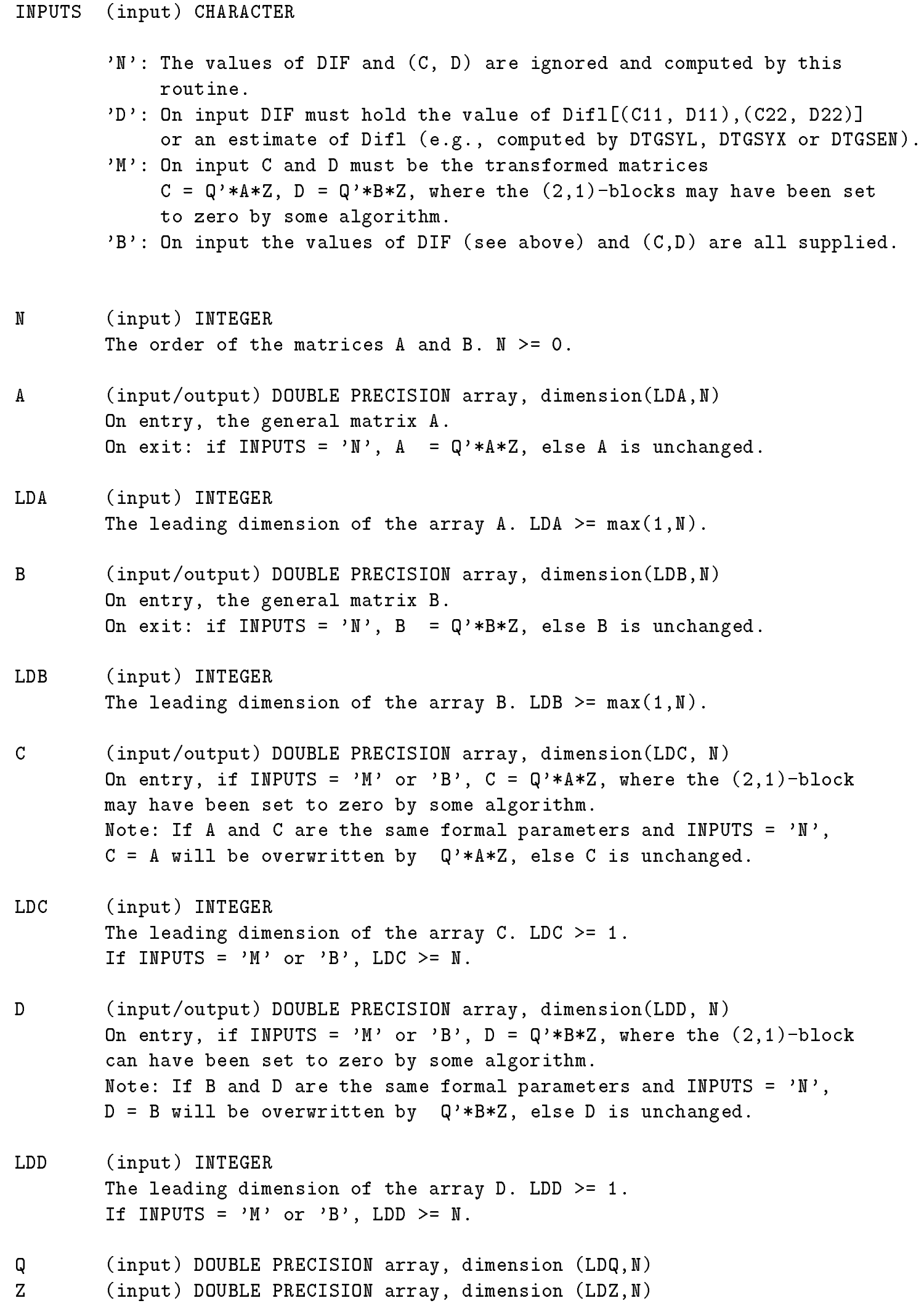




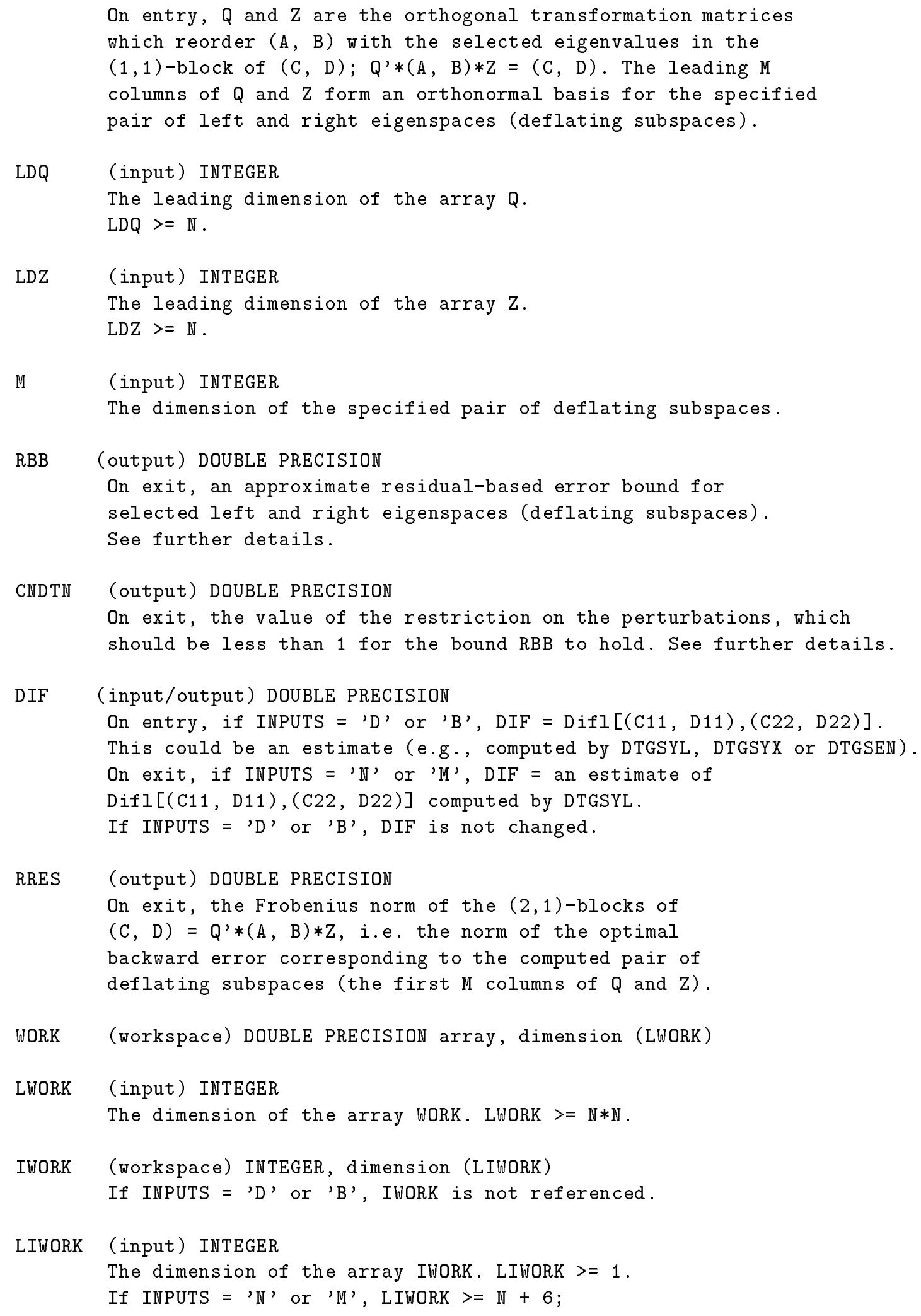



* INFO (output) INTEGER
* $\quad 0$ : Successful exit
* <0: If INFO $=-i$, the $i$-th argument had an illegal value
* $\quad 1$ : CNDTN $>1$, see further details.

* 